University of San Diego

Digital USD

2011-01-27

\title{
A Retrospective Study of Substance Use and Mental Health Disorders in a Sample of Urban American Indian and Alaska Natives
}

Kathryn Aimee Fuller PhD

University of San Diego

Follow this and additional works at: https://digital.sandiego.edu/dissertations

Part of the Nursing Commons

\section{Digital USD Citation}

Fuller, Kathryn Aimee PhD, "A Retrospective Study of Substance Use and Mental Health Disorders in a Sample of Urban American Indian and Alaska Natives" (2011). Dissertations. 403.

https://digital.sandiego.edu/dissertations/403

This Dissertation: Open Access is brought to you for free and open access by the Theses and Dissertations at Digital USD. It has been accepted for inclusion in Dissertations by an authorized administrator of Digital USD. For more information, please contact digital@sandiego.edu. 


\author{
UNIVERSITY OF SAN DIEGO \\ Hahn School of Nursing and Health Science \\ DOCTOR OF PHILOSOPHY IN NURSING
}

A Retrospective Study of Substance Use and Mental Health Disorders in a Sample of Urban American Indian and Alaska Natives

By

Kathryn Aimee Fuller

A dissertation presented to the

FACULTY OF THE HAHN SCHOOL OF NURSING AND HEALTH SCIENCE UNIVERSITY OF SAN DIEGO

In partial fulfillment of the requirements

for the degree

DOCTOR OF PHILOSOPHY IN NURSING

January 27, 2011

Dissertation Committee

Cynthia D. Connelly, PhD, RN, FAAN, Chairperson

Jane M. Georges, PhD, RN

Andrea L. Hazen, PhD 
(C) Copyright by Kathryn Aimee Fuller, 2011

All Rights Reserved 


\begin{abstract}
This retrospective study examined the prevalence of mental health disorders, cooccurring disorders (COD), and alcohol, tobacco, and other drug use (ATOD) among a sample of urban-dwelling adult American Indian and Alaska Natives (AI/ANs) seeking behavioral health services from one metropolitan Indian clinic in Southwestern United
\end{abstract} States.

A descriptive quantitative design employed retrospective data from AI/AN subjects $(N=123)$ verified as tribally enrolled and receiving outpatient behavioral health services. Chart abstraction included patient demographics, substance use and mental health diagnoses, and ATOD scores from the Patient Health Questionarre-9 (PHQ-9) and the Addiction Severity Index-NAV (ASI-NAV). The $t$-test compared gender differences and age at first use of commonly abused substances. Chi-Square $\left(\chi^{2}\right)$ determined proportional differences among gender, mental health, ATOD, and COD. Logistic regression examined contributory factors increasing the likelihood of a mental health or substance use disorder (SUD).

This urban adult AVAN sample was evenly distributed by gender (64 males, 59 females), with a mean age of 38.94 years $(S D=11.01)$. Prevalence rate for current smokers was $44 \%$, similar to nationwide data. Findings included diagnoses of mental health disorders $(79 \%)$, substance abuse disorders (76\%), and co-occurring disorders (55\%). For those subjects who completed the PHQ-9 $(n=46)$, the prevalence rate for depression was $61 \%$; the prevalence of depression among the 122 subjects with $I C D-9$ depression codes was $65 \%$. ASI-NAV composite scores (CS) in subject charts $(n=43)$ demonstrated positive, statistically significant correlations between the psychosocial CS 
of alcohol use, legal ( $r=.35)$, family $(r=.37)$, and psychiatry $(r=.32)$; drug use, legal $(r=.32)$, and family $(r=.36)$. Legal also positively correlated with medical $(r=.38)$, and family with psychiatry $(r=.38)$. Logistic regression identified one predictor as statistically reliable in mental health (housing) and two predictors in distinguishing status of substance abuse (unemployment and education). Subjects with higher levels of education were less likely to have a diagnosed SUD.

Health care providers rely on accurate data. Discerning the prevalence of mental health and substance use disorders when treating a growing native population ensures that culturally appropriate treatments are focused on the reduction of health disparities. 


\section{DEDICATION}

I would like to dedicate this dissertation by honoring all of my Indian elders and those who are no longer with us but always present in spirit like my youngest sister Julie. It was my grandmother, great-aunt, and my mom who taught me to remember that I am a Delaware or Lenape, and to make my family proud through my actions. I am equally grateful for my patient husband, Christian. I thank the Creator for allowing me the opportunity to love, nurture, and encourage my daughters, Kate and Bella, in achieving their own dreams.

I have been taught by some of the best nurses, including my mom who gave me the pearls of wisdom not found in any book. I gained the most valuable lessons in nursing from listening to the voice of other wise nurses while observing their compassionate nature and incorporating elements of their professionalism into my own nursing practice. 


\section{ACKNOWLEDGEMENTS}

First, I would like to thank my exceptional dissertation chair, Cynthia Connelly, Ph.D., R.N., F.A.A.N, Director of Nursing Research at the University of San Diego (USD) for her tireless efforts in supporting me through this academic endeavor. Under the direction of Dr. Connelly, I received invaluable guidance and advice that were essential elements to my success throughout the entire doctoral nursing program.

Indebted to her, I hope to honor Dr. Connelly's commitment to nursing through my future writings.

I would also like to thank my other committee members, Jane Georges, Ph.D., R.N., for her expertise in theoretical nursing theories and assistance in navigating through the Institutional Review Board (IRB) process. I am also thankful to committee member Andrea Hazen, Ph.D., for her suggestions, time, and knowledge regarding the improvement of my dissertation writings. All of my gratitude and special thanks to Patricia Roth, Ed.D., R.N., Director of the Doctor of Philosophy in Nursing program at USD. Dr. Roth encouraged me to continue writing when all seemed lost on paper. A special thanks to my mentor Donna Agan, Ed.D., for her ability to explain complex statistical analyses and knowledge regarding publication. My sincere appreciation to Sally Brosz Hardin, Ph.D, R.N., F.A.A.N., Dean of USD, Ann Mayo, DNSc., APRN, and Susan Instone, DNSc., R.N., for creating a supportive learning environment. I would also like to thank Susie Merrill, Administrative Assistant, and Cathleen Mumper, M.A., Director of Student Services and Admissions at USD for their efforts in meeting the needs of each student. 
It would have not been possible to complete this doctoral program without the support and funding provided by the Substance Abuse and Mental Health Services Administration (SAMHSA) Minority Fellowship Program (MFP), and the American Nurses Association (ANA). The staff of the MFP/ANA works tirelessly to support the fellows' academic successes. I was blessed with an opportunity to be mentored throughout my doctoral program by Faye Gary, Ed.D., R.N., F.A.A.N., Director of the ANA/MFP. Dr. Gary is invested in the success of each MFP fellow. I would also like to thank Janet Jackson, ANA/MFP Project Director. A special thanks to Hossein Yarandi, $\mathrm{Ph} . \mathrm{D}$. who patiently taught the fellows statistics and was always available for consultation. I am grateful to be part of the ANA/MFP fellowship. As a result, I have established invaluable life-long relationships and professional connections that will contribute to my future success as a nurse scientist. 


\section{TABLE OF CONTENTS}

List of Tables xii

List of Figures ............................................................................................. xii

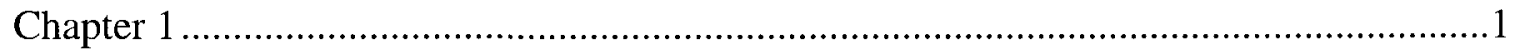

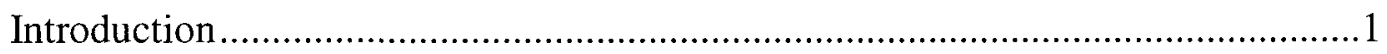

American Indian and Alaska Native (AI/AN) Demographics ............................

Health Status and the Invisible Tribe .......................................................6

The Effects of Misclassification of Race …....................................................

Mental Health, Substance Abuse, and Substance Misuse.................................7

Prevalence of Depression in American Indians and Alaska Natives (AI/ANs)......9

Alcohol Use Disorders among AI/ANs and the General Population.....................10

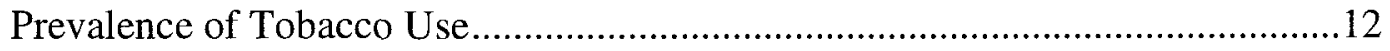

Statement of the Problem ....................................................................... 14

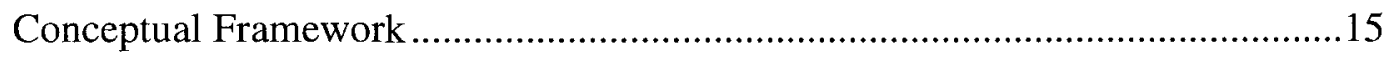

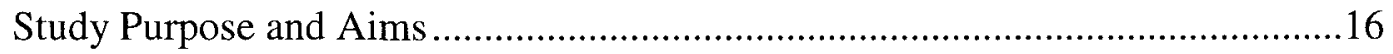

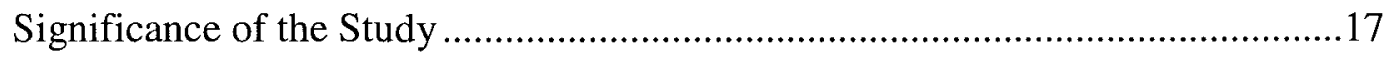

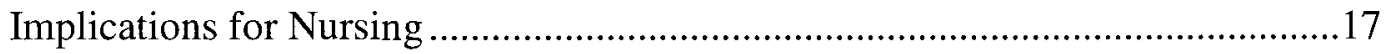

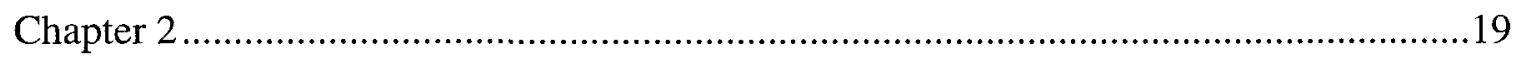

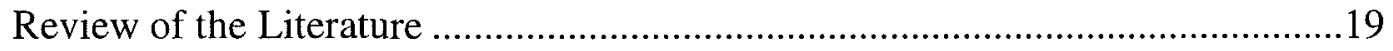

Co-occurring Disorders and Comorbidity Defined..................................... 19

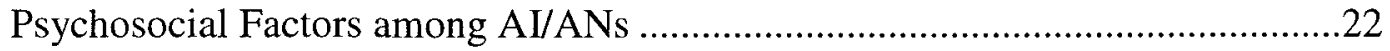

AI/AN Risk and Protective Factors .........................................................22

Historical Prevalence of Alcohol and Other Drug Use.....................................23 
Alcohol Misuse and Alcohol Use Disorders

Co-occurring Disorders among U.S. Population and AI/ANs ..........................28

Historical Trauma and Possible Causes for Substance Abuse Disorders ...............34

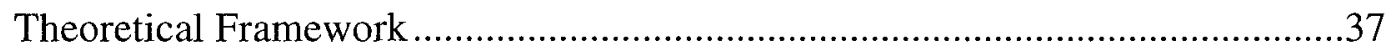

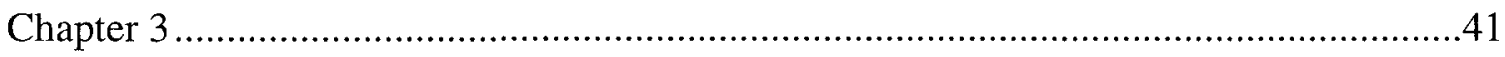

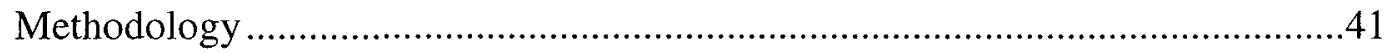

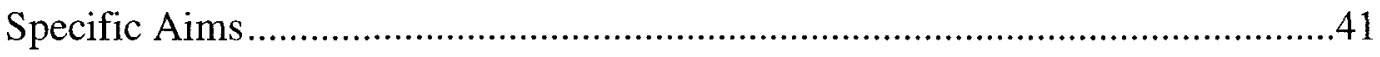

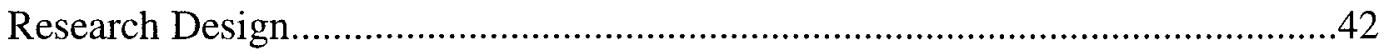

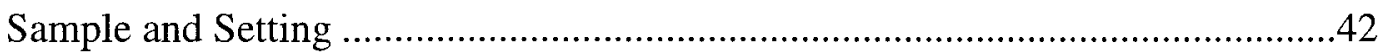

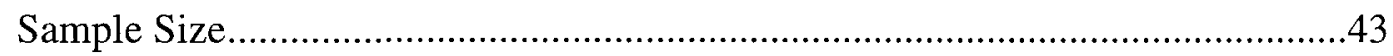

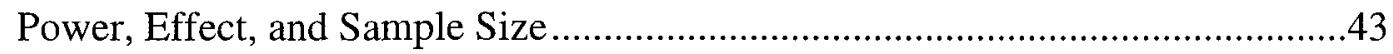

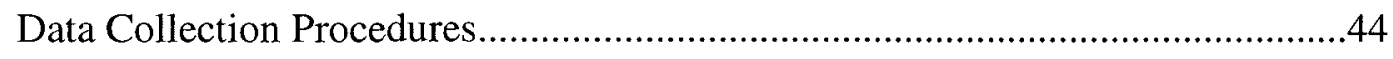

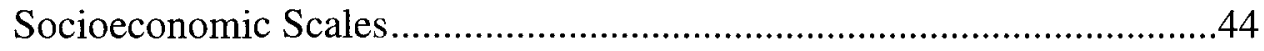

Brief Assessment ................................................................... 44

Addiction Severity Index-Native American Version (ASI-NAV)............45

Patient Health Questionnaire-9 (PHQ-9) .........................................46

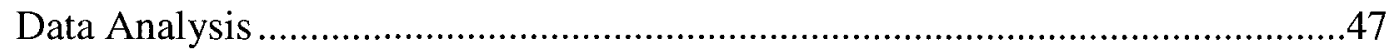

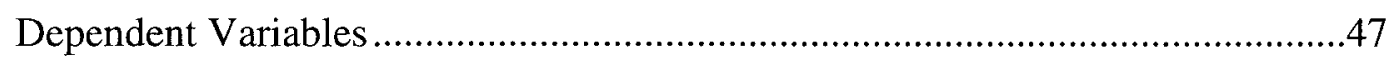

Independent Variables ................................................................... 47

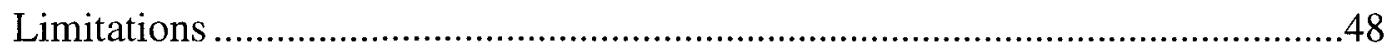

Human Subjects Protection ................................................................. 48

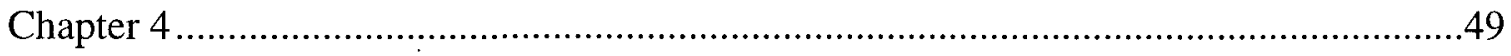

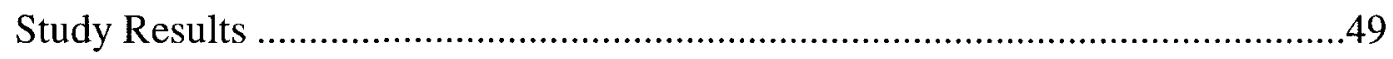


Characteristics of the Sample.

Descriptive Characteristics

Gender. .58

Smoking (Tobacco Use)

Thirty Day and Reported Years of Substance Use

Thirty Day and Lifetime Prevalence of Suicide and Violence by Gender .63

Interviewer's Rating of Need for Treatment by ASI-NAV CS 67

Patient's Rating of Need for Treatment by ASI-NAV CS. .68

Correlation among ASI-NAV Composite Scores. 69

Spirituality .70

Cross Tabulation 71

Mental Health and Exploratory Variables 72

Substance Use Disorders and Exploratory Variables .74

Logistic Regression for Mental Health Disorders ......................................... 78

Logistic Regression and Substance Abuse Disorders ....................................8

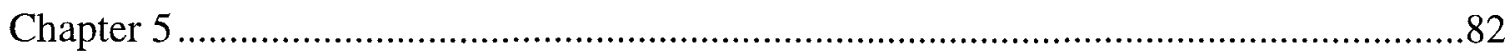

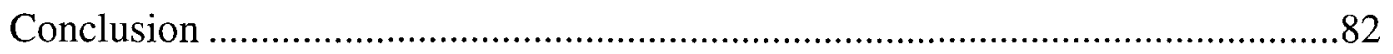

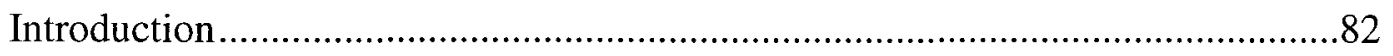

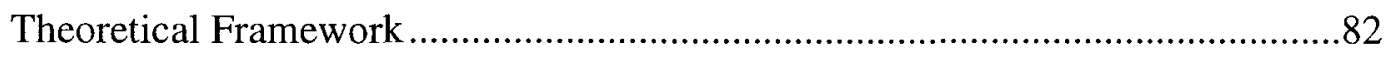

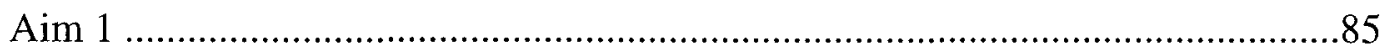

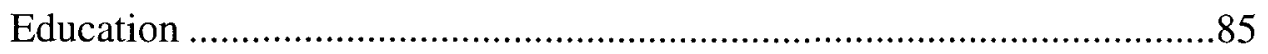

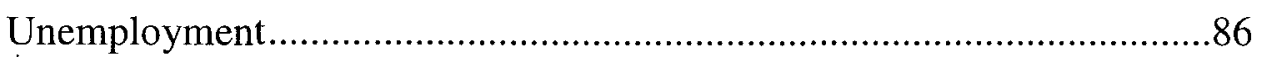

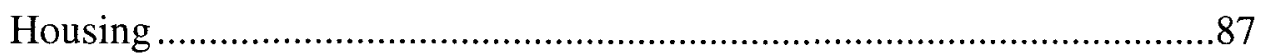


Aim 2

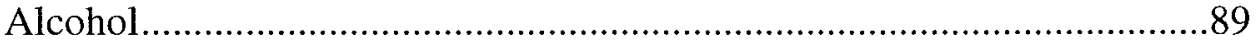

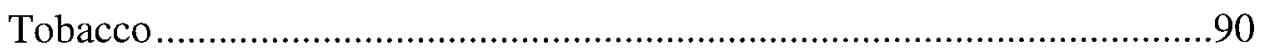

Other Illicit Drug Use ...........................................................................

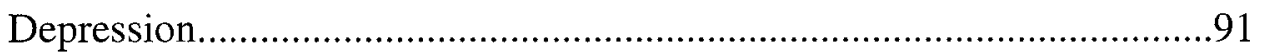

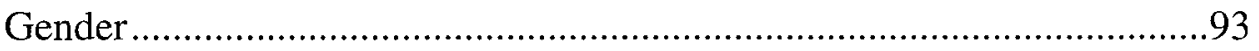

30 Day and Reported Years of Substance Use ..........................................94

30 Day and Lifetime Reports of Violence …….........................................94

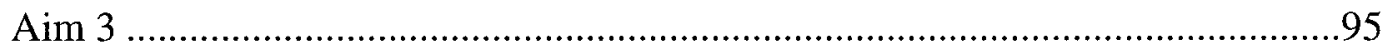

Interviewer's Rating of Need for Treatment by ASI-NAV CS .................95

Patient's Rating of Need for Treatment by ASI-NAV CS.........................96

Correlation of ASI-NAV Composite Scores...............................................97

Correlation of ASI-NAV Scores and Spirituality .......................................98

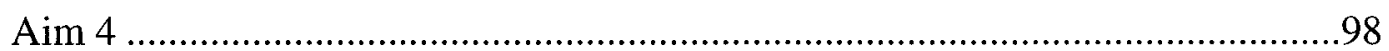

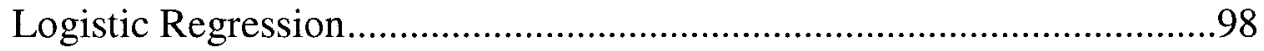

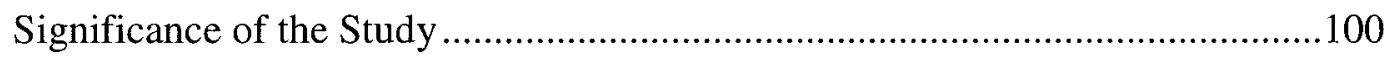

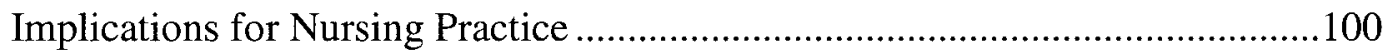

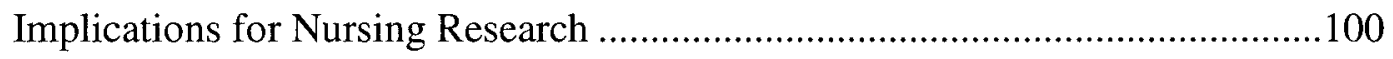

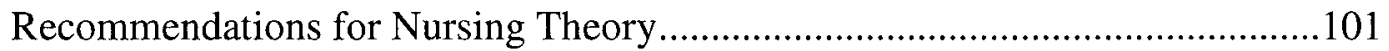

Recommendations for Nursing Education ........................................................102

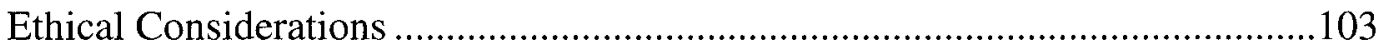

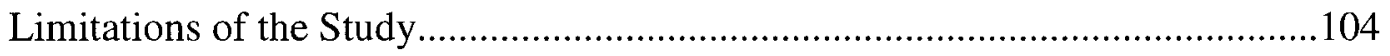

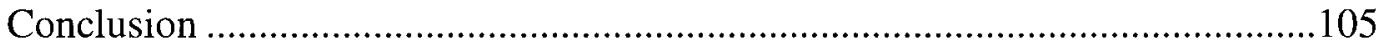




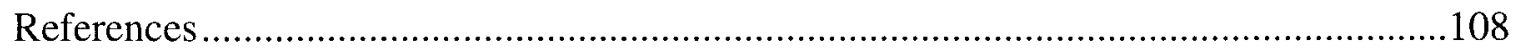

Appendix A: USD Institutional Review Board Approval .............................................140

Appendix B: Department of Health \& Human Services IHS NIRB Approval.................141 


\section{LIST OF TABLES}

Table 1 Descriptive Characteristics .51

Table 2 Highest Frequency of Reported ICD-9 Codes 53

Table 3 Sample Characteristics by Reported Incidence of Substance Abuse and Gender

Table 4 PHQ-9 Results 55

Table 5 PHQ-9 Severity Scores .56

Table 6 Diagnosis of Depression: PHQ-9 verses Clinical Documentation .... .57

Table 7 Chart Documentation of Age at First Use by Gender. .59

Table 8 Mental Health Disorders and Substance Abuse by Gender .60

Table 9 Distribution of Gender by Smoking Status .62

Table 10 Substance Abuse as Reported on ASI-NAV .63

Table 11 Thirty Day and Lifetime Reports of Suicide and Violence .65

Table 12 Pearson $r$ Correlations among the ASI-NAV Composite Scores...... .70

Table 13 Spirituality Subscale Question of the ASI-NAV .71

Table 14 Cross Tabulation for Mental Health Diagnosis and Housing. .73

Table 15 Cross Tabulation for Mental Health Diagnosis and Employment .74

Table 16 Cross Tabulation for Substance Use Disorders and Housing..... .75

Table 17 Cross Tabulation for Substance Use Disorders and Employment .76

Table 18 Cross Tabulation for Substance Use Disorders and Education .77

Table 19 Logistic Regression Coefficients Related to Mental Health Disorders .79

Table 20 Logistic Regression Coefficients with SUD as the DV 


\section{LIST OF FIGURES}

Figure 1 Broad American Indian Worldview Model _...................................................39

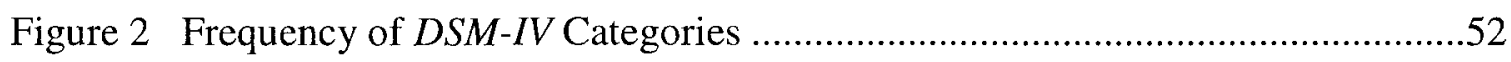

Figure 3 Interviewer Severity Ratings of the Subjects Need for Treatment on

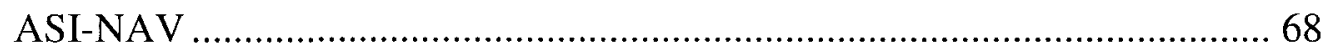

Figure 4 Patient's Rating of Severity and Need for Counseling Services .......................69 


\section{Chapter 1}

\section{Introduction}

Eliminating health disparities among American Indian and Alaska Native (AI/AN) people remains a public health priority. As described in Healthy People 2010, "the goal is to raise the health status of AI/AN people to the highest level possible" (U.S. Department of Health \& Human Services [USDHHS], 2006). To build and expand upon Healthy People 2010 and the Institute of Medicine (IOM) report on Unequal treatment: Confronting racial and ethnic disparities in health care, the Agency for Health Care Research and Quality (AHRQ), annually conducts the broadest examination of disparities in health. The findings are disseminated in the National Healthcare Disparities Report (NHDR) in an attempt to identify which disparities in health care contribute to overall disparities in health. NHDR concentrates on the national view of health care disparities; as pointed out in their 2006 report, many causes of disparities and priorities addressing them vary across the country. NHDR argues eliminating disparities in health care is a logical method for eliminating associated disparities in health and focused community based projects supported by local data are necessary to successfully address these disparities (Agency for Health Care Research \& Quality [AHRQ], 2008; Institute of Medicine [IOM], 2002). 
Health care for mental illness, substance use disorders, and general illnesses must be viewed as equally paramount when addressing health care disparities, since chronic illness is the leading cause of disability, illness, and death in the United States (IOM, 2006). Research indicates that patients who present with a psychiatric and or substance use disorders are often at increased risk for the other problem (Reiger et al., 1990). A patient's ability in accessing appropriate behavioral health services becomes complicated when faced with homelessness, incarceration, or overall poor health. In response to the growing need to address mental health and substance use disorders, a national objective to address mental health disparities has been outlined by Healthy People 2010, 18-10 which is, "to increase the proportion of persons with co-occurring substance abuse and mental disorders who receive treatment for both disorders' (USDHHS, 2006). The previous mental health and mental disorder (MHMD) objective from Health People 2010 was retained but modified in Healthy People 2020, MHMD HP2020-14 as stated to, 'increase the proportion of person's with co-occurring substance abuse and mental disorders who receive treatment for both disorders' (USDHHS, 2009).

There are few published studies that have examined the mental health needs of urban American Indian and Alaska Natives (AI/ANs) struggling with comorbid disorders, while there are many studies revealing that AI/ANs living on designated reservations have higher rates of reported violence, post-traumatic stress disorder (PTSD), mental disorders, alcohol, and substance misuse than the general population (Beals et al., 2002; Manson, Beals, Klein, \& Croy, 2005). These mental conditions combined with physical problems such as cardiovascular disease, diabetes, unintentional injuries, trauma, tobacco-related illnesses, and specific infectious diseases contribute greatly to the high 
rates of health disparities in the literature, which impact the AV/AN population (Castor et al., 2005; Rhoades, 2006; Rhoades, Welty, Wang, Devereux, \& Fabsitz, 2007).

\section{American Indian and Alaska Native (AI/AN) Demographics}

AV/ANs are grouped together by race throughout the literature as they share original common origins from North and South America while maintaining community ties or tribal affiliations (Centers for Disease Control \& Prevention [CDC], 2002a). As of July 2009 , total population estimates for the United States were $307,006,550$ persons (U.S. Census Bureau, 2009). Out of total population estimates, 4.3 million persons identify themselves as American Indian or Alaska Native, comprising $1.5 \%$ of the total population (U.S. Census Bureau, 2006). During 1990-2000, the AI/AN population increased $26 \%$, compared with $13 \%$ for the total U.S. population. Of all racial/ethnic populations, $\mathrm{AI} / \mathrm{ANs}$ have the highest poverty rates $(26 \%)$ that is twice the national rate, predisposing AV/ANs to greater health disparities (CDC, 2003a). In 2007, Healthy People 2010 identified that AV/ANs have a larger proportion of health disparities in greater than or equal to $100 \%$ of categories as defined when compared to other ethnic populations (USDHHS, 2007).

Approximately 565 federally-recognized tribes reside in rural and urban areas of the United States. Governmental programs providing tribal infrastructure for eligible Indians are administered directly by Indian Health Service (IHS) and the U.S. Bureau of Indian Affairs (BIA) via grants that improve Indian communities (BIA, 2009, 2011). Regardless of location, Native descent from a federally recognized tribe is a defined term commonly used for Indian identity (National Urban Indian Family Coalition [NUIFC], 2007). It has been estimated that $60-67 \%$ of the total AI/AN population live in urban or 
metropolitan areas across the United States, which occurred through relocation efforts enacted by the federal government (CDC, 2002a; Urban Indian Health Commission [UIHC], 2007). AI/ANs residing in urban areas have different cultural experiences and unique health problems as compared to Indians living on designated reservations or in rural areas. Historically, the lack of adequate federal aid to facilitate the urbanization of Indians has been the result of failed government policies that place urban AI/ANs at greater risk for health disparities (UIHC, 2007).

Urban Indians often face barriers when attempting to access health care. In 2006, there were only 34 organizations that provided health care services for urban AI/ANs and less than $2 \%$ of Indian Health Service's $\$ 3$ billion annual budget was spent on urban Indian health care services (UIHC, 2007). For the 2009 fiscal year, 18 urban Indian health programs in the Southwest manage full service health programs on a budget of approximately $\$ 11$ million dollars (IHS, 2008a). California has one of the largest populations of $\mathrm{AI} / \mathrm{ANs}$ in the United States and yet only $10 \%$ of California Indians had access to general health care services (Seals, Burhansstipanov, Satter, Chia \& Gatchell 2006; UIHC, 2007). Mental health services are available to approximately 605,000 AI/ANs but only one in five reported access to this care in 2000 (National Alliance on Mental Illness, 2007; USDHHS, 2001).

Patients with co-morbid psychiatric diagnoses often increase the utilization and cost of health services compared to patients with a single use disorders (Hoff \& Rosenheck, 1999). The annual cost to insure persons with serious mental illness (SMI) who are enrolled in a health maintenance organization (HMO) is approximately $\$ 19,788$ per year (Shern, Jones, Chen, Jordan, Ramoni-Perazzi, \& Boothroyd, 2008). Only few 
AI/ANs qualify for Medicaid or Medicare as a single payer source for medical care if they are not located by an IHS facility that provides health care services (UIHC, 2007). In 2007, estimates for annual total Medicaid charges related to the treatment of bipolar or comorbid conditions was $\$ 22,110$ and increase about $4 \%$ per year, thereafter (Guo, Keck, Li, \& Patel, 2007).

Obtaining medication for psychiatric disorders is problematic since Medicaid and other managed care insurance companies restrict formularies or limit access to newer and more appropriate psychotropics, which has a detrimental effect on behavioral health treatment plans (Donohue \& Frank, 2007). Urban Indian health programs provide low cost medications but have fewer resources to access branded pharmaceuticals due to monetary budget constraints. AI/ANs with co-occurring disorders (COD) may find it difficult to maintain their sobriety since there are few outpatient programs providing posttreatment support and often return to the communities that promoted their substance misuse (Young, Joe, Hassin, \& St. Clair, 2001). Struggling to identify the single source of fragmentation in substance abuse and mental health care services for urban AI/ANs is difficult to delineate.

The majority of what we know about AI/AN health and illness is based upon the limited research conducted with particular tribes residing on designated reservations. Alcohol use disorders (AUD) have been widely studied in AI/AN communities and often overshadows other illicit drug use within the population (Beals, Manson, Mitchell, Spicer \& AL-SUPERPFP, 2003; May \& Gossage, 2001; Whitesell, Beals, Mitchell, Novins, Spicer, O'Connell, et al., 2007). In general, there have been few studies addressing 
health and illness in the urban AI/AN population. Notably, "urban Indians" are referred to as "the invisible tribe" (UIHC, 2007).

\section{Health Status and the Invisible Tribe}

In 2008, the Urban Indian Health Institute (UIHI) reported findings from the Behavioral Risk Factor Surveillance System (BFRSS) that described urban AI/AN health as worse than the rest of the population. Moreover, only $44 \%$ of AI/ANs reported having "excellent or very good health" when compared to $56 \%$ of the rest of the population (UIHI, 2008). Current data show that some ethnic minorities, as well as low income families regardless of race or ethnicity tend to be in poorer health than other Americans (Kreiger, Williams, \& Moss, 1997). The interrelations between race, ethnicity, and socioeconomic status are complex. It is often difficult to tease out specific or individual contributors that increase the potential to experience poor health.

Assumptions are often made that most AV/ANs live on designated reservations and that the federal government covers their health care. Although there are two major statutes that have served as key pieces of legislation ensuring the government's trust responsibility for the provision of health care for enrolled AI/ANs authorized by 25 U.S.C. 13, Snyder Act, and the Reauthorization of the Health Care Improvement Act P.L. 94-437, as amended (USDHHS, 2007b). Today, nearly seven out of ten AI/ANs live near metropolitan areas, however there are no uniform federal policies mandating urban Indian health care (UIHC, 2007). Regardless of promises and Federal treaties made with federally recognized tribes, health care programs for AI/ANs are continuously funded at lower levels than other government health programs (Westmoreland \& Watson, 2006). 


\section{The Effects of Misclassification of Race}

The literature includes inaccurate morbidity and mortality reports that provide statistical health information on race and ethnicity. Historically, IHS and tribal registries have maintained data on morbidity \& mortality rates for rural AI/ANs but more than half of the population resides in metropolitan areas (Sugarman \& Grossman, 1996). The misclassification of race from death certificates leads to an under-reporting of health issues that impact AI/ANs, and yields unreliable health disparities data. Other studies concluded that racial misclassification results in the underestimation of the injury morbidity, certain disease states such as lung cancer and cardiovascular disease (Knight, Yankaskas, Fleg, \& Rao, 2008; Rhoades, 2005). Strong evidence suggests that errors in misclassification of race are generally one-way, and that $\mathrm{AI} / \mathrm{ANs}$ are usually classified as non-Indian (Puukka, Stehr-Green, \& Becker, 2005). One retrospective study found that $\mathrm{AI} / \mathrm{AN}$ persons with $100 \%$ Indian blood quantum were $4 \%$ likely to be misclassified in cases reviewed as compared to individuals with less than $25 \%$ blood quantum who were misclassified in 44\% of the examined cases (Stehr-Green, Bettles, \& Robertson, 2002). Estimating the epidemiological rates for mental health and substance abuse disorders among the AI/AN population may be difficult since there are no unified or single standardized linkage systems well studied for reliability and validity of published vital statistic data.

\section{Mental Health, Substance Abuse, and Substance Misuse}

There have been no large epidemiologic surveys reporting on the prevalence rates of co-occurring disorders among urban AI/ANs. A large portion of the data in the literature has been inferred from larger population studies or smaller studies that focus on 
particular tribes. In the general population, there have been five large epidemiological studies in the U.S. that are; the Epidemiologic Catchment Survey (ECA), National Comorbidity Survey (NCS), National Comorbidity Survey Replication (NCS-R), National Longitudinal Alcohol Epidemiologic Survey (NLAES), and the National Epidemiologic Survey on Alcohol and Related Conditions (NESARC). From these studies, the cumulative findings are suggestive of a positive correlation between alcohol and substance use disorders with only slight variances in published results (Stinson, Grant et al., 2006). With regard to prevalence rates of mental health, the NCS identified that approximately one-half of all persons in the U.S. will meet the diagnostic criteria for a diagnosed mental health disorder during their lifetime (Kessler, Berglund, Demler, Jin, Merikangas, \& Walters, 2005). Therefore, the likelihood of a clinician encountering an individual with one or more psychiatric comorbidities is increased. More than one-third of all visits to IHS facilities are related to substance use, mental health, or alcoholism and are frequently exacerbated by persistent health disparities that include problems related to psychosocial functioning or quality of life (IHS, 2008b).

The lack of empirical based evidence has highlighted that practicing clinicians for urban AI/ANs may have difficulty in assessing or prioritizing specific determinants of health requiring more clinical attention when addressing alcohol and drug related problems. A majority of the published studies on substance abuse and mental health disorders among AI/ANs analyze data from AI living on reservations, describe gender differences in substance abuse, or present findings that separate substance use from mental health disorders in published findings. There is increasing evidence suggesting that depression is more likely to occur with progressive substance abuse and that chronic 
substance abuse may be a contributing factor of the development of depressive disorders (Sinha \& Rounsaville, 2002).

\section{Prevalence of Depression in American Indians and Alaska Natives (AI/ANs)}

The American Psychiatric Association (APA; 1987) published the Statistical Manual for Mental Disorder III-Revised (DSM III-R) that applies criteria in determining psychiatric diagnoses. In a study by Beals and colleagues (2005), the DSM III-R was useful in establishing the prevalence of depression among two AI reservations, and later compared depression rates from the general population obtained from NCS findings. Overall, findings between the two AI reservations revealed higher rates of poverty, depressive/anxiety disorders, and PTSD. Lifetime rates of alcohol dependence were higher between both reservations but not among Southwest AI women in this study.

Lifetime major depressive disorders (MDD) were lower in both tribes as compared to the NCS findings (Beals et al., 2005). In contrast to the NCS findings, the National Survey on Drug Use and Health (NSDUH) reported past year prevalence rates for major depressive episodes (MDE) among subjects between the ages of 18 to 25 was highest among persons reporting origins of two or more races then followed by Native Hawaiian/Pacific Islanders and AI/ANs (USDHHS, 2007c).

The NESARC survey $(N=43,093)$ reported findings on depressive disorders and reported that AI had the highest lifetime prevalence of MDD (19.2\%) as compared with other ethnic groups (Hasin, Goodwin, Stinson, \& Grant, 2005). Among elderly AI, prevalence rates for depression were 32\% (Manson, 1989). A small study by Duran and colleagues (2004) identified that the lifetime prevalence for all documented mood disorders in primary care was $48 \%$ among a sample of 234 AI women. Additionally, 
$82 \%$ of the AI women who reported a mood disorder also indicated concurrent major depression.

Recent studies are suggestive of a strong association between mental health disorders and substance abuse (Jane-Llopis \& Matytsina, 2006). While the NESARC survey respondents indicated that $40.3 \%$ had experienced AUD, another study found that $72 \%$ of the adult psychiatric participants diagnosed with a depressive disorder also reported suicidal ideation (Roberts \& Yeager, 2004; Hasin, et al., 2005). Suicide research has documented the co-occurrence of suicidal ideation, MDD, and SUDs. The severity and chronicity of a depressive illness or recurrent MDD conveys the greatest suicide risk (Witte, Timmons, Smith, \& Joiner, 2008). Researchers have sought to identify specific risk factors pertaining to suicide, with fewer studies documenting the narrow range of behaviors or signs of near-term suicidal events (Rudd et al., 2006)

The highest rates for suicide in the AV/AN population are between the ages of 25 44 (CDC, 2003b). In 1997, IHS reported that AV/AN youth have the highest injury death rates from suicide, pedestrian related accidents, and motor vehicle crashes when compared to other races. American Indian youth engage in high risk behaviors more often that compromises their health as compared to other ethnic groups (NeumarkSztainer et al., 1996). Notably, health risk behaviors acquired in adolescence may lead to dysfunctional coping mechanisms in adulthood.

\section{Alcohol Use Disorders among AI/ANs and the General Population}

Substance abuse studies on alcohol abuse among AI/ANs have yielded conflicting results in published data. The CDC (2008a) analyzed data from death certificates between the years 2001-2005 and indicated that alcohol-attributable deaths (AADs) for 
all $\mathrm{AI} / \mathrm{ANs}$ were $11.7 \%$. An age-adjusted $\mathrm{AAD}$ rate was further applied in the study and was twice that when compared to the U.S. general population (36.3 vs. 29.9 years). The CDC then combined national data sources between the years of 2001-2005 to determine death rates and found that the annual average was 1,514 for AADs among AVANs. Out of that annual average, $771(50.9 \%)$ of AADs stemmed from acute causes, $743(49.1 \%)$ resulted from chronic causes with the leading cause of acute death from motor vehicle crashes. There were differences of AADs for gender and differences among tribal regions throughout the U.S. As for gender, AADs were higher among AI/AN men than women, and more common among the ages of 20-49 years old. According to the CDC (2008a) the highest numbers of AADs for AI/ANs were in the Northern Plains region, followed by the Southwest, and Pacific Coast areas with the fewest number of reports in Alaska.

Shore, Manson, and Buchwald (2002) conducted a large study among urban AI/ANs $(N=754)$ that screened for alcohol abuse while seeking primary care and found the frequency of lifetime alcohol abuse rate of $56 \%$ and a frequency of current alcohol abuse of $27 \%$. Typically, past year alcohol use is more prevalent among AI/ANs in the 18-26 year old age group (USDHHS, 2007c). This age group also represents a large proportion of $\mathrm{AI} / \mathrm{ANs}$ in the childbearing years.

Fetal Alcohol Syndrome (FAS) is a major public health concern in the AI/AN community as the prevalence of FAS in Alaska is 5.6 per 1,000 live births and among some $\mathrm{AI}$ tribes the rates are 1.5 to 2.5 per 1,000 live births. In the general population, FAS prevalence rates range from 0.2 to 1.0 (CDC, 2002b; May \& Gossage, 2001). Findings from several alcohol abuse studies have suggested high prevalence rates for 
alcohol misuse among AI/ANs but their findings remain unclear with varying results. Further exploration in the context of historical trauma may provide a clearer explanation of associated risk factors that may contribute to problem drinking and substance abuse within this population.

\section{Prevalence of Tobacco Use}

The NSDUH survey (USDHHS, 2007d) reported on, "Depression and the Initiation of Cigarette, Alcohol, and Other Drug Use among Young Adults," and concluded that young adults between the ages of $18-25$ years old, with a past year MDE were more likely to start smoking in the past year than those who did not report a MDE in the prior year. Several other studies found that smokers with other CODs have high prevalence rates of tobacco dependence closer to 80\% (Miller \& Gold, 1998; Shoptaw et al., 2002). Prevalence rates of tobacco use are similar among AV/ANs and approximately $41 \%$ are smokers (Grim, 2007). Several other larger studies identified that nearly one out of three AN women smoke cigarettes during pregnancy (Alaska Department of Health and Social Services, Tobacco Prevention and Control Program, 2007).

Literature reveals that AI/ANs initiate smoking at a younger age as compared to other ethnic groups, and that one out of two high school students are smokers (IHS, 2007). Tobacco use is a culturally acceptable and not considered "negative" by most AI (Grim, 2007). In Indian culture, traditional tobacco has had a role for 18,000 years in ceremony and is often used as medicine for healing, prayer offerings, and gift-giving. However, chronic tobacco use outside of ceremonial purposes lends itself to chronic illness. The rate of smoking for AI/AN women of reproductive age was (44.3\%) compared with White (29.4\%), African-American (23.4\%), Hispanic (16.4\%), and Asian- 
American and Pacific Islander (5.7\%); (CDC, 1998). In the AI/AN population, smoking tobacco increases the risk for sudden infant death syndrome (SIDS), low birth weight, stillbirth, preterm delivery, and infertility (CDC, 2009a). Kaplan and colleagues (1997) identified that tobacco use among Alaska Natives (Aleuts, Eskimos, Indians) was the leading cause of preventable mortality.

Historically, the tobacco industry has targeted the marketing of cigarettes to AI/ANs for sponsoring cultural events such as pow wows (CDC, 1998). The sale of tobacco products are not taxed when they are purchased on Indian reservations. Tailored preventative interventions may be difficult to develop on reservations where the sale of tobacco products stimulates tribal economy (Eichner et al., 2005). Tobacco use is an important behavioral risk factor considering that cardiovascular disease is the leading cause of death in AI/ANs (CDC, 2003b; Howard et al., 1999). One of the health consequences for $\mathrm{AI}$ women who smoke is a high rate of reported lung cancer, four times higher in the state of California (Bennett, 1992).

From a Health Risk Behavior Appraisal (HRBA), many individuals who engage in one health risk behavior tend to have several multiple health risk behaviors that need to be addressed and prioritized to reduce the greatest risk (Berry, Kim, Minde, \& Mok, 1987). Research has shown the correlation between nicotine dependence and MDD (National Institute of Health [NIH], 2005). Thus, a history of nicotine dependence and smoking cessation efforts often includes depression as a reported symptom of withdrawal (Li-Tzy \& Anthony, 1999). Some researchers identified gender differences in the effects of stressful life events on changes in smoking status. Women tend to continue smoking 
during stressful situations, whereas men were able to maintain abstinence from tobacco use (McKee, Maciejewski, Falba, \& Mazure, 2003).

\section{Statement of the Problem}

There are gaps in the existing literature regarding general health, mental health, substance abuse and other drug use disorders among urban dwelling AVANs. The association between depression as a chronic illness and suicide has been well established. Suicide has become a major public health concern among AI/ANs between 15-34 years old. Rates for suicide are 2.2 times higher than the national average, becoming the second leading cause of preventable death within this ethnic group (CDC, 2009b).

The interrelationships of health, stress, substance misuse, and coping have been documented in the literature but little attention is given to gender or specific cultural issues that impact the AI/AN population (Walters, Simoni, \& Evans-Campbell, 2002). Maintaining the ability to make healthy choices is dependent on the mental state of the individual (Grim, 2006). Tribal and cultural identity influences behaviors and attitudes of Native people towards health related issues (Lomay \& Hinkebein, 2006). The adverse consequences of governmental relocation efforts of AI/ANs to urban areas is compounded by socioeconomic factors that include low education levels, poverty, high unemployment rates, racial discrimination, eroding of family and cultural support (Aud, Fox , Kewal-Ramani, 2010;Grim, 2006).

As a result, unhealthy lifestyle choices that include unprotected sexual activity, substance abuse, acts of homicide, suicide, and other risk taking behaviors leads to high rates of reported unintentional injuries which often marks the beginning of chronic illness (Grim, 2006; Marsiglia, Nieri, \& Stiffman, 2006). Since mental health disorders are 
pervasive, the Healthy People 2020 MHMD HP-2020-14 objective was retained from previous years to 'increase the proportion of persons with co-occurring substance abuse and mental disorders who receive treatment for both disorders' (USDHHS, 2009). The development of appropriate preventative efforts or treatment is a priority; findings from this study may support existing interventions, or indicate the need for tailoring treatment to reduce the morbidity and mortality among urban dwelling AV/ANs with substance abuse and mental health disorders.

\section{Conceptual Framework}

There are conflicting opinions in the literature on how to measure and define ethnicity, culture, cultural, ethnic, and racial identity (House, Stiffman, \& Brown, 2006). Ethnicity and the term culture often intertwine, with a global definition referring to "clusters of people who have common cultural traits that they distinguish from those other people" (Smedley \& Smedley, 2005, p. 18). Ethnic or tribal identity is an ethnocentric belief that belonging to a clan is part of tribal traditionalism where people share stories, spiritual beliefs, and social customs. Unlike the Western world or Protestant ethic, the world view concept 'harmony ethos' is based on aboriginal belief of interpersonal avoidance and intra-group cooperation, respect for Mother Earth, Father Sky, and that all humans or living creatures are considered of equal importance in nature (French, 1995).

Indigenous group identity supports the human phenomenon necessary to maintain psychocultural boundary maintenance. As AI/ANs were forced to live within the dominant culture or urbanized, they were denied traditional enculturational practices without appropriate socialization; therefore, these individuals were more likely to 
experience psychocultural marginalism. The combining factors of personal disorganization and cultural alienation or 'anomie' of marginalized persons places them at greater risk for substance misuse as psychocultural boundaries breakdown

(French, 2004).

\section{Study Purpose and Aims}

The purpose of this descriptive study is to gain an understanding of prevalence rates for mental health disorders, alcohol, tobacco, and other drug use (ATOD), and cooccurring disorders (COD), among a sample of urban dwelling adult AI/ANs seeking behavioral health care at a urban AI/AN community clinic in Southwestern United States. Specifically, the aims of this study are:

Aim 1

To characterize a population of AI/ANs who are seeking mental health services at an urban Indian community clinic.

Aim 2

To examine the prevalence of mental health disorders, ATOD, and COD among AI/ANs who are seeking mental health services at an urban Indian community clinic.

$\operatorname{Aim} 3$

To identify factors that contribute to mental health disorders, ATOD, and COD among AI/ANs who are seeking mental health services at an urban Indian community clinic. Aim 4

To identify factors that increase the risk of substance use among AI/ANs who are seeking mental health services at an urban Indian community clinic. 


\section{Significance of Study}

Mental health disparities are complicated over the life span by factors of socioeconomic status, race/ethnicity, and geography. Certain mental health disorders exhibit different patterns of trajectory and prevalence among particular ethnic groups thus, learning how patterns of mental health disparities differ by disorder, and factors that contribute to a decline in functioning are imperative to closing the gap in treatment access while providing services to higher risk populations (National Institute of Mental Health [NIMH], 2010). The significance of the study lays in providing health care providers with accurate and useful data on the prevalence of mental health and substance use disorders among a growing native population with few available studies, and to inform evidenced based treatment programs that are culturally appropriate. A search of PubMed using the terms, urban "American Indian" and mental health, yielded merely 33 citations. In general, there are few descriptive or comparative studies addressing the prevalence of mental health disorders, COD, alcohol, and ATOD among urban AI/ANs; therefore, findings from this descriptive study may be useful in addressing specific mental health disparities that impact the urban AI/ANs.

\section{Implications for Nursing}

Healthy People 2020 retained the objective from Healthy People 2010, which is to 'increase the proportion of persons with co-occurring substance abuse and mental disorders who receive treatment for both disorders' (USDHHS, 2006a, 2009). A major step in addressing health care disparities is to increase access to mental health services. The passing of the Mental Health Parity and Addiction Equity Act of 2008 now ensures equal reimbursement for mental health care services in alignment with other medical or 
surgical billable services (USDHHS, 2010a). By 2025, studies are projecting a large physician shortage in rural and some urban areas (Kane et al., 2009).

Advance practice nurses are in the unique position of filling the provider gap and adding to the science of mental health. The development of culturally appropriate preventative efforts or treatment is a priority and therefore, findings from this study may support existing interventions, or indicate the need for tailoring treatments to reduce the morbidity and mortality among urban dwelling AV/ANs with substance abuse and mental health disorders. 


\section{Chapter 2}

\section{Review of the Literature}

\section{Co-occurring Disorders and Comorbidity Defined}

The literature on substance abuse and mental health disorders uses interchangeable terms when referring to dual diagnoses, co-occurring disorders and comorbidities. The term dual diagnosis has been widely referenced throughout earlier literature but the term co-occurring disorders is cited more often in newer published studies. In 2003, the Substance Abuse and Mental Health Services Administration (SAMHSA) developed the Co-Occurring Center for Excellence (COCE; USDHHS, 2006b) which serves as a national resource of information on substance abuse and mental health disorders but concluded the five year long project in March of 2009. The COCE website remains accessible to the public and has relevant information on COD.

The COCE (USDHHS, 2006b) defines and states "the term COD refers to cooccurring substance-related and mental health disorders" (p. 3). However, the COCE further delineates persons with COD by an individual-level definition, which indicates that a person met the criteria for more than one disorder and is not expressing multiple symptoms from a single use disorder. Although, the preferred term for use is the service definition, which indicates persons needing COD services. An illustrative example 
of the (service definition) suggests persons presenting in treatment are usually diagnosed with a single mental health disorder but frequently begin reporting other 'transitional' symptoms and acute signs of a co-occurring disorder from another psychiatric problem need COD services (USDHHS Center for Substance Abuse Treatment [CSAT], 2005). From a clinical perspective, the conceptual (service definition) of COD includes the 'prediagnosis, post-diagnosis, or unitary disorders presenting with symptoms of a cooccurring problem". Further use of the (service definition) of COD includes the provider's assessment in determining the level of risk to the patient, in determining available resources to meet short and long term goals, thereby improving program planning needs.

The term comorbidity is perplexing and is often defined differently in the medical, psychiatric, or social science bodies of literature. Mosby's Medical Dictionary (Mosby, 2008) defines comorbidity as "two or more coexisting medical conditions or disease processes are additional to an initial diagnosis". In review of the psychiatric literature, Stinson, Grant et al. (2006) refers to dual diagnoses as "the co-occurrence of a psychiatric and substance abuse disorder" while referring to comorbidity as "the cooccurrence or overlap of two or more psychiatric disorders" (p. 94).

Stinson, Grant and their colleagues (2006) redefine the term dual diagnoses by combining the definitions of homotypic comorbidity, which represents the "co-occurrence of disorders within a diagnostic grouping" such as nicotine dependence and alcohol use disorders, and refer to heterotypic comorbidity as "co-occurrence of two disorders from different diagnostic groupings" such as panic disorders and alcohol use disorders. These researchers identified five large epidemiologic studies world-wide that have focused on 
homotypic comorbidity of drug and alcohol use disorders in the world's general population. Three out of five epidemiologic studies occurred within the United States and were; the ECA, NCS, and NLAES (Grant \& Harford, 1995; Kessler et al., 1997; Reiger et al., 1990). The studies performed in the United States used subjects that were 18 years or older and assessed substance use disorders by using DSM-III criteria in the ECA, the $D S M I I I-R$ criteria for the NCS, and the $D S M-I V$ criteria for the NLAES (American Psychiatric Association [DSM-IV], 1994).

Outside of the United States, two of the five epidemiologic surveys completed were the 1990 Mental Health Supplement of the Ontario Canada Health Survey (MHSOHS) and the 1997 Australian National Survey of Mental Health and Well-Being (NSMHWB; Andrews, Hall, Teesson, \& Henderson, 1999; Ross, 1995). These two studies focused on probabilities of having simultaneous AUD and other SUDs, and vice versa but demonstrated notable differences in the methods employed to assess subjects for substance abuse by using the International statistical classification of diseases and related health problems, 10th revision (World Health Organization [ICD-10], 2007) and Composite International Diagnostic Interview (CIDI; Burns \& Teesson, 2002). Study subject included teenagers as young as 15 years old in the MHS-OHS. Conclusive findings among all of the studies identified with a few exceptions, the likelihood of having an AUD in persons with a drug use disorder (DUD; Stinson, Grant et al., 2006). Only the NSMHWB and NLAES surveys published homotypic comorbidity in a prior 12month period according to the diagnostic criteria cited in the DSM-IV (1994).

Limitations of prior studies include lack of specific data on treatment seeking for AUD or other drugs disorders with the exception of the NLAES. Later, the 2001-2002 
NESARC with 43,093 subjects addressed these limitations and categorically found that persons with comorbid and single drug disorders were more likely not female, of lower socioeconomic status, and never married. Findings from the alcohol only use category identified subject ages between 30-64 years old, White, and not of low socioeconomic status (Stinson, Grant et al., 2006). The NESARC was less concerned about associated factors between drug and alcohol use disorders. Therefore, it may be useful to study the psychosocial factors between alcohol and DUDs in the AI/AN population and the general population as well.

\section{Psychosocial Factors among AI/ANs}

The Merriam-Webster Online Dictionary (n.d.) defines psychosocial as "involving both psychological and social aspects" and "relating social conditions to mental health." In the broader terms, "psychosocial determinants of health" are related to the causal effects of "social structural factors" on outcomes of health that are modified or conditioned by social structures and individual-level contexts in which they exist (Martikainen, Bartley, \& Lahelma, 2002). Determinants of health are influenced by negative or positive effects that are frequently referred to as "risk or protective factors" (World Health Organization [WHO], 2004). Risk factors are variables that are considered 'negative' and produce socially undesirable outcomes. Protective factors are

considered 'positive' and decrease the likelihood of poor outcomes when exposed to risk (Jessor, Turbin, \& Costa, 1998).

\section{AI/AN Risk and Protective Factors}

Each tribe has their own traditions and cultural practices that define them as unique from one another. Due to this cultural diversity, risk, and protective factors may 
vary among tribal populations. Therefore, high reported prevalence rates of alcohol related morbidity or mortality, physical illness, mental health, and substance abuse disorders are indicators of underlying problems that warrant further exploration in assessing circumstances or influences that lead to the population's persistent health disparities, as cited throughout the literature. In a study conducted by Fagan (2006), findings indicated many variables that are considered risk factors may not be causal factors of ATOD, but are indicative of circumstances that occur prior to substance misuse and increase the likelihood for ATOD use. As the number of risk factors are increased and interact over time, an individual may be more susceptible to develop a SUD. There are no single theories that fully explain risk and protective factors in the AI/AN population but a plausible explanation can be provided in the context of historical events and perceived trauma.

\section{Historical Prevalence of Alcohol and Other Drug Use}

Until the end of the 19th century, fur traders, soldiers, miners, and cowboys frequently modeled high dose binge drinking and associated antisocial behavior that was observed by AI tribes who had little experience with alcohol problems (Winkler, 1968). According to Beauvais (1998) limited exposure to alcohol other than the use of fermented beverages or weak beer was used for ceremonial purposes prior to European colonization of the Americas; traders encouraged the use of potent distilled liquor that assisted them in gaining access to goods or land from inexperienced Indians. Consequently, tribes had little time to develop moral and social guidelines for alcohol misuse or deal with the associated negative consequences that result from excessive drinking. 
In a study conducted by Frank, Moore, \& Ames (2000) it was theorized that problematic drinking among AI was a learned behavior that occurred over three distinct phases. Phase 1 was called "Precontact culture: Mind altered states as a social good," substances were used for war-making, enlightenment and used in the context for the 'good of the group'. Phase 2 was referred to as "Initial contact," where little data was available but proposed that, "social harmless drinking may have occurred with no associated violent behavior." Phase 3 was called the "Development of new native drinking cultures" which was plagued by the progressive effects of "high dose binge drinking and strongly associated with physical violence." Historically, phase 3 was representative of a period where alcohol use was marked by lawlessness.

In 1832, tribal leaders requested that the U.S. Congress pass legislation banning the sale of alcohol to Indian people. The prohibition of alcohol was repealed in 1953, thus allowing tribes to sell alcohol on reservations. Today, many tribal reservations have chosen to remain "dry" though the research indicates few differences in reported statistics on "wet" reservations with regard to alcohol morbidity and mortality (Beauvais, 1998). Current reports of alcohol misuse have been cited as one of the largest preventative causes of death, and a major public heath problem among the AV/AN population (CDC, 2008a).

\section{Alcohol Misuse and Alcohol Use Disorders}

A comprehensive literature review indicated that $\mathrm{AU} / \mathrm{ANs}$ have higher prevalence rates of past year alcohol use disorders as compared to other ethnic groups (10.7 vs. 7.6\%). Typically, AI/AN youth become involved with alcohol at an earlier age, misuse it more frequently, and use alcohol in greater amounts as compared to other youth (May \& 
Moran, 1995). Alcohol use among some tribes is more common than among others (Beauvais, 1992). While the 'stereotypic drunken Indian' has left an indelible mark in the public's perception that the vast number of Indians with AUDs are somehow different from other people, thereby further marginalizing the AI/AN race. Findings from a metaanalysis hypothesized that ethnicity increases the risk of alcohol dependence and is increased by Taq1A polymorphism, thus researchers found a small effect when previous studies were compared, thereby doubting the single gene effect or the Mendelian laws of inheritance (Smith, Watson, Gates, Ball, \& Foxcroft, 2007).

In the research of Ehlers (2007) variations in two alcohol metabolizing enzymes called alcohol dehydrogenase (ADH) were examined. ADH converts alcohol to the toxic metabolite called acetaldehyde then reduces it to aldehyde dehydrogenase (ALDH) that is less toxic as acetate, which has been the focus of many genetic studies. ALDH is further divided in the literature as ALDH1, a precursor to vitamin A and ALDH2 that is mostly responsible for the breakdown of acetaldehyde, with both having a 'protective effect' in the body. Many Asians have greater amounts of ALDH2 that is thought to be a protective agent that causes facial flushing when drinking alcohol, thus it has been theorized that AI/ANs originated from the continent of Asia and may carry these different genes but this has not been supported in any study findings (Mulligan, Hunley, Cole, \& Long, 2004). Additionally, no North American Indian tribe or Mission Indians were found to have greater amounts of ALDH2 and only a few Mission Indians were found to have different amounts of ALDH1 and ADH enzymes (Ehlers, 2007).

In another study by Mulligan and colleagues (2004), the issue of genetic predisposition has been addressed by citing the difficulty to genetically identify 
alcoholism, since multiple genes and the environment leads to the trait or expression of a supposed alcohol gene. Other published studies indicate that genes have no role in AUD and that the metabolism of alcohol among all ethnic groups is related to other factors such as a drinking history, family history of alcohol use, social status, social roles, gender differences, and body weight (Collins \& McNair, 2002; SAMHSA, 2002). Prevalence rates for AUD were higher in AI/AN men than women (Beals et al., 2003). Typically, women have about $50 \%$ less of the gastric metabolizing enzyme found in the stomach called $\mathrm{ADH}$ and can reach similar levels of intoxication by drinking about $40 \%$ less than men (York \& Welte, 1994). Historically, women were socialized to either drink less or completely abstain from alcohol use (Fillmore, 1984; Fillmore et al., 1998).

The Behavioral Risk Factor Surveillance System (BRFSS) was a telephone-based survey conducted by IHS and completed between the years of 1997-2000. Survey data focused on health risk behaviors and reported health status indicators that address health disparities that impact AI/ANs (Denny et al., 2003). BRFSS health status indicators are questions about fair to poor health, body mass index (BMI), and awareness of the diagnoses of diabetes. Collected data indicated that AI/ANs more often view their health status as fair to poor, reported higher rates of obesity, and increased awareness of being told of a diabetes diagnosis as compared to other ethnic groups. Findings from the health risk behavior questions on the BRFSS reported that AI/ANs smoke more than other ethnic groups, engage less frequently in exercise, binge drink more often, have fewer pap smears, report medium to high risk for becoming infected with Human Immunodeficiency Virus and Acquired Immune Deficiency Syndrome (HIV/AIDS), and test less frequently for HIV/AIDS when compared with other races (Denny et al., 2003). 
Other studies have reported on the association between substance abuse and less condom use in $\mathrm{AI}$ adults, with higher numbers of females becoming infected with HIV/AIDS (Marsiglia et al., 2006). In 2005, CDC data indicated that AI/ANs rank third among all ethnic minorities for reported cases of HIV/AIDS. Approximately, $29 \%$ of all reported cases are among Al/AN women (CDC, 2008b). BRFSS reported data on binge drinking that was defined as, $\geq 5$ alcoholic beverages on $\geq 1$ occasion during the previous month. Binge drinking among men from Alaska was (30.7\% versus $22.0 \%$ ) while AI men in the Southwest ranked second (30.5\% verses $22.0 \%$ ) for highest binge drinking percentages across the U.S. Overall, binge drinking among AI/ANs was slightly higher as compared to other ethnic groups (23.9\% verses $22.0 \%$ ). Reported drinking and driving are more common among AI/ANs and are reflected in the average annual age adjusted alcoholattributable deaths (AAD) rates that are twice as high compared to the general population (CDC, 2008a). Limitations in the data obtained from BRFSS may include the underreporting of specific health risk behaviors due to racial misclassification of AV/AN ethnicity on state death certificates or language barriers since interviews are not conducted in the AI language (Denny et al. 2003; Epstein, Moreno\& Bacchetti, 1997; Stehr-Green et al., 2002).

Currently, one of the largest annual surveys on alcohol and drug use among AI/ANs is the National Survey on Drug Use and Health (NSDUH) report, which examined substance use and substance use disorders among AV/AN aged 12 and older (USDHHS, 2007c). Findings from this survey indicated that in this population, they were less likely to have past year alcohol use $(60.8 \%$ verses $65.8 \%)$ but were more likely to have a past year alcohol use disorder (AUD) of (10.7\% verses $7.6 \%)$. Male and female 
$\mathrm{AI} / \mathrm{ANs}$ reported higher rates of past year illicit drug use (18.4\% verses $14.6 \%)$ and a past year illicit drug use disorders $(5.0 \%$ versus $2.9 \%)$. Marijuana is the most commonly misused substance when compared to other races (13.5\% versus $10.6 \%$ ), followed by cocaine $(3.5 \%$ versus $2.4 \%)$.

Many of these studies have been useful in providing data on alcohol and other drug use disorders among AV/ANs. Review of the literature indicates that there is limited data on the status of urban AI/ANs with regard to mental health and substance use disorders.

\section{Co-occurring Disorders among the United States Population and AI/ANs}

In response to the growing unmet need to address serious mental health disorders three large epidemiological surveys were carried out in the U.S. the during 1980-1990's. The first large scale survey was the 1980-1985 ECA and comprised five independent study sites sponsored by National Institute of Mental Health (NIMH) in a cooperative agreement among five universities leading to 20,861 participant interviews. Findings provided data on prevalence and incidence rates for specific mental disorders among the general population using the NIMH Diagnostic Interview Schedule (DIS), Version 3, and the DSM-III for psychiatric diagnoses (Bourdon, Rae, Locke, Narrow, \& Reiger, 1992).

Survey estimates identified that one out of five people in the U.S. aged 18 or older has a mental health disorder in any six-month period and that lifetime prevalence rates for all mental illness in the general population were $33 \%$; indicating that one out of three people suffers from mental disorders. The estimates for lifetime prevalence of a substance use disorder were $17 \%$ and alcohol the most commonly abused substance at 14\% (Bourdon et al., 1992). In the general population, lifetime prevalence rates for 
anxiety disorders were $15 \%$, phobia $13 \%$, depression $8 \%$, and antisocial personality disorder reported as $3 \%$. There were no specific statistics on the incidence or prevalence of mental or co-occurring disorders for AI/ANs.

The second large epidemiologic study was the National Comorbidity Survey (NCS) which was an in-person survey of 8,098 respondents carried out between 1990 and 1992 to assess the prevalence and correlates of DSM-III-R disorders in persons ages 15 to 54 (Kessler, Berglund et al., 2002). The NCS was completed in two distinct parts. In Part I, NCS respondents were given the WHO CIDI that was administered by trained laypersons in obtaining diagnoses in accordance with the DSM-III-R and the International statistical classification of diseases and related problems, 10th revision (WHO [ICD-10], 2007. In Part II of the NCS, 5,877 study responders were screened positive for any disorder in Part I, then identified for reporting on correlates of mental disorders in determining role impairments that defined the term serious mental illness (SMI). Study participants were asked to report their prior 12-month functional impairments, defined as; vocational incapacity, 'difficulty holding a job down' because of mental health problems, serious interpersonal difficulties 'isolation from others,' suicidal ideation, or those meeting the criteria for SMI as defined by the National Advisory Mental Health Council of the National Institute of Mental Health (NIMH).

The NCS included screening for severe forms of bipolar disorders, major depression, panic disorders, and non-affective psychoses as these problems likely worsen if left untreated (Kessler, Berglund et al., 2002). The NCS provided no specific data on the incidence and prevalence of SMI in the AI/AN population but was helpful in establishing estimates in the United States population. 
The third largest study in the United States was the National Epidemiologic Survey on Alcohol and Related Conditions (NESARC) that collected data from 43,093 participants in two distinct waves. The first wave collected data from 2001-2002, and the second wave collected data from 2004-2005. This survey examined 12-month comorbidity of DSM-IV drug and AUD in the general population and $9.4 \%$ or 19.4 million will meet established criteria (Grant et al., 2004). The overall purpose of this survey was to examine rates of prevalence, rates for co-occurring disorders specifically alcohol, other drug use disorders by exploring correlates of psychopathology, demographic variables, and treatment seeking behaviors (Stinson, Ruan, Pickering, \& Grant, 2006). Results from this study indicate that AUD and 25 drug use disorders were positive and significant. This survey was less concerned with associated factors that may influence alcohol and drug use disorders and implications for treatment.

The ECA and NCS studies were also useful in defining an operational definition of SMI, which led to the passage of Public Law (P.L.) 102-321 by Congress in 1992. Through this reorganization act, the Alcohol, Drug Abuse, and Mental Health Administration (ADAMHA) was dissolved and a new Federal program called Substance Abuse and Mental Health Services Administration (SAMHSA) came under the umbrella of the U.S. Department of Health and Human Services (USDHHS; Kessler, Berglund et al., 2002). SAMHSA then further defined SMI to include "serious impairment" which is a Global Assessment of Functioning (GAF) score below 60 (Endicott, Spitzer, Fleiss, Cohen, 1976; SAMHSA, 1993). SAMSHA became instrumental in establishing surveys such as the National Household Survey on Drug Abuse (NHSDA) and the National Health Interview Survey (NHIS) that included smaller estimates of SMI for county and 
state block grants (Kessler et al., 2001). Results of the SAMHSA task force identified that SMI was less prevalent in men, more common in a single or divorced person, and not related to variables of education or income (Kessler et al., 1999)

Later, the measures such as the CIDI were then modified to three shorter tools to assess for SMI in 1,000 participants. These three scales included the Structured Clinical Interview for DSM-IV (SCID) that screens for Axis 1 psychiatric diagnoses, K10/K6 scales that measures psychological distress in participants, and the WHO Disability Assessment Schedule (WHO-DAS) that measures the severity of illness related impairments over a 30-day recall. GAF ratings were applied during the SCID to determine SMI while the interviewer remained blind to participant responses obtained from the screening scales (First, Gibbon, Spitzer, Williams, \& Benjamin 1997; Kessler, Andrews et al., 2002; Kessler, Berglund et al., 2002). By adding the K6 scales, the sensitivity was 0.36 , and the specificity was 0.96 (Kessler et al., 2003).

More recently, the National Comorbidity Survey Replication (NCS-R) was administered to 9,282 participants from 2001-2003, and was improved by assessing for a wider range of disorders than the NCS or ECA. The purpose of the NCS-R was to collect data on the lifetime prevalence, age of onset of $D S M-I V$ disorders. Findings concluded that $57 \%$ of Americans would meet the lifetime diagnostic criteria for a DSM-IV psychiatric disorder that typically presents in youth (Kessler et al., 2005).

American Indian Services Utilization and Psychiatric Epidemiology Risk and Protective Factors Project (AI-SUPERPFP) has provided one of the largest estimates on the current prevalence of $D S M-I I I-R$ disorders and help-seeking among 3,084 AI/ANs living on two reservations in the Northern Plains and Southwest. Published results from 
this study identified 12-month higher rates of PTSD and alcohol dependence but lower rates of risk for MDE as compared to the NCS data (Beals et al., 2005). Limitations from the study include that comparison data from National estimates was older, the validity of some tools have not been evaluated, and AI-SUPERPFP used retrospective data collected by laypersons in the context of strict protocols.

Malcolm, Hesselbrock, and Segal (2006) conducted a study to examine the prevalence of co-occurring multiple substance use disorders and course of use among 582 adult AN. This sample did not include AI but identified that the majority of subjects had an AUD and were also dependent on another substances. Dependence on marijuana was most common followed by cocaine and opiates. The combination of alcohol and opiate dependence was more troublesome as their first drink was generally much earlier. Issues of intoxication and withdrawal were more significant when combined with medical and psychological problems. Findings from this study emphasize the need for early intervention aimed towards preventing comorbid drug disorders.

Other studies have supported findings that AI/ANs typically engage in health compromising behaviors at an earlier age when compared to other adolescents (Malcolm et al., 2006; Neumark-Sztainer et al., 1996; USDHHS, 2007). Manifested social, physical, and mental health problems adults experience are often an extension of adolescent learned risk taking behaviors (Kaminer, 2004). Studies of AI adolescents have shown the strong association between substance abuse, delinquency, and risk of suicide (Neumark-Sztainer et al.). The attitudes and influence of peers is a major risk factor for alcohol misuse (Beauvais, 1992). Among all teens, ATOD is related to the 
three most common causes of mortality, which are unintentional injury, homicide, and suicide (CDC, 2008a; Smyth \& Maclachlan, 2004).

In terms of comorbidity, a large study on the prevalence of mental disorders among 736 AI children found that nearly one-fourth of the sample met the 12-month diagnostic criteria for at least one mental disorder and $9 \%$ met the criteria for two or more disorders. Within the same study, AI female caretakers reported prevalence of four psychiatric diagnoses for generalized anxiety disorder, alcohol abuse, MDD, and drug abuse. The most frequently reported diagnoses among these caretakers were alcohol abuse $(63.6 \%)$, drug abuse (20\%), MDD (19.2\%), and generalized anxiety disorders $(5.5 \%)$. Reported limitations of this study were the lack of measures to assess cultural differences (Whitbeck, Hoyt, Johnson, \& Xiaojin, 2006).

A large number of published studies reported high prevalence rates for alcohol abuse among AI; however, little data were available on the types or numbers of substance abuse treatment admissions. The Treatment Episode Data Set (TEDS) provides national information on the number of admissions for substance abuse treatment in a calendar year. In 2009, the most current admission data available was for the year of 2006 . There were a total of $1,917,974 \mathrm{SA}$ admissions and only $2.4 \%$ of those admissions were AI/ANs for the entire year. At the time of admission, AI/ANs reported inhalants as the most commonly abused substance, followed only by alcohol, and then amphetamines (SAMHSA, 2006). Asian, Native Hawaiian or other Pacific Islanders, and AVANs had the fewest reported admissions among all ethnic groups. Conversely, the USDHHS (2007) reported that the three most common illicit drugs used among AI/ANs were marijuana, pain relievers, and cocaine with inhalant use reported less frequently. 


\section{Historical Trauma and Possible Causes for Substance Abuse Disorders}

Historically, numerous governmental acts of genocide on AI have led to the loss of Native culture and practice of rituals through forced socialization of values held among mainstream European Americans (Szlemko, Wood \& Thurman, 2006). Some of the greatest documented traumas in the literature were the first use of biological weapons during the French and Indian wars (1754-1767) where British soldiers knowingly provided blankets to Indians that were infected with the smallpox virus that had previously been quarantined. Once infected with the smallpox virus, Indians suffered a 30\% fatality rate (Henderson et al., 1999). Other traumatic incidences include the 1838 Trail of Tears where Cherokee Indians were forced to leave their home land of North Carolina to Oklahoma where 8,000 out of 17,000 Indians were either starved, exposed to disease, suffered, and died from the lack of medical care (Churchill, 1997).

In the next wave of trauma, the U.S. government established Indian boarding schools from the 1800s until the middle 1900s where AI children were removed from their families, punished for practicing Native rituals and culture, and brainwashed with European Christian values (Churchill, 1997). As a result, AI children were deprived of opportunities that facilitated the transmission of Native cultural values and knowledge, which led to the inability to traditionally parent their own children. Thus, the foundation was laid for the perpetual cycle of intergenerational problems such as alcoholism that was never part of traditional AI communities (Duran, Duran, \& Yellow Horse Brave Heart, 1998; Vernon \& Jumper-Thurman, 2005).

In 1887, other efforts to force assimilation occurred when Congress passed the Dawes or Allotment Act abolishing tribal governments that allowed for the breakup of AI 
reservations containing prosperous farms through the redistribution of land holdings to others. AI were then mandated to enroll in the Bureau of Indian Affairs, accept American citizenship, and in exchange were given a small piece of land often unsuitable to produce food (Kelly, 1990). Again, the U.S. government passed the Termination and Relocation Act of 1954 that removed the Federal rights of 61 tribes, thereby creating groups of 'landless' Indians. This subsidized act caused the immigration of reservation dwelling people into "urban" areas with an agreement that they would not return to rural reservations.

Today, the urbanization of AIs has contributed to the health disparities reported in the current literature. Compared to the general population, urban AI/ANs tended to be poorer, have $38 \%$ higher rates of accidental death, $126 \%$ higher rates of liver disease, $54 \%$ higher rates of diabetes, higher rates of child abuse and neglect cases, and higher numbers of alcohol related deaths (Earle, 2000; Earle \& Cross, 2001; UIHC, 2007; U.S. Census Bureau, 2006). According to Yellow Horse Brave Heart (1999) historical trauma is an accumulation of responsive characteristics that are accumulated over time leading to intergenerational trauma which share similar commonalities among survivors of the Jewish Holocaust and their children. The symptoms from historical trauma are manifested in psychological, social, economic, intellectual, political, physical, and spiritual realms of AI people (Struthers \& Lowe, 2003).

The trauma response features closely mirrors documented AVAN health disparities which demonstrate higher rates of prevalence for morbidity, mortality, cardiovascular disease, hypertension, alcohol abuse, depression, and suicidal behaviors (Yellow Horse Brave Heart \& Debruyn, 1998). Researchers hypothesize that 
disenfranchised grief occurs in persons when a loss cannot be publicly mourned. Many AIs have been characterized as being stoic with less overt or expressed feelings (Doka, 1987, 1989; Yellow Horse Brave Heart \& Debruyn).

As AI children were removed from their homes, restricted, or punished for practicing their ritual and cultural beliefs, there were generations left with traumatic soul wounds. AIs were unable to publicly grieve their losses or perform spiritual ceremonies related to the death of their loved ones. The inability to engage in cultural practices and rituals surrounding bereavement prohibits successful grief resolution and in their absence, maladaptive coping mechanisms many occur if grief is not legitimized (Lenhardt, 1997; Romanoff \& Terenzio, 1998).

AIs frequently report that they experience trauma to loved ones, witness traumatic events, and are victimized more frequently as compared to the overall U.S. population (Manson et al., 2005). Repeated traumatic losses of family through unintentional injuries, alcohol related homicide, and suicide perpetuates feelings of numbness. Layers of current loss trigger past emotions leading to numbing of the psyche and destructive coping mechanisms are manifested as a result of disenfranchised grief and historical trauma (Yellow Horse Brave Heart \& Debruyn, 1998).

There are other loses unrelated to death such as separation from loved ones or loss of a job that often go unrecognized but elicit feelings of grief (Lenhardt, 1997). Other research regarding intergenerational trauma by Koren-Karie, Sagi-Schwartz, \& Joel, (2003) identified that a significant number of children whose parents were Holocaust victims were unable to provide a secure loving infrastructure for the second generation in regard to attachment with significant others. This research further supports that in the 
absence of attachment, parental deficits were likely to be transmitted to the second generation. According to Bar-On and colleagues (1998) insecure attachment may be a risk factor in the development of psychopathology, but under some circumstances is the best coping mechanism in suboptimal child-rearing environments.

The research of Szlemko et al. (2006) reported that many AI youth maintain close relations with extended family who often become part of their peer group as compared to other youth from different ethnicities. Review of the literature supports the notion that peers are influential in development of negative and positive attitudes towards substance abuse, and may serve as an important protective or risk factor. Therefore, the lack of parental monitoring is viewed as either, a protective, or a risk factor. Lack of monitoring, increases the risk for substance abuse and thus, consistent parental monitoring acts as a protective factor in decreasing the risk for delinquency and substance use.

In a study of AI family structure and substance abuse, Lonczak, Fernandez, Austin, Marlatt, and Donovan (2007) found that youth raised in single parent homes were more likely to use alcohol, marijuana, and tobacco. Another factor in the consideration of youth delinquency and substance abuse is the adolescent's perceived perception that there is parental monitoring on a consistent basis and that they can come to their parent with their problems (Mmari, Blum, \& Teufel-Shone, 2010).

\section{Theoretical Framework}

The philosophical underpinnings of the AI/AN worldview are relational by nature with an emphasis on connectedness to the environment and other people (Hill, 2006). Traditionally, there is an emphasis on family and kinship ties, which are of great importance. Native cultures generally share a collectivist orientation; needing to connect 
with others and the environment, which are essential elements in the maintenance of health, and the well being of the group is before the individual (Dodgson \& Struthers, 2005; Lowe, 2002). Balance can only exist when mind, body, and spirit are working in harmonious state. If the body's emotional or spiritual elements are hurting, it will be manifested in physical forms.

According to Dapice (2006) the medicine wheel is a graphic representation of symbolic wholeness (see Figure 1). The components include four directions which are spiritual, emotional, physical and mental are used interchangeable in the representation of the four directions (i.e., north, south, east, west), four seasons of the year (i.e., spring, summer, winter, fall) and the four life givers (i.e., water, air, food, sun). These components are representative of the life cycle and pertain to universal connectedness (Poonwassie \& Charter, 2001).

Social connectedness is part of the self that is subjectively aware of being close to the social world (Lee \& Robbins, 1995; 1998). The lack of social connectedness can influence cognition, perceptions and emotions thereby affecting outward behaviors (Lee \& Robbins, 1998). A recent study by Stewart and colleagues (2009) reported that lower income persons experience a greater sense of isolation and a lower sense of belonging as compared to persons with more income. Loneliness, social support, and social connectedness are predictors of adjustment difficulties (Duru, 2008). 
From a broad American Indian perspective,

the persons being consists of:

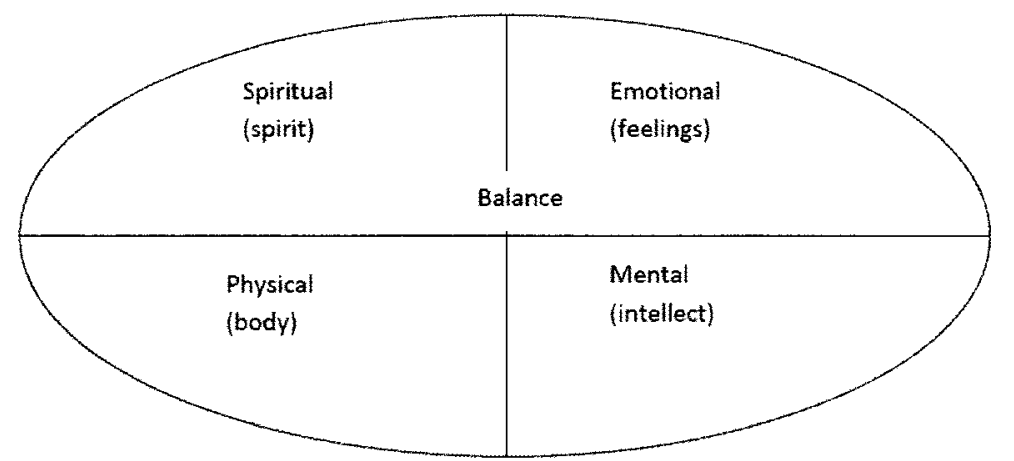

To possess health and wellbeing, these components exist in balance and harmony.

Figure 1. Broad American Indian Worldview Model from Struthers, R., Kaas, M., Hill, D., Hodge, F., DeCora, L., \& Geishirt-Cantrell, B. (2003). Providing culturally appropriate education on Type 2 diabetes to rural American Indians: Emotions and racial consciousness. (Reprinted with permission)

The poverty rates for AIANs are $20.3 \%$ compared to $12.7 \%$ in the general population (U.S. Census, 2006). Metropolitan locations offer more opportunities for employment and education for AI/AN women but due to location, working AI/AN women tend to be isolated and separated from their social supports (Napholtz, 2000). By having a central focus on the family, many employed urban Indian women are more likely to experience bicultural stress from the loss of feeling connected and belonging to a supportive group.

Biculturalism in AI studies is conceptually synonymous with "walking in two worlds" (LaFromboise, Coleman, \& Gerton., 1999; Naphotz, 2000). In a qualitative Native study by Dodgson and Struthers (2005), participants verbalized ways of dealing 
with perceived cultural duality as they tried to compartmentalize by wearing different hats at various times, while trying to "walk in both worlds." Western thinking is linear and Native thought is generally spatial, so the act of trying to mentally compartmentalize is challenging from a Native worldview perspective (Dodgson \& Struthers; Duran \& Duran, 1995). 


\section{Chapter 3}

\section{Methodology}

The purpose of this descriptive study was to gain an understanding of the prevalence of mental health disorders, ATOD, and CODs, among a sample of urban dwelling adult AI/ANs that were seeking behavioral health care at an urban AV/AN community clinic in Southwestern United States. This chapter includes a description of the research aims, design, sample and sampling, data collection procedures, and analytic procedures. The protection of human subjects is also presented.

\section{Specific Aims}

\section{Aim 1}

To characterize a population of $\mathrm{AI} / \mathrm{ANs}$ who are seeking mental health services at an urban Indian community clinic.

\section{$\operatorname{Aim} 2$}

To examine the prevalence of mental health disorders, ATOD, and COD among AI/ANs who are seeking mental health services at an urban Indian community clinic. 
$\operatorname{Aim} 3$

To identify factors that contribute to mental health disorders, ATOD, and COD among AV/ANs who are seeking mental health services at an urban Indian community clinic.

$\operatorname{Aim} 4$

To identify factors that increase the risk of substance use among AI/ANs who are seeking mental health services at an urban Indian community clinic.

\section{Research Design}

A descriptive design using precollected retrospective data was used for this quantitative study. Non-experimental descriptive research allows researchers to observe, document, and describe phenomena (Polit \& Beck, 2008). In general, there are few descriptive or comparative studies addressing the prevalence of mental health disorders, ATOD, and COD among urban AI/ANs.

\section{Sample and Setting}

The sample was extracted from a database of records of patients seen at an urban AI/AN community based healthcare clinic located in Southwestern United States, from January 1, 2008 through February 28, 2010. Retrospective data was collected from individuals $(N=123)$ who had received outpatient behavioral health treatment for a diagnosed mental health, SUD, or a COD. Participant inclusion criteria: verified as tribally enrolled, male or female between the ages of 18 and 65, patients screened with the PHQ-9, and the ASI-NAV. Participant exclusion for this study included the inability to verify tribal enrollment. 


\section{Sample Size}

Cohen (1988) indicates the power of a statistical test relies on the degree that the phenomenon exists, the effect size, the reliability of data, and the significance criterion. The number of subjects needed to reject the null hypothesis refers to the power of the test. If the power is low, failing to reject the null hypotheses may be ambiguous. While notable differences may exist, inadequate sampling can prevent calculated values large enough to exceed the test's critical value. A Type 1 error occurs when the null hypotheses is rejected when in fact no differences exist in the population. A Type 2 error occurs when the null hypothesis should have been rejected, meaning that the treatment or intervention was effective, but the outcome statistic indicated a failure to reject the null hypothesis.

\section{Power, Effect, and Sample Size}

There is no consensus on the approach to compute power and sample size with logistic regression; although as pointed out by Katz (1999) 10 outcomes for each independent variable is appropriate. In logistic regression an estimate of the probability of a certain event occurring is made, rather than detecting the difference or relationship that may be present, such as in linear regression. There are no assumptions made about the dependent variable (DV) and independent variable (IV), the relationship is non-linear, and is not normally distributed (Munro, 2005). Some authors apply the likelihood ratio test or use the test of proportions while various approximations are employed to handle the multivariate case. Authors also advocate the use of the Wald test since the Z-score is routinely used for statistical significance testing of regression coefficients (Demidenko, 2006). This descriptive study did not employ hypothesis testing; thus, the final logistic 
regression model includes statistical significance $(p<0.05)$, where $p$ is from the Wald test for confidence interval (CI) and the odds ratio (OR) describes the strength or nonstrength between two binary data values. The overall statistical significance is tested by the likelihood ratio test $(p<0.1)$ used to demonstrate logistic regression model fit.

\section{Data Collection Procedures}

A case/record abstraction tool was developed to guide in the gathering of information from each patient's behavioral health record. The demographic portion of the chart abstraction form was designed to collect information (demographic and personal data) from which a profile of the sample could be developed. Data included age, gender, ethnicity, and diagnoses.

Socioeconomic scales. Health disparities research has identified that morbidity and mortality rates are notably higher in specific ethnic groups. Wilkinson and Pickett (2006) found that industrialized nations demonstrating wider distributions of wealth had greater overall health disparities. As AI/ANs represent only $2 \%$ of the population and have a greater proportion of health disparities, measures of socioeconomic status were collected within this study (SAMHSA, 1999). Urban Indian programs adhere to some established protocols set by IHS, when patients are assessed for behavioral health problems, thus requiring the use of specific charting forms such as the Brief Assessment, PHQ-9, and the ASI during the patient's course of treatment. In this study, independent variables for socioeconomic scales (SES) were categorically defined in levels of education, employment status, and housing characteristics.

Brief Assessment. The Brief Assessment form includes a mental status exam (MSE) used to document the patient's mental state. The MSE begins with general 
observations and then uses quantitative measures to screen for disorders and cognitive impairments in 10 areas. In a meta-analysis by (McDowell, 2006) the MSE has a sensitivity of $100 \%$, specificity of $78 \%$, and validity of $78 \%$. Scores seemed to be slightly lower for persons with less education. Limitations of the MSE exam include the inability to administer to unconscious patients and subjective reporting, since general observations are scored by the provider that had limited usefulness for the purposes of this study. However, a limited amount of data was abstracted from the Brief Assessment to complete missing information on the patient's health history form related to tribal ethnicity, age, the presenting problem, history of suicide, and the patient's drug of choice at the time of assessment.

Addiction Severity Index-Native American Version. The Addiction Severity Index (ASI) is one of the most widely used assessment tools in the field of addiction. It is administered by a semi-structured interview to gather information about psychosocial factors that contribute to the patient's substance use disorder (Carise \& McLellan, 1999; McLellan \& Marlow-Ferguson, R., 2001). The index has seven subscales which are measured and translated into composite scores (CS). These CS are representative of psychosocial functioning in the areas of: (a) Medical, (b) Employment, (c) Drug and Alcohol Use, (d) Legal, (e) Family History, (f) Family and Social Relationships, and (g) Psychological Problems. An eighth scale was added in the ASI-Native American Version (ASI-NAV) to include spiritual and ceremonial practices (Carise \& McLellan). The ASI is useful for patients with co-occurring disorders with a sensitivity of $85 \%$ and specificity of $80 \%$ (Rikoon, Cacciola, Carise, Alterman, \& McLellan, 2006). ASI results 
may vary among the subscales but Cronbach's alpha ranged from .71 to .90 (McDermott et al., 1996).

The ASI interviewer uses a range of scoring from ' 0 to 9' to rate the patient's need for treatment and severity. An ASI-NAV CS from ' 0 to 1' can be interpreted as no need for treatment and not a problem, ' 2 to 3 ' score means a mild problem with treatment most likely not necessary, scores of ' 4 to 5 ' indicate a moderate problem and some treatment is needed, scores of ' 6 to 7 ' indicate a significant problem that warrants treatment, and a score of ' 8 to 9' is interpreted as a severe problem that requires SA treatment. In the second part of the ASI-NAV interview, the patient is asked to rate their need for treatment on a 5-point scale ranging from ' 0 to 4 '. A score of " 0 " indicated no treatment needed while a score of " 4 " indicates severe need for SA treatment.

The advantage of using the ASI-NAV is that it describes the population of participants; quantifies the level of the substance abuse problems, and is useful in determining the treatment needs of each patient. Conversely, the disadvantage or limitations of the ASI-NAV is the 1-hour length of time taken to complete the measure.

Patient Health Questionnaire-9. The Patient Health Questionnaire (PHQ-9) was adapted from the PRIME-MD and is a self-administered test that measures for depression and other common mental disorders (Spitzer, Kroenke, \& Williams, 1999). This measure is similarly scored to the diagnostic criteria of the $D S M-I V$ and is numerically scored " 0 (meaning not at all) to 3 (nearly every day)," to rate the level of depression (Kroenke, Spitzer, \& Williams, 2001). The sensitivity was $88 \%$, specificity was $88 \%$ with good validity. Limitations to the PHQ-9 are that some lower levels of depression may not be diagnosed, but useful in identifying COD in the clinical setting. 


\section{Data Analysis}

The SPSS statistical analysis program was utilized to analyze the data collected in this study. Initial descriptive statistics were computed (mean, median, mode and standard deviations) for numerical variables to characterize the sample. The $t$-test was applied in comparing gender differences and age at first use of the most commonly abused substances. The Mann-Whitney $U$ test was useful in analyzing differences in two or more independent groups: PHQ-9 scores by gender. Calculating the $U$ statistic is considered stronger than the median test (Polit \& Beck, 2008). The Chi-Square test or $\chi^{2}$ was used in this study to determine proportional differences among variables when gender, mental health, ATOD, and COD was analyzed.

Pearson's correlation was computed to measure the association between continuous variables. One-way analysis of variances (ANOVA) was conducted to evaluate significant mean differences of smoking status by age and educational level. Logistic regression was used to examine the factors that increased the risk of substance abuse.

\section{Dependent Variables}

The dependent variable (DV) can be the presence or absence of a predicted outcome within the selected cohort (Polit \& Beck, 2008). In this descriptive study, the DV is having a diagnosed mental health disorder, ATOD, or COD among AI/ANs as documented in the medical charts utilizing ICD-9 or DSM-IV codes.

\section{Independent Variables}

Descriptive statistics were computed to summarize the means and percentages of the independent variables (IV) described in this study. Demographic variables of 
ethnicity, gender, employment, education, housing status, ASI-NAV composite scores and PHQ-9 depression scores were considered IVs in the study.

\section{Limitations}

A limitation of the study is that it relies on pre-collected data. Inconsistent, inaccurate or missing data resulted in a smaller sample size than originally anticipated. Although every attempt was made to find the first documented incidence of mental health, substance abuse, and co-occurring mental health issues, there was no contact with patients or nursing staff, only the documented data in the medical record was available for recording of variables. There may be inconsistencies in coding for psychiatric diagnoses or $I C D-9$ codes.

\section{Human Subjects Protection}

To ensure protection of all subjects' freedom from intrinsic risk or injury, all human subject protection considerations were utilized in this study. This study employed retrospective chart extraction, and all patient identification was removed, thus no informed consent was required. During the process of secondary data collection, all information was stored in a locked cabinet and only accessible to the principle researcher. All patients' identifying data were removed and substituted by codes. Methods of data collection and study plans were reviewed and approved by the governing tribal council for retrospective chart abstraction at the urban Indian clinic. Approval for the study was obtained from the Institutional Review Board (IRB) of the University of San Diego and the National IRB at Indian Health Service Headquarters in Rockville, MD (Appendix A and B). 


\section{Chapter 4}

\section{Study Results}

The purpose of this descriptive study was to gain an understanding of the prevalence of mental health disorders, alcohol, tobacco, and other drug use (ATOD), and co-occurring disorders (COD), among a sample of urban dwelling AI/ANs seeking behavioral health care from an urban AI/AN community clinic in Southwestern United States. In this chapter, results are presented including a profile of the sample followed by each research question and the results of the analysis.

\section{Characteristics of the Sample}

Data were collected through the retrospective audits of 256 patient records for individuals receiving behavioral health care services during 2008 through 2010 at a large urban Indian health program located in the Southwestern United States. Patient history forms, screening interviews, and scores form the ASI-NAV, PHQ-9, DSM-IV diagnoses, and $I C D-9$ codes were used in the collection of data for determining prevalence rates for past year substance use, lifetime substance abuse, and mental health diagnoses. Based upon inclusion criteria, the sample was comprised of 123 individuals between the ages of 18 to 65 verified as $\mathrm{AN}$ or members of a federally recognized AI tribe. There were 43 
ASI-NAV subject interviews scored and translated into CS, thus providing data related to the patient's substance abuse history. PHQ-9 depression scores were obtained for 46 subjects from the larger sample.

Aim 1: To characterize a population of AI/ANs who are seeking mental health services at an urban Indian community clinic. Descriptive characteristics are presented in Table 1. Descriptive Characteristics

As shown in Table 1, the sample was approximately evenly distributed based on gender (64 males, 59 females), with a mean age of $38.94(S D=11.01)$ years, range 1865. There was no statistical difference in age by gender $\left(M_{\text {male }}=38.84\right.$ years; $M_{\text {female }}=$ 39.05 years). The majority subjects were American Indian (95.9\%) and had completed at the minimum a high school education $(81.9 \%)$. 
Table 1

Descriptive Characteristics $(N=123)$

\begin{tabular}{|c|c|c|}
\hline & $n$ & Percent \\
\hline \multicolumn{3}{|c|}{ Gender } \\
\hline Male & 64 & 52.0 \\
\hline Female & 59 & 48.0 \\
\hline \multicolumn{3}{|c|}{ Race } \\
\hline American Indian & 118 & 95.9 \\
\hline Alaskan Native & 5 & 4.1 \\
\hline \multicolumn{3}{|c|}{ Level of Education } \\
\hline Grade School & 6 & 5.2 \\
\hline Some High School & 15 & 12.9 \\
\hline High School Grad & 44 & 37.9 \\
\hline Some College & 40 & 34.5 \\
\hline College Grad & 3 & 2.6 \\
\hline Post Grad & 8 & 6.9 \\
\hline \multicolumn{3}{|c|}{ Employment } \\
\hline Unemployed & 70 & 60.3 \\
\hline Part Time & 18 & 15.5 \\
\hline Full Time & 19 & 16.4 \\
\hline Disabled & 7 & 6.0 \\
\hline Student & 2 & 1.7 \\
\hline \multicolumn{3}{|c|}{ Housing } \\
\hline Rent & 36 & 31.3 \\
\hline Own & 3 & 2.6 \\
\hline Homeless & 21 & 18.3 \\
\hline Live with family & 35 & 30.4 \\
\hline Treatment facility & 12 & 10.4 \\
\hline Live with friend(s) & 8 & 7.0 \\
\hline
\end{tabular}


Aim 2: To examine the prevalence of mental health disorders, ATOD, and COD among

AI/ANs who are seeking mental health services at an urban Indian community clinic.

Frequencies for the DSM-IV and ICD-9 codes for 122 out of 123 subjects

(range $=1$ to 8 diagnostic codes). All substance abuse and psychiatric codes were translated and evaluated (see Figure 2).

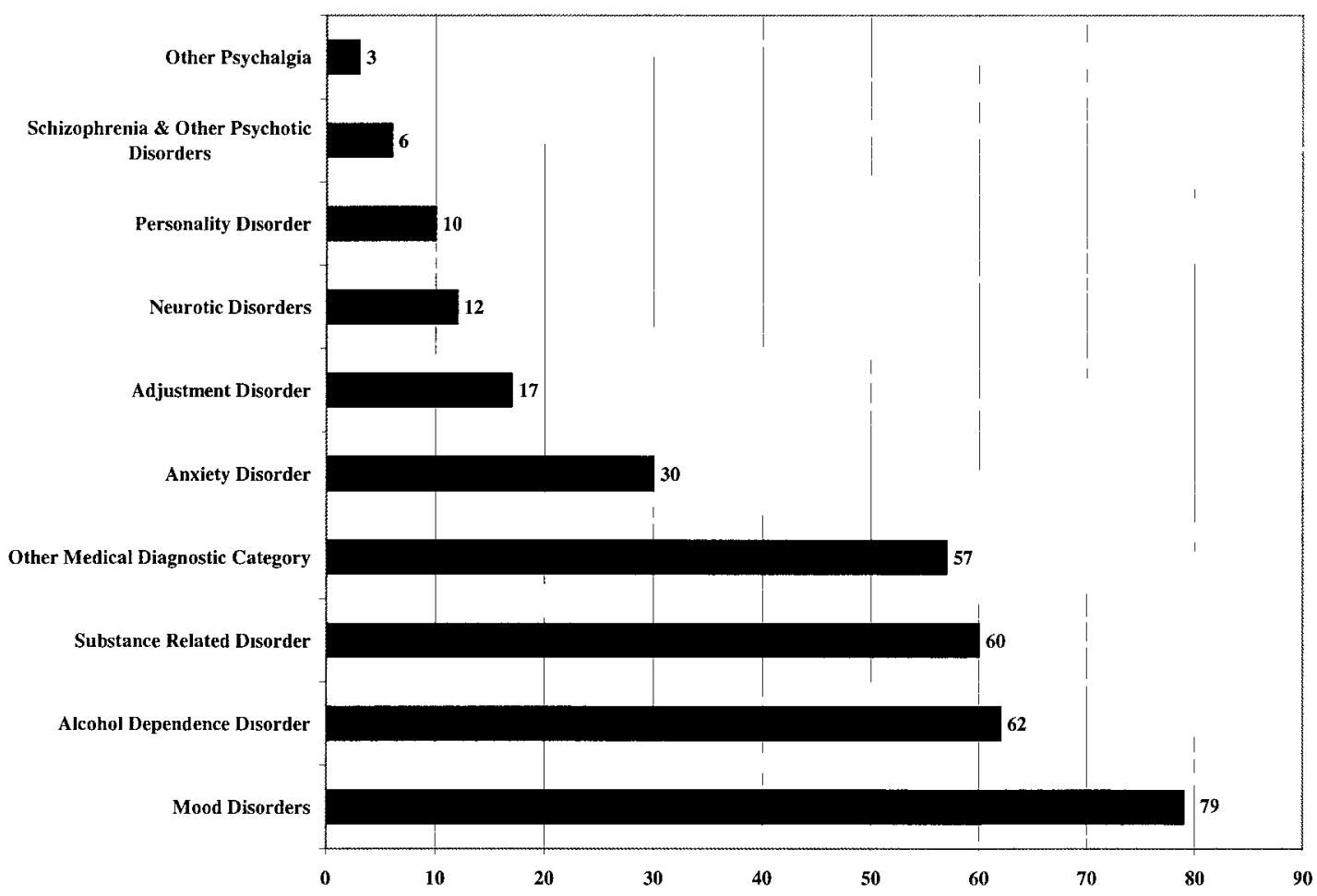

Figure 2. Frequency of DSM-IV Categories $(n=122)$ 
The three most frequently occurring ICD-9 codes are listed in Table 2 below. In addition, the respective prevalence rates are listed. One chart was devoid of $I C D-9$ or DSM-IV classification; thus calculations were based on 122 patients.

Table 2

Highest Frequency of Reported ICD-9 Codes $(n=122)$

ICD-9 Code

Alcohol Dependence

Major Depressive Disorder - Recurrent

Moderate

Methamphetamine
303.9

296.32

304.4 n Prevalence

$61 \quad 50 \%$

$38 \%$

46

23

$19 \%$

To understand the dispersion of $D S M-I V$ psychiatric disorders, individual $I C D-9$ codes were categorized by their characteristic features according to established guidelines (Owens, Myers, Elixhauser, \& Brach, 2007). Multiple alcohol-related disorders exist, alcohol dependence, specifically $I C D-9$ code 303.9 , was the most commonly occurring classification. Grouping codes into overarching DSM-IV categories, multiple depressionrelated $I C D-9$ codes outnumbered alcohol dependence diagnoses, yielding a higher prevalence of psychiatric mood-related disorders.

Due to the limited number of ASI-NAV screenings, any of the $123 \mathrm{AI} / \mathrm{ANs}$ reporting substance use histories were identified and included on this survey. The patient's health history form, Brief Assessment, and ASI-NAV had questions regarding the most frequently abused substances. In describing particular substances among 123 
urban AI/ANs visiting the clinic for behavioral health-related problems, Table 3 lists the results by gender.

Table 3

Sample Characteristics by Reported Incidence of Substance Abuse and Gender $(N=123)$

\begin{tabular}{|c|c|c|c|c|}
\hline Substance & Male & Female & Reported & Percent of \\
\hline & & & Users & Total \\
\hline Alcohol (any use) & 50 & 47 & 97 & $78.9 \%$ \\
\hline Alcohol ( 5 or more drinks/day) & 16 & 16 & 32 & $26.0 \%$ \\
\hline Heroin & 10 & 7 & 17 & $13.8 \%$ \\
\hline Methadone & 4 & 2 & 6 & $4.9 \%$ \\
\hline Other Opiates/Analgesics & 5 & 4 & 9 & $7.3 \%$ \\
\hline Barbiturates & 2 & 0 & 2 & $1.6 \%$ \\
\hline Sedatives/Hypnotics/Tranquilizers & 5 & 4 & 9 & $7.3 \%$ \\
\hline Cocaine & 25 & 19 & 44 & $35.8 \%$ \\
\hline Amphetamines & 32 & 24 & 56 & $45.5 \%$ \\
\hline Cannabis & 38 & 26 & 64 & $52.0 \%$ \\
\hline Hallucinogens & 17 & 7 & 24 & $19.5 \%$ \\
\hline Inhalants & 5 & 1 & 6 & $4.9 \%$ \\
\hline Polysubstance per day & 27 & 16 & 43 & $35.0 \%$ \\
\hline
\end{tabular}

The substance abuse counselor or licensed behavioral health staff identified patients that were screened for depression using the PHQ-9 based on their clinical 
judgment. During 2008-2010, the PHQ-9 was completed in 46 charts to measure the severity level of depression or to make a tentative diagnosis of depression (Table 4).

Table 4

PHQ-9 Results $(n=46)$

\begin{tabular}{|c|c|c|c|c|}
\hline & \multirow[b]{2}{*}{ Not at all } & \multicolumn{3}{|c|}{$\begin{array}{l}\text { More } \\
\text { than }\end{array}$} \\
\hline & & $\begin{array}{c}\text { Several } \\
\text { days }\end{array}$ & $\begin{array}{l}\text { half the } \\
\text { days }\end{array}$ & $\begin{array}{c}\text { Nearly } \\
\text { every day }\end{array}$ \\
\hline Little interest/pleasure in doing things & 10 & 12 & 12 & 12 \\
\hline Feeling down, depressed, or hopeless & 13 & 15 & 4 & 14 \\
\hline Trouble falling or staying asleep or sleep too & 12 & 13 & 6 & 15 \\
\hline \multicolumn{5}{|l|}{ much } \\
\hline Feeling tired/little energy & 11 & 15 & 3 & 17 \\
\hline Poor appetite/overeating & 17 & 11 & 7 & 11 \\
\hline Feel bad about self & 10 & 15 & 7 & 14 \\
\hline Trouble concentrating & 15 & 11 & 10 & 10 \\
\hline Move/speak slow or opposite & 28 & 5 & 8 & 5 \\
\hline \multirow[t]{3}{*}{ Suicidal thoughts/harm to self } & 35 & 3 & 6 & 2 \\
\hline & Not at all & Somewhat & Very & Extremely \\
\hline & difficult & difficult & difficult & difficult \\
\hline How difficult are these problems & 9 & 18 & 14 & 4 \\
\hline
\end{tabular}


The PHQ-9 total score was calculated using the model presented in Kroenke et al (2001). The sum of 10 items was then categorized into levels or severity of depression. For the 46 subjects who had a PHQ-9 in their medical chart, the results from depression categories are displayed on Table 5 .

Table 5

$P H Q-9$ Severity Scores $(n=46)$

\begin{tabular}{lcc}
\hline & Frequency & Percent of Total \\
\hline None & 13 & $28.3 \%$ \\
Mild & 10 & $21.7 \%$ \\
Moderate & 5 & $10.9 \%$ \\
Moderately Severe & 8 & $17.4 \%$ \\
Severe & 10 & $21.7 \%$ \\
\hline
\end{tabular}

The scores obtained from the PHQ-9 were compared with diagnostic ICD-9 codes as noted from review of the clinician's notes located in the patient's chart. The PHQ-9 categories were classified, with 'none' and 'mild' considered as having no to mild depressive symptomatology on screening. The ICD-9 diagnostic codes were scanned for those codes falling in the range of 296.20-296.99 as having a diagnosed depressive disorder. In this study, dysthymia (ICD-9 code 300.40) as a mood disorder was not considered as a depressive disorder. The results are presented in Table 6. 
Table 6

Diagnosis of Depression: PHQ-9 Scores versus Clinician Documentation $(n=46)$

\begin{tabular}{lccc}
\hline & Clinical & No Clinical & \\
& Diagnosis & Diagnosis & Total \\
\hline PHQ-9 Moderate-Severe Depression & 18 & 5 & 23 \\
PHQ-9 No-Mild Depression & 10 & 13 & 23 \\
Total & 28 & 18 & 46 \\
\hline
\end{tabular}

There were more documented $I C D-9$ codes for depression than available depression scores from PHQ-9 screenings. A statistical expression was generated for concordance of depression codes and scores from PHQ-9 to survey whether the PHQ-9 was accurate in diagnosing depression, thus a two-by-two table was constructed to compare positive and negative test results with the diagnostic codes in the chart. Sensitivity measured the agreement between a positive test result (e.g., MDD) and a diagnostic code for depression (sensitivity $=64.29$ ). Specificity measures the ability of the test to yield a negative result when, in fact, no diagnostic code is warranted $($ specificity $=72.22)$.

For those subjects who had a completed PHQ-9 in their chart $(n=46)$, the prevalence rate for depression was $60.87 \%$. Evaluating the 122 subjects whose charts included $I C D-9$ and $D S M-I V$ codes, the prevalence of depression with diagnostic depression codes was $64.75 \%$. Using these data, the positive predictive value was calculated at $78 \%$ and negative predictive value of $57 \%$. 


\section{Gender}

There was no difference between genders and any PHQ-9 questions, except for poor appetite or overeating, where women scored significantly lower (Mann Whitney $U=170, p<.05)$. There was no statistically significant correlation between the PHQ-9 total score and age $(r=.172, p>.05)$

Gender differences and the most frequently abused substances are presented as age at first use by gender $(N=123)$ in Table 7 . Many patients did not return to the clinic for further mental health or substance abuse treatment depending on the individual's circumstances (e.g., attending an outside treatment program, transient population). 
Table 7

Chart Documentation of Age at First Use by Gender $(N=123)$

\begin{tabular}{|c|c|c|}
\hline Substance & $\begin{array}{c}\text { Male } \\
\text { (Range) }\end{array}$ & $\begin{array}{l}\text { Female } \\
\text { (Range) }\end{array}$ \\
\hline *Alcohol (any use) & $13.24(5-22)$ & $15.49(7-35)$ \\
\hline Alcohol ( 5 or more drinks/day) & $13.00(6-18)$ & $17.13(11-35)$ \\
\hline Heroin & $19.80(8-35)$ & $26.43(9-48)$ \\
\hline *Methadone & $21.00(15-35)$ & $53.00(46-60)$ \\
\hline Other Opiates/Analgesics & $27.60(8-44)$ & $36.75(26-52)$ \\
\hline Barbiturates & $16.00(12-20)$ & NA \\
\hline Sedatives/Hypnotics/Tranquilizers & $21.00(12-39)$ & $24.75(14-41)$ \\
\hline Cocaine & $18.16(9-33)$ & $22.26(13-46)$ \\
\hline Amphetamines & $19.22(11-42)$ & $19.88(11-43)$ \\
\hline *Cannabis & $14.37(7-25)$ & $16.81(11-35)$ \\
\hline Hallucinogens & $15.06(8-21)$ & $16.00(13-21)$ \\
\hline Inhalants & $16.60(10-33)$ & $13.00(13)$ \\
\hline Polysubstance per day & $16.81(12-35)$ & $16.81(11-49)$ \\
\hline
\end{tabular}

Results from $t$-tests revealed significant differences between gender and age at first use as follows: alcohol $(t[96]=-2.321, p=.022)$, methadone $(t[5]=-3.842$, $p=.018)$, and cannabis $(t[63]=-2.053, p=.044)$. In all cases with significant differences, males began their substance abuse at an earlier age as compared to women. 
Men reported substance use at an earlier age among all categories, with the exception of inhalants, but only three substances were statistically significant (i.e., alcohol, methadone, cannabis). Six people reported inhalant use. A $t$-test for significance for this category could not be performed as only one woman reported using inhalants.

There was no recent data readily available in the clinic on mental health and substance abuse by gender prior to chart abstraction. Associated factors related to gender in broader $D S M-I V$ categorization of mental health and SUD diagnoses are shown in distribution of disorders seen in the clinic's population (Table 8). This data is useful in terms of planning and implementing an effective behavioral health program to meet the needs of incoming patients.

Table 8

Mental Health Disorders and Substance Abuse by Gender

\begin{tabular}{lcc}
\hline & $\begin{array}{c}\text { Male } \\
(\% \text { of Total })\end{array}$ & $\begin{array}{c}\text { Female } \\
(\% \text { of Total })\end{array}$ \\
\hline Mental Health Disorders & 46 & 51 \\
Substance Abuse Disorders & $(73.0 \%)$ & $(86.4 \%)$ \\
& 52 & 41 \\
Co-occurring Disorders & $(82.5 \%)$ & $(69.5 \%)$ \\
& 35 & 33 \\
Total & $(55.6 \%)$ & $(55.9 \%)$ \\
& 63 & 59 \\
\hline
\end{tabular}


Chi-square indicated no significant difference between genders on mental health disorders $\left(\chi^{2}=1.501, p>.05\right)$, substance use disorders $\left(\chi^{2}=1.501, p>.05\right)$, or cooccurring disorders $\left(\chi^{2}=1.501, p>.05\right)$.

\section{Smoking (Tobacco Use)}

A one-way ANOVA was conducted to evaluate significant mean differences of smoking status (i.e., current smoker, quit smoking, never smoked) by age and educational level $(n=110)$. Patients who had reportedly quit smoking were significantly older than current smokers $\left(M_{\text {Quit }}=43.0\right.$ years vs. $M_{\text {Smoke }}=35.3$ years, $\left.F[2,107]=4.577, p=.012\right)$, but neither group was significantly different from non-smokers ( $M=39.5$ years). Those patients who reportedly quit smoking and those who had never smoked had significantly higher levels of education (median $=$ some college) than those who were active smokers $($ median $=$ high school graduates, $F[2,103]=5.972, p=.004)$.

Findings from chi-square analysis indicated no significant difference between genders on smoking status $\left(\chi^{2}=1.501, p>.05\right)$. Table 9 shows the distribution of genders by smoking status. 
Table 9

Distribution of Gender by Smoking Status

\begin{tabular}{lccc}
\hline & Male & Female & Total \\
\hline Non-Smoker & 20 & 15 & 35 \\
Smoker & 21 & 27 & 48 \\
Quit Smoking & 14 & 13 & 27 \\
Total & 55 & 55 & 110 \\
\hline
\end{tabular}

Thirty Day and Reported Years of Substance Use

In the ASI-NAV interview, subjects were asked to report the number of days of alcohol and drug use for the past 30 days and the number of years of regular use. Two alcohol questions were designed to capture the frequency of any drinking as well as binge drinking. Eleven substances other than alcohol were specifically listed (Table 10). 
Table 10

Substance Abuse as Reported on the ASI-NAV $(n=43)$

\begin{tabular}{|c|c|c|}
\hline Substance & $\begin{array}{c}\text { Reports Use in Past } 30 \\
\text { Days }(n)\end{array}$ & $\begin{array}{l}\text { Reports of } 1 \text { or More } \\
\text { Years Regular Use }(n)\end{array}$ \\
\hline Alcohol (any use) & 29 & 35 \\
\hline Alcohol ( 5 or more drinks/day) & 21 & 26 \\
\hline Heroin & 2 & 6 \\
\hline Methadone & 0 & 1 \\
\hline Other Opiates/Analgesics & 5 & 4 \\
\hline Barbiturates & 0 & 0 \\
\hline Sedatives/Hypnotics/Tranquilizers & 1 & 4 \\
\hline Cocaine & 5 & 13 \\
\hline Amphetamines & 7 & 19 \\
\hline Cannabis & 13 & 25 \\
\hline Hallucinogens & 1 & 6 \\
\hline Inhalants & 0 & 0 \\
\hline Polysubstance per day & 7 & 9 \\
\hline
\end{tabular}

Thirty Day and Lifetime Prevalence of Suicide and Violence by Gender

The ASI-NAV psychiatric domain screened for 30-day and lifetime incidences of suicide ideation, attempts of suicide, and violent behavior. These responses were useful to the interviewer in determining whether recent psychiatric issues were contributory to 
the patient's psychosocial functioning, even if they occurred in the context of alcohol or drug use. During the substance abuse screening interviews, patients reporting suicidal ideation were referred out for urgent psychiatric evaluation.

Forty-three ASI-NAV responders answered questions relative to substance misuse, suicide, and violence. To gain a greater understanding of how suicide and violence impacts urban $\mathrm{AI} / \mathrm{ANs}$, abstracted data from patient health history forms also contained questions pertaining to past substance abuse, suicide, and violence, thus increasing the number from 43 ASI-NAV responders to approximately 84 subjects. Table 11 shows reported incidences of 30-day and lifetime reports of suicide and violence by gender. 
Table 11

Thirty Day and Lifetime Reports of Suicide and Violence

\begin{tabular}{|c|c|c|c|c|}
\hline & $\begin{array}{c}\text { Male } \\
\text { (Prevalence) }\end{array}$ & $\begin{array}{c}\text { Female } \\
\text { (Prevalence) }\end{array}$ & $\begin{array}{l}\text { Reported \# } \\
\text { Incidents }\end{array}$ & $\begin{array}{l}\text { Overall } \\
\text { Prevalence }\end{array}$ \\
\hline $\begin{array}{l}\text { Suicidal Ideation - Last } 30 \\
\text { days }\end{array}$ & $\begin{array}{c}4 * \\
(8.8 \%)\end{array}$ & $\begin{array}{c}11 * \\
(28.2 \%)\end{array}$ & 15 & $17.9 \%$ \\
\hline Suicidal Ideation - Lifetime & $\begin{array}{c}17 \\
(37.0 \%)\end{array}$ & $\begin{array}{c}20 \\
(52.6 \%)\end{array}$ & 37 & $44.0 \%$ \\
\hline $\begin{array}{l}\text { Suicide Attempt - Last } 30 \\
\text { days }\end{array}$ & $\begin{array}{c}0 * \\
(0.0 \%)\end{array}$ & $\begin{array}{c}4^{*} \\
(10.5 \%)\end{array}$ & 4 & $4.8 \%$ \\
\hline Suicide Attempt - Lifetime & $\begin{array}{c}7 * \\
(15.2 \%)\end{array}$ & $\begin{array}{c}13 * \\
(34.2 \%)\end{array}$ & 20 & $23.8 \%$ \\
\hline $\begin{array}{l}\text { Problems controlling violent } \\
\text { behavior - Last } 30 \text { days }\end{array}$ & $\begin{array}{c}8 \\
(17.8 \%)\end{array}$ & $\begin{array}{c}9 \\
(24.3 \%)\end{array}$ & 17 & $20.7 \%$ \\
\hline $\begin{array}{l}\text { Problems controlling violent } \\
\text { behavior - Lifetime }\end{array}$ & $\begin{array}{c}35 \\
(71.4 \%)\end{array}$ & $\begin{array}{c}34 \\
(82.9 \%)\end{array}$ & 69 & $76.7 \%$ \\
\hline
\end{tabular}

Significant differences between genders were seen in the reported incidences of suicidal ideation in the last 30 days (Fisher's Exact; $p=.025$ ), and suicide attempts, both in the last 30 days (Fisher's Exact; $p=.040)$ and lifetime $(\chi 2=4.128, p=.042)$. In all cases of significance, women reported a higher-than-expected number of incidences, and men reported lower-than expected number of incidences. While an appreciable 
difference was seen in the lifetime incidence of controlling violent behavior, no statistical difference could be detected $(\chi 2=1.650, p=.199)$.

Statistical $t$-tests were computed to examine whether there were age differences in suicidal behavior (ideation or attempt) or problems controlling violence (30 days $n=82$; lifetime $n=90$ ). There were no significant differences identified in the data. Difficulty controlling violent behavior was then compared to depression as measured by the PHQ-9 ( $n=46)$ and yielded no significant differences, whether the difficulty to control violence occurred in the last 30 days or at any point in their lifetime. There were no significant differences between gender and violence (30 days $n=82$; lifetime $n=90$ ). Findings from the analysis of data also identified that there was no difference between violence and a clinical diagnosis of depression $(n=122)$.

Aim 3 To identify factors that contribute to mental health disorders, $A T O D$, and COD among AI/ANs who are seeking mental health services at an urban Indian community clinic.

The revised ASI-NAV was administered to only 43 subjects receiving behavioral health care services over the preceding 3-year period (35.0\%). Two scores were collected from revised ASI-NAV: (a) composite scores (CS) and (b) the interviewer's score. McLellan et al. (1985) acknowledged that the number of specific items comprising total ASI CSs might not be equal; however, each of the seven domains were weighted equally to ensure internal validity and consistency with Cronbach's alpha of 0.70 . 
In this study, ASI-NAV CS were obtained for the eight domains representing psychosocial functioning as defined by medical, employment, alcohol use, drug use, legal, family, psychiatry, and spiritual; higher CSs represent more dysfunction in each domain. Subjects coded with a mental health $I C D-9$ code had significantly higher CSs in the psychosocial functioning area of psychiatry ( 0.597 vs. $0.329, t=-2.720, p=.01)$ than those who did not have a mental health $I C D-9$ code.

AI/ANs who were identified as having a co-morbid SUD and a mental health diagnoses by ICD-9 codes also had significantly higher psychiatry CS on the ASI-NAV, than people who did not have co-morbidity ( 0.519 vs. $0.327, t=-2.962, p<.01)$. There were no other significant differences in the remaining six ASI-NAV CS domains with regard to mental health disorders or co-morbid diagnoses.

\section{Interviewer's Rating of Need for Treatment by ASI-NAV CS}

In recognizing cultural and gender differences in chemically dependent patients, the interviewer's perception of the severity of the SUD may differ from the patient. During the substance abuse assessment at the clinic, the interviewer rated the need for counseling in each of the eight ASI-NAV CS categories/domains. Severity profiles for CSs ranged from 0 (no need for counseling) to 9 (most severe need for counseling). A comparison by gender yielded no significant differences ( $t$-test range from -0.396 to $1.156, p>.05)$, therefore, the interviewer rating of severity was not significantly different by gender. The overall results are displayed in Figure 3 below. 


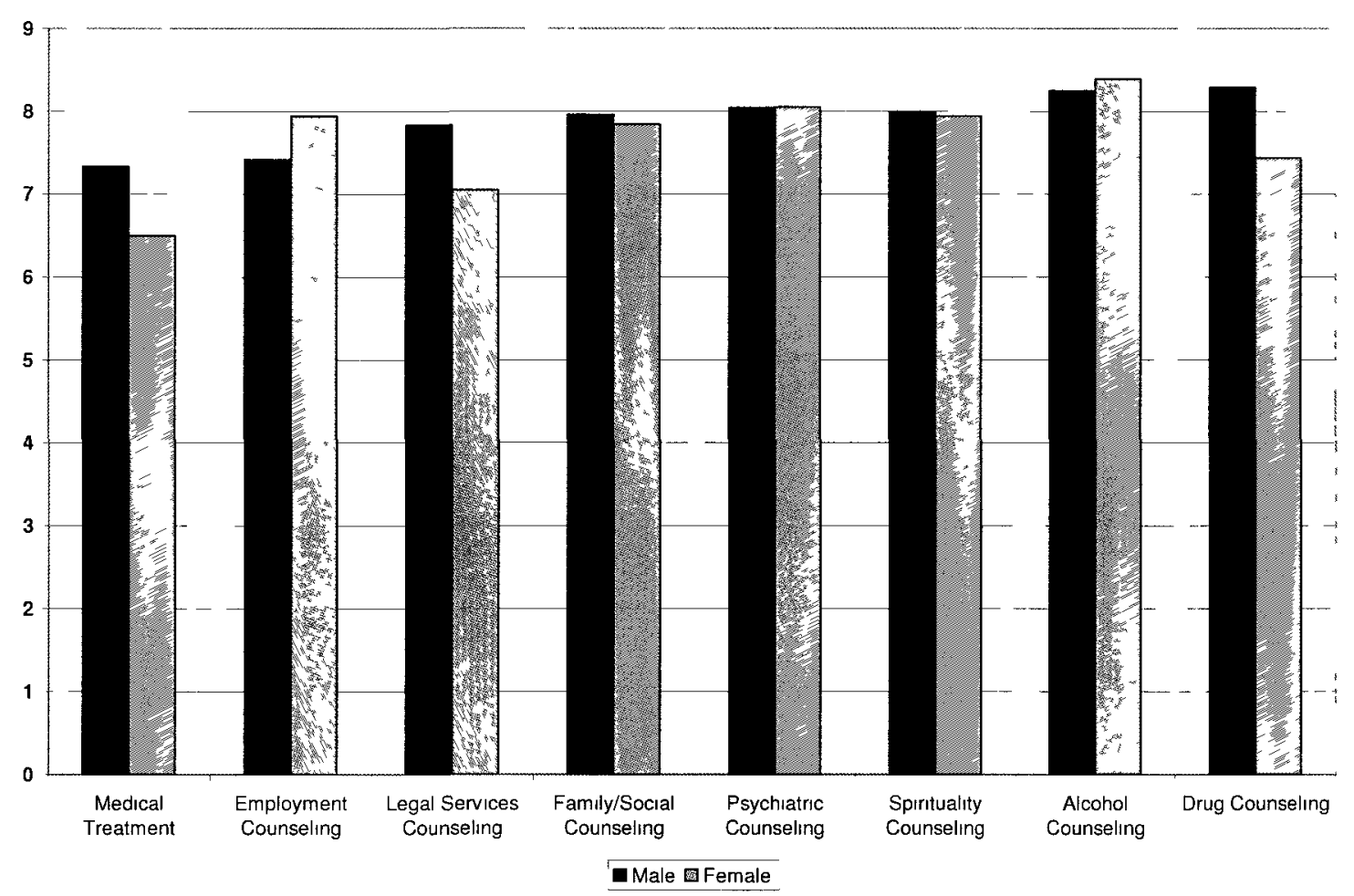

Figure 3. Interviewer Severity Ratings of the Subjects Need for Treatment on ASI-NAV

\section{Patient's Rating of Need for Treatment by ASI-NAV CS}

During the substance abuse assessments, patients were asked to rate their perceived severity of the problem and rate their need for treatment on the ASI-NAV in each of the domains. Unlike the clinician's subjective severity ratings, patients scored their severity from 0 (minimal rating) to 4 (severe rating). Figure 4 presents the results of this analysis. While patients perceived their need for treatment higher than the severity of the domain, with the exception of employment and medical, only the need for alcohol treatment was statistically higher than the severity of the problem (Wilcoxon Signed Rank Test, $z=-2.032, p<.05)$. 


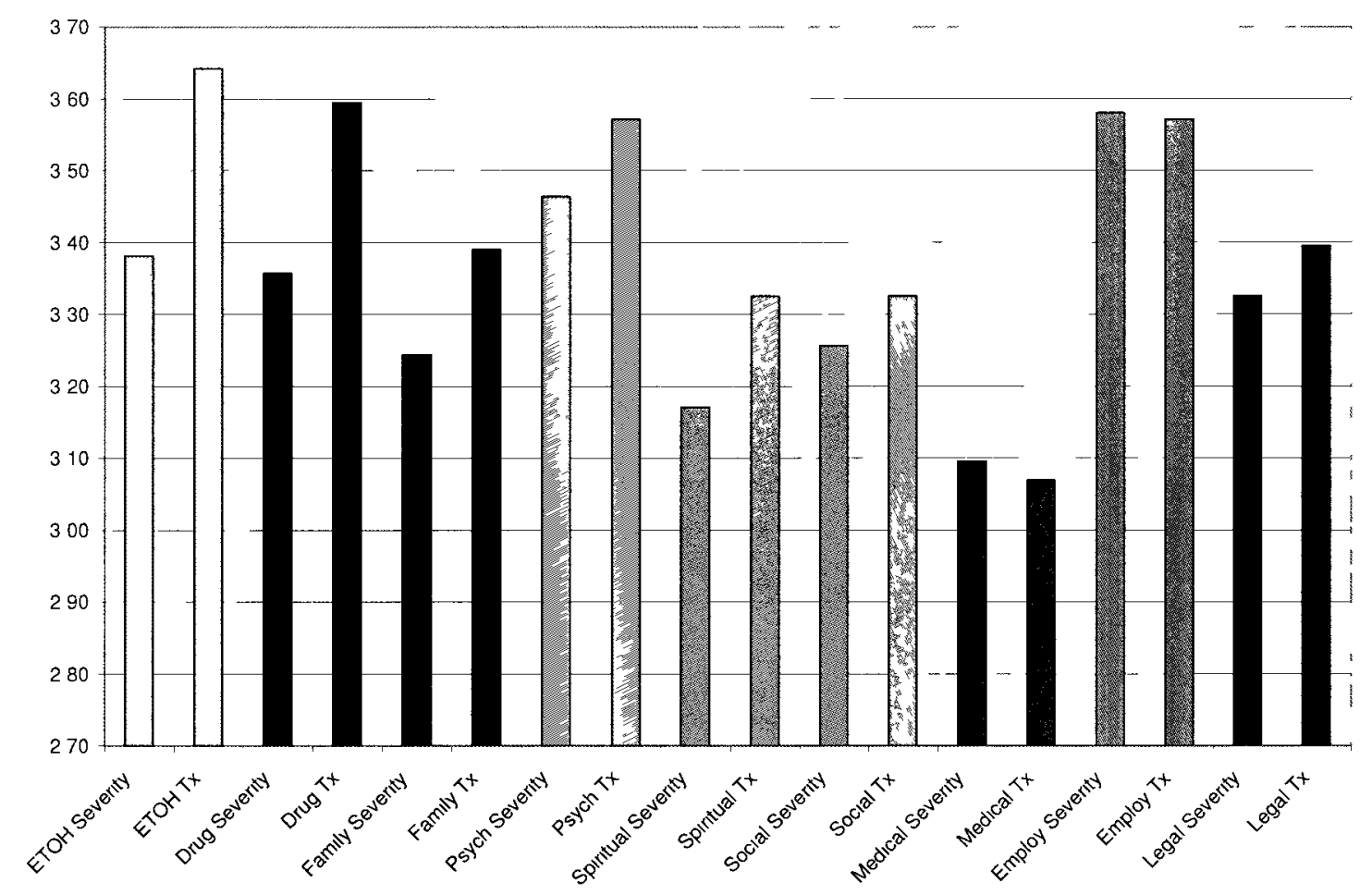

Figure 4. Patient's Rating of Severity and Need for Counseling Services

\section{Correlation among ASI-NAV Composite Scores}

As an individual's misuse of substances progresses, areas of psychosocial functioning become more complex by independent or overlapping problems such as the ability to maintain employment or family relations. Since ASI-NAV CS represents different variables of psychosocial functioning, all 43 ASI-NAV CS were collected and analyzed for any significant correlating factors, thus a correlational matrix was computed (see Table 12). 
Table 12

Pearson r Correlations among the ASI-NAV Composite Scores $(n=43)$

Medical Employment Alcohol Drugs Legal Family Psychiatry

\begin{tabular}{|c|c|c|c|c|c|c|c|}
\hline Medical & 1 & & & & & & \\
\hline Employment & -.143 & 1 & & & & & \\
\hline Alcohol & -.079 & -.188 & 1 & & & & \\
\hline Drugs & .281 & .002 & .281 & 1 & & & \\
\hline Legal & $.381^{*}$ & .048 & $.345^{*}$ & $.320^{*}$ & 1 & & \\
\hline Family & .219 & .094 & $.371^{*}$ & $.356^{*}$ & .244 & 1 & \\
\hline Psychiatry & .064 & .002 & $.320 *$ & .179 & .050 & $.317^{*}$ & 1 \\
\hline
\end{tabular}

${ }^{*} p<.05$

Positive, statistically significant correlations were found between the psychosocial domains of alcohol use, legal $(r=.35)$, family $(r=.37)$, and psychiatry $(r=.32)$; drug use, legal $(r=.32)$, and family $(r=.36)$. Legal was also positively correlated with medical $(r=.38)$, and family with psychiatry $(r=.38)$.

Spirituality. As part of the revised ASI, the addition of the spiritual domain was incorporated into the ASI-NAV to specifically address AI/AN cultural issues but does not include a method of computing patient CS. The questions were categorical in nature, with some questions having an indifferent option and others merely yes or no. The number of 'yes' responses for each question are listed below in Table 13. 
Table 13

Spirituality Subscale Questions of the ASI-NAV

\begin{tabular}{|c|c|c|}
\hline Question & "Yes" Responses & $\%$ \\
\hline Do you have a belief in a "God" & 38 & $97.4 \%$ \\
\hline \multicolumn{3}{|c|}{ What changes would you like help making? } \\
\hline Learning more about prayer? & 29 & $72.5 \%$ \\
\hline Learning more about meditation? & 30 & $75.0 \%$ \\
\hline Education about a particular religion/spirituality? & 26 & $65.0 \%$ \\
\hline Changing attitude toward God/Creator? & 18 & $46.2 \%$ \\
\hline Do you have a spiritual leader or & 21 & $52.5 \%$ \\
\hline \multicolumn{3}{|l|}{ traditional/cultural person available for guidance? } \\
\hline $\begin{array}{l}\text { Do you seek out and utilize this person from time } \\
\text { to time? }\end{array}$ & 28 & $70.0 \%$ \\
\hline Are you comfortable with your spirituality and & 17 & $42.5 \%$ \\
\hline \multicolumn{3}{|l|}{ beliefs? } \\
\hline \multicolumn{3}{|c|}{ Regular Participation } \\
\hline Native American religious ceremonies/activities? & 18 & $45.0 \%$ \\
\hline Native American Church Meetings? & 13 & $32.5 \%$ \\
\hline Native American cultural activities? & 31 & $77.5 \%$ \\
\hline Native American dance activities? & 19 & $47.5 \%$ \\
\hline
\end{tabular}

\section{Cross Tabulation}

Bivariate cross tabulation was used to display the observed (i.e., count) and expected frequency for the categorical explanatory variables of employment, level of education, gender, smoking, and housing status with the dichotomous variables of mental health, substance use, and COD, listed as yes or no responses. Significant chi-square 
calculations determined which explanatory variables were included in the logistic regression models. When examining COD, all explanatory variables had frequencies within the expected range (i.e., no statistically significant findings). Tables 14-18 exhibit the statistically significant relationships for mental health and substance use disorders.

Mental health and explanatory variables. Gender, smoking status, and education did not demonstrate unusual frequency of occurrence for subjects identified as having a mental health disorder compared with those who had no documentation. Tables $14-15$ show the variation in housing and employment with membership by dichotomous mental health categories. 
Table 14.

Cross Tabulation for Mental Health Diagnosis and Housing

\begin{tabular}{|c|c|c|c|c|}
\hline & & Mental Health & Mental Health & Total \\
\hline & & No & Yes & \\
\hline \multirow[t]{2}{*}{ Rent/Own } & Count & 3 & 36 & 39 \\
\hline & Expected & 7.9 & 31.1 & 39.0 \\
\hline \multirow[t]{2}{*}{ Homeless } & Count & $8^{* *}$ & 12 & 20 \\
\hline & Expected & 4.0 & 16.0 & 20.0 \\
\hline \multirow[t]{2}{*}{ Lives with family/friends } & Count & 7 & 36 & 43 \\
\hline & Expected & 8.7 & 34.3 & 43.0 \\
\hline \multirow[t]{2}{*}{ Treatment facility } & Count & 5 & 7 & 12 \\
\hline & Expected & 2.4 & 9.6 & 12.0 \\
\hline Total & Count & 23 & 91 & 114 \\
\hline
\end{tabular}

For this cross tabulation, the cell representing homeless subjects had a higher-than expected count for ' $n o$ ' mental health diagnosis ( 8 observed vs. 4 expected; $\chi^{2}=12.501$, $p=.006$, standardized residual $=2.00$ ) yielding a statistically significantly finding. All other cells fell within an expected variation between observed and expected frequencies. 
Table 15

Cross Tabulation for Mental Health Diagnosis and Employment

\begin{tabular}{llccc}
\hline & & Mental Health & Mental Health & Total \\
& & No & Yes & \\
\hline Unemployment & Count & 21 & 49 & 70 \\
& Expected & 15.1 & 54.9 & 70.0 \\
Part-time/Disabled/Student & Count & 2 & 25 & 27 \\
& Expected & 5.8 & 21.2 & 27.0 \\
Full-time & Count & 2 & 17 & 19 \\
& Expected & 4.1 & 14.9 & 19.0 \\
Total & Count & 25 & 91 & 116 \\
\hline
\end{tabular}

$* p<.05 . * * p<.01$.

The findings showed overall statistical significance $\left(\chi^{2}=7.516, p=.023\right)$; however, none of the cells could be identified as having a significant variation from the expected frequency. As the chi-square test was performed as part of inclusion criteria for the logistic regression, this explanatory variable was incorporated into the mental health model.

Substance use disorder and explanatory variables. Chi-square testing for gender and smoking did not yield statistically significant differences between subjects with and without SUDs. Housing, employment, and education frequencies are displayed in Tables $16-18$ below. 
Table 16

Cross Tabulation for Substance Use Disorders and Housing

\begin{tabular}{llccc}
\hline & & DSM Substance & DSM Substance & Total \\
& & No & Yes & \\
\hline Rent/Own & Count & 14 & 25 & 39 \\
& Expected & 9.9 & 29.1 & 39.0 \\
Homeless & Count & 1 & 19 & 20 \\
& Expected & 5.1 & 14.9 & 20.0 \\
Live with family/friends & Count & 13 & 30 & 43 \\
& Expected & 10.9 & 32.1 & 43.0 \\
Treatment facility & Count & 1 & 11 & 12 \\
& Expected & 3.1 & 8.9 & 12.0 \\
Total & & 29 & 85 & 114 \\
\hline$* p<05 * * p<01.0$ & & & &
\end{tabular}

$* p<.05 . * * p<.01$.

The findings showed overall statistical significance $\left(\chi^{2}=9.026, p=.029\right)$; however, no independent cell could be identified as having significant variation from the expected frequency (i.e., no standardized residual value $\geq 2.00$ ). The largest discrepancy was seen in the number of homeless subjects with $n o$ SUD (observed $=1$ vs. expected $=5.1$ ). This explanatory variable was incorporated into the substance use model. 
Table 17

Cross Tabulation for Substance Use Disorders and Employment

\begin{tabular}{llccc}
\hline & & DSM Substance & DSM Substance & Total \\
& & No & Yes & \\
\hline Unemployed & Count & 10 & 60 & 70 \\
& Expected & 16.9 & 53.1 & 70 \\
Part time/ disabled/ student & Count & 10 & 17 & 27 \\
& Expected & 6.5 & 20.5 & 27 \\
Full time & Count & 8 & 11 & 19 \\
& Expected & 4.6 & 14.4 & 19 \\
Total & & 28 & 88 & 116 \\
\hline$* p<.05 . * *<.01$. & & & &
\end{tabular}

The findings showed overall statistical significance $\left(\chi^{2}=9.514, p=.009\right)$; however, no single cell could be identified as having significant variation from the expected frequency. The largest discrepancy was seen in the observed number of unemployed subjects $(n=10)$ compared to the 16.9 expected frequency, meaning fewer people were unemployed with no SUD than would be expected by chance. Also, more subjects without a SUD were employed full time ( 8 observed vs. 4.6 expected). Results for this explanatory variable were incorporated into the substance use model. 
Table 18

Cross Tabulation for Substance Use Disorders and Education

\begin{tabular}{|c|c|c|c|c|}
\hline & & DSM Substance & DSM Substance & Total \\
\hline & & No & Yes & \\
\hline \multirow[t]{2}{*}{ Less than High School Grad } & Count & $0 * * *$ & 21 & 21 \\
\hline & Expected & 4.9 & 16.1 & 21.0 \\
\hline \multirow[t]{2}{*}{ High School Grad } & Count & 8 & 36 & 44 \\
\hline & Expected & 10.3 & 33.7 & 44.0 \\
\hline \multirow[t]{2}{*}{ Some College } & Count & 12 & 27 & 39 \\
\hline & Expected & 9.2 & 29.8 & 39.0 \\
\hline \multirow[t]{2}{*}{ College Grad } & Count & $7 * * *$ & 4 & 11 \\
\hline & Expected & 2.6 & 8.4 & 11.0 \\
\hline Total & & 27 & 88 & 115 \\
\hline
\end{tabular}

The findings showed overall statistical significance $\left(\chi^{2}=18.158, p<.001\right)$; however, $25 \%$ of the cells had an expected value less than 5 . These finding suggest an inverse relationship between level of education and a SUD. A larger sample would likely yield definitive results. All subjects who earned less than a high school diploma were diagnosed with at least one SUD. Among college graduates, more subjects than expected had 'no' SUD. This explanatory variable was included in the logistic regression model because education may be associated to a SUD. 
Aim 4. To identify factors that increase the risk of substance use among AI/ANs who are seeking mental health services at an urban Indian community clinic.

Individually, possible categorical explanatory variables were tested for inclusion in logistic regression models evaluating increased risk of both a SUD and mental health disorders among AI/ANs at one clinic. No significant categorical variables were identified relating to CODs.

\section{Logistic Regression for Mental Health Disorders}

A binary logistic regression analyses was performed to predict membership in one dichotomous variable (DV), no mental health disorder was coded as ' 0 '; and presence of a mental health disorder was coded as ' 1 '. Since the dependent variable is categorical and the explanatory variables are either categorical and or continuous, the logistic regression model was used to predict membership in one of the outcome categories. Out of 100 patients in this model, there were 23 patients coded as not having a mental health disorder and 87 patients as coded having a mental health disorder. Since ASI and PHQ-9 data were limited, logistic regressions were not performed using these variables because of inadequate sample size.

Other categorical data obtained from patient's charts included employment, level of education, gender, and smoking status in this analysis. Housing was recoded into four categories: rent/own (1), homeless (2), living with family/friends (3), and treatment facilities (4). Employment was recoded into unemployed (1), part time/disabled/student (2), and full time (3). Education was recoded into four categories: less than a high school graduate (1), high school graduate (2), some college (3), and college graduate (4). Chi- 
square tests were performed on independent variables in relation to mental health disorders, with statistical significance demonstrated for housing and employment as shown in Tables 14 and 15.

Forward logistic regression was conducted to determine if employment and housing were predictors of a mental health disorder (DV). Regression results indicated a significant overall model with housing only as statistically reliable in distinguishing status of a mental health disorder (-2 Log Likelihood =100.938; Goodness of Fit =0, where observed frequencies equaled expected frequencies; $\chi^{2}(3)=11.867, p<.01$ ). The model correctly classified $79.1 \%$ of the cases. Regression coefficients are presented in Table 19. Wald statistics indicated significance in renting/owning ( $p=0.01$; odds ratio $=$ 9.167) and living with family $(p<0.05$; odds ratio $=4.26)$ when predicting the presence of a mental health disorder (Table 19).

Table 19

Logistic Regression Coefficients Related to Mental Health Disorders

\begin{tabular}{lccccr}
\hline & $B$ & Wald & $d f$ & $P$ & Odds Ratio \\
\hline Housing & & 10.890 & 3 & .012 & \\
Housing (1) & 2.216 & 6.722 & 1 & .010 & 9.167 \\
Housing (2) & .223 & .087 & 1 & .769 & 1.250 \\
Housing (3) & 1.455 & 3.942 & 1 & .047 & 4.286 \\
Constant & .182 & .091 & 1 & .763 & 1.200 \\
\hline
\end{tabular}




\section{Logistic Regression and Substance Abuse Disorders}

Binary logistic regression analyses were performed to predict membership in one dichotomous variable, no substance abuse disorder (0) or presence of a substance abuse disorder (1). Since the dependent variable is categorical and the explanatory variables are either categorical and or ordinal, the logistic regression model was used to predict membership in one of the outcome categories in this study. ASI-NAV and PHQ-9 data were limited; therefore, these logistic regressions were not performed because of inadequate sample size. Data obtained from patient's charts included employment, level of education, gender, and smoking status in this analysis. Housing was recoded into four categories: rent/own (1), homeless (2), living with family/friends (3), and treatment facilities (4). Employment was recoded into categorical variables of: unemployed (1), part time/disabled/student (2), and full time (3). Education was recoded into four ordinal categories: less than a high school graduate (1), high school graduate (2), some college (3), and college graduate (4). Chi-square tests were performed on independent variables in relation to a SUD, with statistical significance demonstrated for employment (1), education (1), and housing (3) as shown in Tables 16-18. No statistical significance was found in either gender or smoking status and SUDs.

Forward logistic regression was conducted to determine if employment, education, and housing were predictors of a substance use disorder (DV). Regression results indicated the overall model of these three predictors was statistically reliable in distinguishing status of substance abuse $(-2 \log$ Likelihood $=92.655$; Goodness of Fit $=$ $\left.1.947 ; \chi^{2}(3)=25.488, p<.001\right)$. The model correctly classified $82.1 \%$ of the cases. 
Regression coefficients are presented in Table 20. Wald statistics indicated unemployment $(1)(p=0.006$; odds ratio $=6.207)$, and education $(1)(p<0.001$; odds ratio $=.288$ ) significantly predicted substance use. For education, an inverse relationship was seen, meaning those with higher levels of education were less likely to have a SUD.

Table 20

Logistic Regression Coefficients with Substance Use Disorders as the DV

\begin{tabular}{lrcccr}
\hline & $B$ & Wald & $d f$ & $P$ & Odds Ratio \\
\hline Employment (1) & 1.826 & 7.485 & 1 & .006 & 6.207 \\
Employment (2) & .998 & 1.864 & 1 & .172 & 2.714 \\
Education & -1.246 & 12.195 & 1 & .000 & .288 \\
Constant & 3.068 & 9.402 & 1 & .002 & 21.493 \\
\hline
\end{tabular}

Note: Employment $(1)=$ unemployed; employment $(2)=$ part time/disabled/student. 


\section{Chapter 5}

\section{Conclusion}

\section{Introduction}

The purpose of this descriptive study was to gain an understanding of the prevalence of mental health disorders, ATOD, and COD among a sample of urban dwelling adult AI/ANs seeking behavioral health care at a urban AI/AN community clinic in Southwestern United States. Retrospective chart abstraction of secondary data was employed in the development of four research aims presented in this study. The findings for each individual research aim will be discussed followed by a discussion of how these findings are relative to the theoretical model, nursing practice, nursing research, nursing theory, and nursing education.

\section{Theoretical Framework}

The philosophical underpinnings of the AI/AN worldview are relational by nature with an emphasis on connectedness to the environment and other people (Hill, 2006). Traditionally, there is an emphasis on family and kinship ties that are of great importance. Native cultures generally share a collectivist orientation; needing to connect with others and the environment are essential elements in the maintenance of health, and the well being of the group is placed before the individual (Dodgson \& Struthers, 2005; Lowe, 
2002). Balance can only exist when mind, body, and spirit are working in harmonious state. If the body's emotional or spiritual elements are hurting, it will be manifested in physical forms.

According to Dapice (2006) the medicine wheel is a graphic representation of symbolic wholeness (see Figure 1). The components include four directions (spiritual, emotional, physical, and mental) that are used interchangeable in the representation of the four directions (north, south, east, and west), four seasons of the year (spring, summer, winter, and fall) and the four life givers (water, air, food, sun). These components are representative of the life cycle and pertain to universal connectedness (Poonwassie $\&$ Charter, 2001).

Social connectedness is part of the self that is subjectively aware of being close to the social world (Lee \& Robbins, 1995; 1998). The lack of social connectedness can influence cognition, perceptions and emotions, thereby affecting outward behaviors (Lee \& Robbins, 1998). A recent study by Stewart and colleagues (2009) reported that lower income persons experience a greater sense of isolation and a lower sense of belonging as compared to persons with more income. Loneliness, social support, and social connectedness are predictors of adjustment difficulties (Duru, 2008).

Cultural framework theories presented in the literature were useful in describing how principles are meaningful to an individual, how particular beliefs are relevant or worthy of attention, and which aspects that require further explanation (Bang, Medin \& Artran, 2007). Understanding how these subtle cognitive differences that affect health may lie in cultural perceptions. Americans view space as linear and time as sequential, 
whereas AI/ANs perceive space as spherical and time as cyclical. The circular concepts of cognition suggest that all points have function and significance, while linear concepts assume that some points are more significant than others (Allen, 1992). These circular concepts of thought are relative to the broad AI worldview as presented in Figure 1.

The literature supports that there are no single theories that fully explain behaviors related to risk and protective factors in the AI/AN population, but a plausible explanation can be provided in the context of historical events and perceived trauma. Social scientists have suggested that historical trauma or historical grief closely mirrored documented AI/AN health disparities, demonstrated by higher rates of prevalence for morbidity, mortality, cardiovascular disease, hypertension, alcohol abuse, depression, and suicidal behaviors (Yellow Horse Brave Heart \& Debruyn, 1998).

Whitbeck, Hoyt, and colleagues (2006) substantiated positive measures of perceived historical loss and trauma among AI elders but also confirmed its presence in younger generations of Indians. These researchers indicated that the mechanism of transmitted historical trauma is not completely understood, suggesting that more research on the affects of inter-generational trauma needs to occur. There has been a dearth of published literature indicating that AI/ANs living on reservations experience higher rates of PTSD as compared to national data (Beals et al., 2002, 2005). Theoretical concepts that describe specific indigenous factors, such as loss of culture or sense of belonging to a group, from an individual, family, and community perspective may be useful in closing the gap on AI/AN health disparities (King, Smith, \& Gracey, 2009). 
Aim \# 1- To characterize a population of AI/ANs who are seeking mental health services at an urban Indian community clinic.

Patient demographics were used to characterize AI/AN study samples through a retrospective audit of patient records. Specifically, all records for individuals that received behavioral health care services during 2008 through $2010(N=256)$ at a large urban Indian health program located in the Southwestern United States. Based upon the inclusion criteria, 123 individuals between the ages of 18 to 65 were verified as either Alaskan Native or members of a federally recognized AI tribe that comprised the sample as shown in Table 1.

Findings from this study revealed an even distribution based on gender (64 males, 59 females), with a mean age of $38.94(S D=11.01)$ years, ranging age from 18 to 65 years. There were no statistical differences in age by gender $\left(M_{\text {male }}=38.84\right.$ years; $M_{\text {female }}$ $=39.05$ years $)$. The ethnic majority of the subjects in this study was AI (95.9\%) and had completed at the minimum, a high school education (81.9\%). Since AI/ANs only comprise $1.5 \%$ of the total population, they are often disportionately represented in available studies with even fewer Alaskan Natives represented in sample data.

Education. Urban AI/ANs had the lowest percentage of subjects reporting less than a high school education (18.1\%) when compared to other studies. The highest number of subjects successfully completed high school but had no college experience (37.9\%). In this urban AI/AN sample, subjects who had less than a high school education were more likely to have a SUD than those with a college education. There were 90 urban AI/ANs identified has having a mental health diagnoses out of 115 responders, 
$34.4 \%$ had a high school education, and another $30.5 \%$ had some college education.

When comparing percentage rates of high school educational attainment with reservation AI, Whitesell et al. (2007) reported a higher dropout rate (34.1\% of subjects had less than a high school education). High school attainment rates were $35.2 \%$. Epidemiological findings from NCS data reported that $22.4 \%$ of subjects had less than a high school education and a $36.8 \%$ completed high school. These variances may be explained by metropolitan living, which lends itself to greater socio-economic opportunities for education and employment.

In this urban $\mathrm{AI} / \mathrm{AN}$ study, the lack of education appears to be a contributory factor in the presence a SUD. The percentage of subjects diagnosed with mental health disorders appeared increased as higher levels of education were attained, from $67 \%$ with less than high school to $100 \%$ for 11 college graduates; however, this difference did not attain statistical significance. A larger sample of urban AI/ANs could provide more definitive results as national research suggests that college graduates have lower rates of current illicit drug use and that higher education levels correlate with the utilization of mental health services (USDHHS, 2010b; Steele, Dewa, Lin, \& Lee, 2007).

Unemployment. Statistical data in this study indicated that unemployment was significant among urban AI/ANs reporting a SUD $(p=0.006)$. In this sample of 123 urban AU/ANs, $74(60.3 \%)$ reported being unemployed. Findings from the literature review indicated an average rate of unemployment rate of $13.6 \%$ for all AIs verses $8.6 \%$ for Caucasians in the United States (Austin, 2009). Unemployment rates in this sample 
were higher, as $50 \%$ of the reported subjects also had an AUD, while another $38 \%$ were diagnosed with MDD, and $19 \%$ of subjects reported methamphetamine dependence.

In 2010 , the average national rate of unemployment was $9.6 \%$ in the general population (U.S. Department of Labor, 2011). According to SAMHSA (USDHHS, 2010b), national rates of illicit drug use were higher among all persons who were unemployed $(17 \%)$ than among employed persons $(8 \%)$ or part-time employed $(11.5 \%)$.

Unemployment may have also been associated with the presence of a mental health disorder as 91 out of 116 subjects reported a mental health diagnoses. Among those 91 subjects, 49 were unemployed and another 25 subjects reported part time, disabled, or student employment status.

Previous ethnic studies indicated that AI/ANs with alcohol dependence had more contact with the criminal justice system (Feldstein, Venner \& May, 2006; French, 2004). This sample also yielded high rates of AUDs, and many urban AI/ANs were referred to the clinic by the criminal justice system for behavioral health treatment; thus subjects with a felony conviction or those sentenced to probation might have difficulty obtaining work or maintaining stable employment. Further studies describing the collateral negative consequences of criminal convictions for all $\mathrm{AI} / \mathrm{ANs}$ may highlight barriers to overcome and facilitate gainful employment (Akins, Smith \& Mosher, 2010; Feldstein et al.; Periman, 2007).

Housing. In the analysis of 115 urban AI responders, only $2.6 \%$ were homeowners, while $65.3 \%$ either rented or lived with family, $18.3 \%$ were homeless, $10.4 \%$ were living in a treatment facility, and $7 \%$ of the sample lived with friends. Home 
ownership appears to be higher in nationwide findings, as the U.S. Census Bureau (2009) indicated that $55 \%$ of all AVANs owned their homes. Wald statistics from logistic regression indicated a significance for renting/owning housing category $(p=0.01$; odds ratio $=9.167)$ and the other housing category of living with family $(p<0.05$; odds ratio $=$ 4.26) when predicting the presence of a mental health disorder. Further studies may be useful in establishing significant differences for housing and in relation to other variables.

Aim \#2. To examine the prevalence of mental health disorders, ATOD, and COD among AI/ANs who are seeking mental health services at an urban Indian community clinic.

Findings from chi-square analysis indicated no significant differences between genders on mental health disorders, substance use disorders, and COD $(p>.05)$. In evaluating the utilization of the clinic's behavioral health services, there were an equal number of patients who sought either mental health services or substance abuse services. Other findings in this sample identified that $56 \%$ of all of the patients had been diagnosed with a COD. National data indicated that more than 5 million persons have COD. Among samples of persons receiving substance abuse services, the rate of COD prevalence was estimated at $50 \%$ to $72 \%$ (Compton et al., 2000).

Each $D S M-I V$ code was cross-referenced to the appropriate $I C D-9$ code, as clinicians and hospitals are required to use $I C D-9$ codes for reimbursement according to the Healthcare Insurance Portability Act (HIPPA; USDHHS, 2010c). Frequencies for the $D S M-I V$ diagnoses and $I C D-9$ codes were examined to measure differences between 
variables for 122 subjects (range $=1$ to 8 diagnostic codes). For subjects diagnosed in DSM-IV categories, $79 \%$ had a mood disorder, $62 \%$ were categorized with an AUD, $60 \%$ had other substance-related disorders, and $30 \%$ of subjects had an anxiety-related disorder.

In a comparative study $(N=861)$, AI caretakers of AI children reported alcohol abuse rates of $63.6 \%$, drug abuse rates of $20 \%$, MDD in $19.2 \%$, and $5.5 \%$ with a generalized anxiety disorder (Whitbeck, Johnson et al., 2006). Findings from this larger AI sample of caretakers applied the same DSM-IV categories and reported similar diagnostic frequencies as compared to this urban AI/AN sample.

Results from this study included the highest frequencies for the most commonly applied ICD-9 codes. Alcohol dependence was coded as ICD-9 303.9 and reported in $50 \%$ of the cases. The second most frequently reported ICD-9 code was 296.32 , indicating a major depressive disorder in $38 \%$ of the subjects. Methamphetamine and psycho-stimulant dependence was ranked as the third highest frequency and translated into ICD-9 code 304.4 among $19 \%$ of the subjects in this study. Conversely, the NSDUH published conflicting data suggesting that AI/ANs living in metropolitan areas during 2004-2005 were less likely to use illicit drugs than their counterparts not living in metropolitan areas (USDHHS, 2007c).

Alcohol. In this sample, there was a significant prevalence rate of $78.9 \%$ for alcohol abuse, with $26 \%$ of subjects reporting five or more daily drinks. In a study of 754 urban AI/AN by Shore and colleagues (2002), lifetime alcohol abuse rates were $56 \%$ and current alcohol abuse rates were 27\%. Current NSDUH rates for past month alcohol use 
among AV/AN were found to be lower than the national average (43.9\% verses $55.2 \%$ ), however for past month binge drinking they were $14 \%$ higher as compared to the general population (USDHHS, 2010d). Other national data identified that $52 \%$ of all adults in the United States are current regular drinkers, defined as 12 or more drinks in the past year (USDHHS, 2010b). In 2001-2002, epidemiological studies such as NESARC reported that past year alcohol use rates were higher among Caucasians at $74.3 \%$ verses past year alcohol rates for AI/AN of $65.5 \%$ which were significantly lower by comparison (Falk, Hsiao-ye, \& Hiller-Sturmhöfel, 2008).

The NSDUH is an excellent source of data on SA in ethnic minorities, but somewhat limited as it does not employ multivariate analysis in published results. In light of the current findings, future racial studies may be useful to delineate or measure the varying affects of exposure to risk factors over one's lifespan.

Tobacco. Prevalence rates were $44 \%$ for current smokers in this sample and were similar to nationwide data, which estimated a $41 \%$ prevalence rate for all $\mathrm{AJ} / \mathrm{AN}$ smokers (Grim, 2007). Findings from this study indicated that patients who had reportedly quit smoking were significantly older than current smokers $\left(M_{\text {Quit }}=43.0\right.$ years vs. $M_{\text {Smoke }}=$ 35.3 years, $F[2,107]=4.577, p=.012$ ), but neither group was significantly different from non-smokers $(M=39.5$ years $)$. Patients who reportedly quit smoking and subjects with no smoking history had significantly higher levels of education (median = some college) than those who were active smokers (median = high school graduates; $F[2,103]$ $=5.972, p=.004)$. Findings from chi-square analysis indicated no significant difference in smoking status between genders $\left(\chi^{2}=1.501, p>.05\right)$. 
National data indicated that young AI/ANs had the highest smoking prevalence rate $(24 \%)$, but smoked fewer cigarettes on a daily basis (CDC, 2006). According to the CDC (2008c), smoking rates are highest among Whites and AI/ANs. More studies are needed to generate support for programs aimed at reducing rates of tobacco dependence for this ethnic group.

Other illicit drug use. Prevalence rates for illicit drug use were estimated for 123 subjects in this sample. Aside from the previously reported high rates of AUD, there was also a $52 \%$ prevalence rate for cannabis use and a $45 \%$ prevalence rate for methamphetamines. Among all subjects, there was a 35\% prevalence rate for polysubstance abuse. There were no significant differences in crosstabs for gender and substance abuse with the exception of age at first use (see Aim 4). There were slightly more males than females using amphetamines and cannabis in this sample.

In 2009, the NSDUH reported that AI/ANs had the highest rate for substance abuse (15.5\%) as compared to other ethnic groups (USDHHS, 2010b). Findings from the NCS indicated that AI men had higher rates of cannabis use than other ethnic groups (Stinson, Ruan et al., 2006). Other national data from NESARC reported higher rates of co-use among AI/ANs as compared to other races (Falk et al., 2008). Higher rates of reported illicit drug use identified in this sample could be attributed to the fact that all subjects were specifically seeking behavioral health related services at the clinic.

Depression. Findings revealed that there were more documented $I C D-9$ codes for depression than available PHQ-9 screenings. A statistical expression was generated for concordance of $I C D-9$ depression codes and scores from PHQ-9 to survey its accuracy in 
diagnosing depression, thus a two-by-two table was constructed to compare positive and negative test results with the diagnostic codes in the chart. Sensitivity measured the agreement between a positive test result (e.g., MDD) and a diagnostic code for depression (sensitivity $=64.29 \%$ ). Specificity measures the ability of the test to yield a negative result when, in fact, no diagnostic code is warranted (specificity $=72.22 \%$ ).

For those subjects who had a completed PHQ-9 $(n=46)$, the prevalence rate for depression was $60.9 \%$. Among the larger group of 122 subjects, the prevalence rate for those with diagnostic depression codes was $64.8 \%$. Using these data, the positive predictive value was calculated at $78 \%$ and negative predictive value at $57 \%$.

Psychiatric disorders among two large AI reservations were compared to nationwide findings from NCS prevalence rates. Findings between the two AI reservations revealed higher rates of poverty, depressive/anxiety disorder, and PTSD (Beals et al, 2005). In contrast to the NCS findings and supported by several other studies, 12-month prevalence rates for MDE among subjects between the ages of 18 to 25 was highest among persons reporting origins of two or more races, followed by Native Hawaiian/Pacific Islanders and AV/ANs (USDHHS, 2007c).

Findings from national studies, including the NESARC survey $(N=43,093)$, reported on rates for depressive disorders and found that AIs had the highest lifetime prevalence of MDD (19.17\%) when compared with other ethnic groups (Hasin, et al., 2005). Among elderly AIs, prevalence rates for depression were 32\% (Manson, 2000). A small study by Duran and colleagues (2004) identified that the lifetime prevalence for all documented mood disorders in primary care was $44 \%$ ( $n=234$ AI women). 
Additionally, $81 \%$ of the AI women who reported a mood disorder also indicated concurrent major depression.

Conclusive findings from the review of literature supported that depression was more likely to occur with progressive substance abuse and that chronic substance abuse might be a contributing factor in the development of depressive disorders (Sinha \& Rounsaville, 2002). The data from this study produced similar findings, as a significant number of subjects reported a SUD and $50 \%$ of the subjects also had a simultaneous depressive disorder.

Gender. There was no difference between genders and any PHQ-9 questions, with the exception of poor appetite or overeating where women scored significantly lower (Mann Whitney $U=170.00, p<.05$ ). There was no statistically significant correlation between the PHQ-9 total score and age $(r=.172, p>.05)$. Interestingly, this finding was not supported in the general literature, as women reported higher prevalence rates of somatic depression symptoms, such as difficulty sleeping or loss of appetite (Silverstein, 2002).

Results from $t$-tests revealed significant differences between gender and age at first use as follows: alcohol $(t[96]=-2.321, p=.022)$, methadone $(t[5]=-3.842$, $p=.018)$, and cannabis $(t[63]=-2.053, p=.044)$. In all cases with significant differences, males began their substance abuse at an earlier age when compared to women in this study. Men reported substance use at an earlier age among all categories, with the exception of inhalants. Six people reported inhalant use. A $t$-test for significance could not be performed because only one woman reported using inhalants. 


\section{Thirty Day and Reported Years of Substance Use}

ASI-NAV interview subjects were asked to report the number of days of alcohol and drug use for the past 30 days and the number of years of regular use. Two alcohol questions are designed to capture the frequency of any drinking and binge drinking. The substance abuse assessment included information on eleven most commonly abused substances. In this study, the most frequently abused substances in order were alcohol, cannabis, and cocaine.

\section{Thirty Day and Lifetime Reports of Violence}

Study results indicated that 17 of 82 subjects reported problems controlling violent behavior within the last 30 days. Of those, $15(88 \%)$ were also diagnosed with a SUD. Seventy-seven percent of subjects experienced problems controlling violent behavior at some point in their lifetime in this study, while in a comparative study of AI women $(N=312)$ reported lifetime physical or sexual victimization rates of $59 \%$ (Malcoe, Duran, \& Montgomery, 2004). Tehee \& Esqueda (2008) found that American Indian and European women have different internal conceptualizations of what domestic violence is and causal events preceding acts of violence.

Other notable results from this study indicated that approximately one in four subjects reported at least one suicide attempt (interval violence). In 2007, suicide among AVANs was the second leading cause of death for ages 15-34 years old. In the general population, suicide was the eleventh leading cause of death and one-third of all persons committing suicide were under the influence of alcohol at the time of their death (CDC, 2009b). 
Aim \#3 To identify factors that contribute to mental health disorders, ATOD, and COD among AI/ANs who are seeking mental health services at an urban Indian community clinic.

The revised ASI-NAV was administered to only 43 subjects receiving behavioral health care services over the preceding 3-year period (35.0\%). Two scores were collected from revised ASI-NAV: composite scores (CS) and the interviewer's score. McLellan et al. (1985) acknowledged that the number of specific items comprising total ASI CSs might not be equal; however, each of the seven domains were weighted equally to ensure internal validity and consistency with Cronbach's alpha set at 0.70 .

In this study, ASI-NAV CS were obtained for the eight domains representing psychosocial functioning as defined by medical, employment, alcohol use, drug use, legal, family, psychiatry, and spiritual. Subjects coded with a mental health $I C D-9$ code had significantly higher CS in the psychosocial functioning area of psychiatry (0.597 vs. $0.329, t=-2.720, p=.01)$ than those who did not have a mental health $I C D-9$ code.

AI/ANs who were identified as having a co-morbid SUD and a mental health diagnoses by ICD-9 codes also had significantly higher psychiatry CS on the ASI-NAV, than people who did not have co-morbidity ( 0.519 vs. $0.327, t=-2.962, p<.01)$. There were no other significant differences in the remaining six ASI-NAV CS domains with regard to mental health disorders or co-morbid diagnoses.

\section{Interviewer's Rating of Need for Treatment by ASI-NAV CS}

In recognizing cultural and gender differences in chemically dependent patients, the interviewer's perception of the severity of a SUD may differ from the patient. During 
the substance abuse assessment at the clinic, the interviewer rated the need for counseling in each of the eight ASI-NAV CS categories/domains. Severity profiles for CSs ranged from 0 (no need for counseling) to 9 (most severe need for counseling). A comparison by gender yielded no significant differences ( $t$-test range from -0.396 to $1.156, p>.05$ ); therefore, the interviewer rating of severity was not significantly different by gender. Wertz, Cleaveland, and Stephens (1995) identified that ASI interviewers in the clinical setting had less agreement on subjective judgments for problem severity in each domain and noted a low inter-rater correlation for the alcohol severity subjective measure. Alcohol CS were the highest among the severity ratings and alcohol was reported as the drug of choice among subjects in this study; however, the validity of this subjective measure in the clinical setting should be used with caution. Several researchers noted that the ASI was less reliable in patients with SMI or persistent mental health disorders, as interviewers identified that $31 \%$ of the subjects misunderstood the intent of the questions (Zanis, McLellan, \& Corse, 1997). These findings suggest that further studies are necessary to verify that clinicians can achieve inter-rater reliability comparable to researchers skilled in survey administration as reported by author of the ASI tool (McLellan et al., 1985)

\section{Patient's Rating of Need for Treatment by ASI-NAV CS}

During the substance abuse assessments, patients were asked to rate their perceived severity of the problem and rate their need for treatment on the ASI-NAV in each of the domains. Contrasting the interviewer's severity ratings on a 0 to 9 scale, patients scored their severity from 0 (minimal rating) to 4 (severe rating). Results 
indicated that patients perceived their need for treatment higher than the severity of the domain, with the exception of employment and medical, only the need for alcohol treatment was statistically higher than the severity of the problem (Wilcoxon Signed Rank Test, $z=-2.032, p<.05$ ). In a retrospective study by Evans, Spear, Huang \& Hser (2006), records matched for urban AI and non-Indian persons ( $n=368$ each group) indicated similar high rates for alcohol severity CS on the ASI and yielded similar levels of improvement with SA treatment. Conclusive results indicated that AI had fewer treatment sessions, an increased number of residential stays for SA, and fewer post treatment arrests as compared to non-Indian subjects. A notable limitation in this study was that the ASI-NAV was administered only once during initial subject SA assessments; therefore, predictive analysis on the effectiveness of SA treatment using post treatment ASI CSs may have been useful in supporting outcome measures.

\section{Correlation of ASI-NAV Composite Scores}

Of the 43 subjects with completed ASI-NAV scores, significant correlations related to either drug or alcohol abuse and legal problems. This finding was not unexpected as $44 \%$ of ASI-NAV completers reported they were on probation or parole. Family issues also correlated to either drug or alcohol abuse. Only alcohol abuse (not drug abuse) was positively correlated to psychiatric composite scores. Interestingly, composite drug scores were not significantly correlated to composite alcohol scores, despite $57 \%$ of subjects having documented CODs. One possible explanation was the relative low number of ASI completers. In a small retrospective study of $(N=31)$, ASI composite scores were verified for agreement with patient charts and accurately classified 
low and high severity scores for SA (Quercioli, Messina, Fini, Frola, \& Nante, 2010). Research on a larger population of subjects is warranted to address gaps in the literature.

\section{Correlation of ASI-NAV Scores and Spirituality}

The results of the Spirituality section of the ASI-NAV indicated a dissonance between belief in "God" (97\%) and comfort with that belief (43\%). Most ASI-NAV completers expressed an interest in learning more about prayer and meditation. The spirituality section was added to ASI-NAV to recognize this essential dimension of AI culture, but its usefulness as a diagnostic tool is limited. Currently, no scoring mechanism or interpretative guidance has been forthcoming in the literature.

Aim \#4 To identify factors that increase the risk of substance use among AI/ANs who are seeking mental health services at an urban Indian community clinic.

In a cross tabulation between employment and SUDs $(n=116)$, this study revealed 62 subject $(53.2 \%)$ were unemployed and another $24(20.5 \%)$ maintained parttime, disabled, student status in the recoded categorical data for employment. Robins \& Reiger (1991) reported that persons with SMI have longer periods of unemployment and their income tends to be significantly less as compared with the U.S. population. In the general population, unemployment rates were $17 \%$ among adults using illicit drugs verses $8 \%$ for persons who were employed full-time (USDHHS, 2010b). According to Austin (2009) unemployment rates for AI were highest in the Midwest at $19.3 \%$ and were $21.3 \%$ among $\mathrm{AN}$, and notably lower for whites with a rate of $9.1 \%$.

Logistic regression. Data analysis of Wald statistics from logistic regression indicated a significance for renting/owning housing category $(p=0.01$; odds ratio $=$ 
9.167) and the other housing category of living with family $(p<0.05$; odds ratio $=4.26)$ when predicting the presence of a mental health disorder. The lack of evidence associating homelessness with mental health disorder could be due to the transitional nature of their circumstances, thus preventing repeat clinic visits for a comprehensive behavioral health assessment. Further studies may be useful in establishing significant differences for housing and other variables.

In analyzing the explanatory factors associated to substance abuse, logistic regression detected a significant inverse relationship related to the level of education among subjects and being unemployed. Results from this study indicated that higher levels of education implied the lower likelihood of a SUD. The only other predictive variable associated with a SUD was being unemployed.

In 2009, national data suggested that education levels and overall illicit drug use appeared to increase from 2008 to 2009 (8.1\% verses $10.2 \%)$, and that college graduates had lower rates of illicit drug use as compared to persons who did not graduate high school $(6.1 \%$ verses $10.2 \%)$ but cited that college graduates were more likely to have tried illicit drugs during their lifetime. According to the NSDUH report of 2009, past month binge alcohol use and illicit drug use were higher among AI/ANs than the national average rate of $30.6 \%$ verses $24.5 \%$, and $11.2 \%$ verses $7.9 \%$ (USDDHS, 2010b).

\section{Significance of the Study}

The significance of this study rested in the development of appropriate research aims that captured accurate data in describing the characteristics of a growing urban AI/AN population with substance abuse and mental health disorders with few available 
studies. Findings from this study are presented to inform researchers in the development of evidenced based treatment programs that may be culturally appropriate for urban AJ/ANs. A search of PubMed using the terms of, urban "American Indian" and mental health, yielded merely 33 citations. In general, there were few descriptive or comparative studies addressing the prevalence of mental health disorders, COD, and ATOD among urban AI/ANs, therefore, findings from this descriptive study may be useful in addressing specific mental health disparities that impact urban AI/ANs.

\section{Implications for Nursing Practice}

A large body of literature supports the concept that AVANs do not perceive mental health as separate from spiritual or physical health, therefore mental health providers and nurses must incorporate the indigenous worldview concept while providing culturally integrated care within this unique population (Grandbois, 2005; Hill, 2006; Struthers \& Lowe, 2003). Measurable outcomes are likely to improve if health promotion activities are delivered in ways that are consistent with the norms and values held by AI/ANs.

\section{Implications for Nursing Research}

A public health priority in most Indian communities is to close the gap on persistent health disparities. Research in AI/AN communities may differ from mainstream when issues arise while negotiating between political entities of tribal governments, Indian Health Service, academia and nursing science (Holkup et al., 2009). $\mathrm{AI} / \mathrm{AN}$ nurses are in a favorable position to deliver health care to Indian people, however 
continue to remain under-represented as nurse scientists among research focused on health disparities (Henly et al., 2006).

Plausible solutions for health disparities may be explained though translational health research, which suggests that basic medical research should apply real-world applications through interdisciplinary science that indirectly or directly affect people's health (Unger, Soto \& Thomas, 2008). These researchers explored and applied translational research strategies in AI communities by suggesting the following; incorporating and recognizing the role of wisdom from elders by involving them in the planning and implementation phases of research; use of communication that is culturally appropriate, as AIs prefer to communicate in person rather than using electronic media; including elements of spirituality or prayer; recognizing aspects of unresolved grief/historical trauma; and recognizing the roots of AI distrust of outsiders who engage in helicopter research activities that rarely lead to sustainable health programs after the research has been completed in the Indian community (Grim, 2006; \& Unger et al. 2008).

\section{Recommendations for Nursing Theory}

Theoretical concepts that describe specific indigenous factors, such as loss of culture or sense of belonging to a group, from an individual, family, and community perspective may be useful in closing the gap on AI/AN health disparities (Hill, 2006; King, Smith, \& Gracey, 2009). Research studies have produced valid and reliable measures indicating the presence of intergenerational and historical trauma by using the Historical Loss scale (HTS) and the Historical Loss Symptoms Scale, however further 
studies are warranted in identifying other possible contributing factors (Whitbeck, Adams, Hoyt, \& Chen, 2004).

Unger and colleagues (2008) assert that AI/AN collectivist subgroups may be considered high risk in the context of living within a larger individualistic culture, as their cultural values are not often honored or respected by larger society. Larger societies then define the criteria for success via high levels of education and income. The concerns of the collectivist subgroups are then ignored by imposing individualistic goals of the larger society who created the criteria thereby, limiting opportunities for success for those belonging to the collectivist subgroup.

\section{Recommendations for Nursing Education}

A major step in addressing health care disparities is to increase access to mental health services. The passing of the Mental Health Parity and Addiction Equity Act of 2008, now ensures equal reimbursement for mental health care services in alignment with other medical or surgical billable services (USDHHS, 2010a). By 2025, studies are projecting a large physician shortage in rural and some urban areas (Kane et al., 2009).

Advance practice nurses are in the unique position of filling the provider gap and adding to the science of mental health and health disparities. The development of culturally appropriate preventative efforts or effective treatment modalities is a priority. Findings from this study may support existing interventions or indicate the need for tailoring treatments aimed at reducing the morbidity and mortality among urban dwelling AI/ANs with substance abuse and mental health disorders. 
The lack of diversity in nursing may create barriers in the delivery of culturally competent patient care for AI/ANs. In 2009, the National League of Nursing (NLN) recognized the need for global diversity within the nursing profession by citing that only $0.6 \%$ of all AV/ANs hold nurse educator positions and $0.3 \%$ of all professors were AI/AN. The Robert Wood Johnson Foundation (RWJF; 2010) has also supported efforts to address the global nursing shortage by concluding that collaborative efforts and effective strategies are necessary to recruit and retain $\mathrm{AI} / \mathrm{AN}$ students into nursing programs. The success of AI/AN nursing students may be enhanced by opportunities that involve faculty mentoring, however more studies are needed to evaluate the effectiveness of mentoring relationships and student success.

\section{Ethical Considerations}

Culturally competent researchers should apply indigenous values of respect, cooperation, involvement in the process, and guided exchange of ideas in research practice (Sobek, Chapleski, \& Fisher, 2003). Research studies indicate that AI communities have reported negative interactions with outside researchers, as the priority of the researcher often conflicts with the needs of the Indian community in developing sustainable programs which improve the overall health of its tribal members (Poupart, Baker \& Red Horse, 2009). Davis and Reid (1999) identified several research studies that were historically harmful to AI communities, such as the epidemiological study on an outbreak of syphilis that violated patient privacy when neighboring communities became aware of the situation, the lack of consent from the Navajo tribe after receiving requests from the tribe for non-disclosure of published reports from 1993-1994 related to 
the hantavirus pulmonary syndrome on their reservation, and an alcoholism study that led to adverse credit ratings of tribal members by banking lenders.

Recently, the New York Times reported that the Havasupai tribe settled a multimillion dollar lawsuit involving blood samples taken for a diabetic study but was later used for other genetic studies on mental illness, and to dispute the tribe's story regarding their geographical origins without informed consent from tribal members (Harmon, 2010). These ethical considerations were breached, resulted in harm, and created a distrust of outsiders seeking to perform research in Indian communities, thus researchers must consider cultural influences that influence patient communication and listening when obtaining informed consent for future studies.

Today, tribal programs are aware of the policies and need for outcome research that often generates continued federal funding. Several studies have successfully relied on participatory models of research that included input from the community prior to the commencement of any research activities. These creative partnerships yield beneficial outcomes for both the researcher and community targeted for study (Poupart et al., 1999).

\section{Limitations of the Study}

Several limitations of the study included the use of pre-collected data, and inconsistent, inaccurate, or missing data that resulted in a smaller sample size than originally anticipated. Although every attempt was made to find the first documented incidence of mental health, substance abuse, and co-occurring mental health issues, there was no contact with patients or nursing staff, only the documented data in the medical record was available for recording of variables. There may have been inconsistencies in 
coding for psychiatric diagnoses or ICD-9 codes related to the level of education and experience of the provider e.g. marital family therapist verses clinical psychologist.

Random sampling reduces bias in studies and comparison groups were not employed in this study. The sample size was small due to the number of available AI/AN subjects in this study. Caution is warranted in small studies that apply logistic regression, as small or moderate size samples may overestimate the effect measure when overestimated effect sizes are pooled together (Nemes, Jonasson, Genell, \& Steineck, 2009).

\section{Conclusions}

National health disparities data for the entire AI/AN population has been useful in identifying specific health problems for all Indians but may exclude other explanatory factors that impact urban AV/ANs. Since $60-67 \%$ of the total indigenous population has been relocated through governmental policies or "urbanized," more local or community health disparities research is needed to highlight other distinct physical, socioeconomic, or mental health factors that contribute to poorer health among urban Indians (CDC, 2003c; UIHC, 2007).

Findings from this study were useful in providing a greater understanding of the prevalence of mental health disorders, ATOD, and COD among a sample of urban dwelling adult AI/ANs seeking behavioral health care at an urban AI/AN community clinic in Southwestern United States. Qualitative methods may be useful in exploring norms and perceptions of AV/ANs regarding substance use, as research indicates that an individual's personal view influences behaviors that determine drug use (Gold \& 
Nguyen, 2009). Review of the literature indicated that AI/ANs have high rates of alcohol and marijuana use, and similar findings were noted in this study. Since there are persistent gaps in the literature that address urban AI/AN health disparities, larger studies are necessary to delineate and explain specific factors that contribute to psychiatric and substance use disorders.

Factors related to childhood adversities and the development of adult psychopathology were not explored and may provide insight to the link between living with family and having a diagnosed mental health disorder as noted in this study. Other findings from this study suggested that having a higher education implied a lower likelihood for the presence of a substance use disorder, indicating the need for higher educational programs that are accessible to AIANs.

Approximately $70 \%$ of subjects in this study sought out persons for spiritual guidance, while $53 \%$ of the subjects actually had a traditional or cultural person they relied on for guidance. Elders have traditionally played a role in the dissemination of cultural knowledge and the sharing of values among AI/AN groups, however there were few available studies that described the affects of urbanization, sense of belonging, loss of culture, and the sense of connectedness when a substance use disorder or mental health diagnoses was present.

$\mathrm{AI} /$ tribes have the shared belief that spirituality is interconnected to health and well-being (Limb \& Hodge, 2008). Addressing health disparities from a holistic perspective may lead to improved outcomes. Further studies of contributing factors that 
lead to persistent health disparities are warranted to improve the physical and mental health status for all American Indian and Alaska Native people. 


\section{References}

Agency for Health Care Research and Quality. (2008). 2007 national healthcare quality and disparities report. (AHRQ Publication No.08-0041). Washington, DC: U.S. Department of Health and Human Services.

Akins, S., Smith, C. L., \& Mosher, C. (2010). Pathways to adult alcohol abuse across racial/ethnic groups: An application of general strain and social learning application of general strain and social learning theories. Journal of Drug Issues, 40(2), 321 351.

Alaska Department of Health and Social Services, Tobacco Prevention and Control Program (2007). Alaska Native pregnant women. Retrieved from http://www.hss.state.ak.us/dph/chronic/tobacco/PDF/pregnancy_fact_sheet.pdf

Allen, P. G. (1992). The sacred hoop: Recovering the feminine in American Indian. Boston, MA: Beacon Press.

American Psychiatric Association. (1987). Diagnostic and Statistical Manual of Mental Disorders (DSM-III-R: Revised 3rd ed.). Washington, DC: Author.

American Psychiatric Association. (1994). Diagnostic and statistical manual of mental disorders (DSM-IV, 4th ed.). Washington, DC: Author.

Andrews, G., Hall, W., Teesson, M., \& Henderson, A. S. (1999). The mental health of Australians. Canberra: Commonwealth Department of Health and Aged Care.

Aud, S., Fox, M., \& Kewal-Ramani, A. (2010). Status and trends in education of racial and ethnic groups (NCES 2010-015). U.S. Department of Education, National Center for Education Statistics. Washington, DC: Government Printing Office. 
Austin, A. (2009). American Indians and the great recession: Economic disparities growing larger (Issue Brief No. 264). Washington, DC: Economic Policy Institute. Retrieved from http://epi.3cdn.net/1aaad254862c0b29b6_2em6bnkyh.pdf

Bang, M., Medin, D. L., \& Atran, S. (2007). Cultural mosaics and mental models of nature. Proceedings of the National Academy of Sciences, 104, 13868-13874.

Bar-On, D., Eland, J., Kleber, R. J., Krell, R., Moore, Y., Sagi, A., . . van IJzendoorn, M. H. (1998). Multigenerational perspectives on coping with the holocaust experience: An attachment perspective for understanding the developmental sequelae of trauma across generations. International Journal of Behavioral Development, 22(2), 315338.

Beals, J., Manson, S. M., Shore, J. H., Friedman, M., Ashcraft, M., Fairbank, J., \& Schlenger, W. (2002). The prevalence of post-traumatic stress disorder among American Indian Vietnam veterans: Disparities and context. Journal of Traumatic Stress, 15, 89-97.

Beals, J., Manson, S. M., Mitchell, C. M., Spicer, P., \& The Al-SUPERPFP Team. (2003). Cultural specificity and comparison in psychiatric epidemiology: Walking the tightrope in American Indian research. Culture, Medicine \& Psychiatry, 2, 259-89.

Beals, J., Novins, D. K., Whitesell, N. R., Spicer, P., Mitchell, C. M., \& Manson, S. M. (2005). Prevalence of mental disorders and utilization of mental health services in two American Indian Reservation populations: Mental health disparities in a national context. American Journal of Psychiatry, 162(9), 1723-1732. 
Beauvais, F. (1992). Comparison of drug use rates for reservation Indian, non-reservation Indian and Anglo youth. American Indian and Alaska Native Mental Health Research, 5, 13-31.

Beauvais, F. (1998). American Indians and alcohol. Alcohol Health \& Research World, $22(4), 253$.

Beery, W. L., Schoenbach, V. J., Wagner, E. H., Graham, R., Karon, J., \& Pezzullo, S. (1986). Health risk appraisal: Methods and programs with annotated bibliography (Department of Human Health and Services Publication No. PHS 86-3396). National Center for Health Services Research and Health Care Technology: Washington, DC: Government Printing Office.

Bennett, T. (1992). Unpublished findings from a study of California Indian health needs, report mandated by Congress: Public Law 100-713. San Francisco, CA: University of California.

Berry, J. W., Kim, U., Minde, T., \& Mok, D. (1987). Comparative studies of acculturative stress. International Migration Review, 21(3), 491-511.

Bourdan, K. H., Rae, D. S., Locke, B. Z., Narrow, W. E., \& Reiger, D. A. (1992). Estimating the prevalence of mental disorders in U.S. adults from the Epidemiologic Catchment Area Survey. Public Health Reports, 107(6), 663-68.

Burns, L., \& Teesson, M. (2002). Alcohol use disorders comorbid with anxiety, depression and drug use disorders: Findings from the Australian National Survey of Mental Health and Well Being. Drug and Alcohol Dependence, 68(3), 299307. doi: 10.1016/S0376-8716(02)00220-X 
Carise D., \& McLellan A. T. (1999). Increasing cultural sensitivity of the Addiction Severity Index (ASI): An example with Native Americans in North Dakota. Substance Abuse and Mental Health Services Administration (SAMHSA), Center for Substance Abuse Treatment.

Castor, M. L., Smyser, M. S., Taualii, M. M., Park, A. N., Lawson, S. A., \& Forquera, R. A. (2006). A nationwide population-based study identifying health disparities between American Indians/Alaska Natives and the general populations living in select urban counties. American Journal of Public Health, 96(8), 1478-1484.

Centers for Disease Control \& Prevention. (1998). Smoking \& tobacco use: Highlights: American Indians and Alaska Natives and tobacco. Retrieved from http://www.cdc.gov/tobacco/data_statistics/sgr/1998/highlights/am_indian_alaska_ native/

Centers for Disease Control \& Prevention. (2002a). Census 2000 briefs and special reports series. Retrieved from http://www.census.gov/population/www/cen2000/briefs.html.

Centers for Disease Control \& Prevention. (2002b). Fetal alcohol syndrome - Alaska, Arizona, Colorado, \& New York, 1995-1997. $M M W R$, 51(20), 433-425. Retrieved from www.cdc.gov/mmwr/preview/mmwrhtml/mm5120a2.htm

Centers for Disease Control \& Prevention. (2003a). Health disparities experienced by American Indian and Alaska Natives. MMWR, 52(30), 697-724. Retrieved from http://www.cdc.gov/mmwr/PDF/wk/mm5230.pdf 
Centers for Disease Control \& Prevention. (2003b). 10 leading causes of death, United States 2003, American Indian/Alaska Native, both sexes. Retrieved from http://www.cdc.gov/injury/index.html

Centers for Disease Control \& Prevention. (2003c). Health status of American Indians compared to other racial/ethnic minority populations-selected states 2001-2002. $M M W R, 203(47), 1148-1152$. Retrieved on May 1, 2006, from http://www.cdc.gov/mmwr/preview/mmwrhtml/mm5247a3.htm

Centers for Disease Control \& Prevention. (2006). Racial/ethnic differences among youths in cigarette smoking and susceptibility to start smoking- United States 2002-2004. MMWR, 55(47), 1275-77.

Centers for Disease Control \& Prevention. (2008a). Alcohol-attributable deaths and years of potential life lost among American Indians and Alaska Natives - United States, 2001 - 2005. $M M W R, 57,938-941$.

Centers for Disease Control \& Prevention. (2008b). Fact sheet: HIV/AIDS among American Indians and Alaska Natives. Retrieved from http://www.cdc.gov/hiv/resources/Factsheets/PDF/aian.pdf

Centers for Disease Control \& Prevention. (2008c). Targeting tobacco use: The nation's leading cause of preventable death at a glance. Retrieved from http://www.cdc.gov/print.do?url=http://www/cdc/gov/NCCDPHP/publications/aa g/osh.htm.

Centers for Disease Control \& Prevention. (2009a). Prenatal smoking data book: Introduction. Retrieved from http://www.cdc.gov/reproductivehealth/prenatalsmkbk/introduction.htm 
Centers for Disease Control \& Prevention (2009b). Suicide: Facts at a glance. Retrieved from http://www.cdc.gov/violenceprevention/pdf/Suicide_DataSheet-a.pdf Churchill, W. (1997). A little matter of genocide: Holocaust and denial in the Americas, 1492 to the present. San Francisco, CA: City Light Books.

Cohen, J. (1988). Statistical power analysis for the behavioral sciences (2nd ed.). Philadelphia, PA: Lawrence Erlbaum \& Assoc.

Collins, R. L., \& McNair, L. D. (2002). Minority women and alcohol use. Alcohol Research \& Health, 26(4), 251-255.

Compton Ill, W. M., Cottler, L. B., Abdallah, A. B., Phelps, D. L., Spitznagel, E. L., \& Horton, J. C. (2000). Substance dependence and other psychiatric disorders among drug dependent subjects: Race and gender correlates. American Journal on Addictions, 9(2), 113-125.

Dapice, A. (2006). The medicine wheel. Journal of Transcultural Nursing, 17, 251-260.

Davis, S. M., \& Reid, R. (1999). Practicing participatory research in American Indian communities. American Journal of Clinical Nutrition, 69(4), 7555-7595.

Demidenko, E. (2006). Criteria for global minimum of sum of squares in nonlinear regression. Computational Statistics \& Data Analysis, 51(3), 1739-1753. doi:10.1016/j.csda.2006.06.015

Denny, C. H., Holtzman, D., \& Cobb, N. (2003). Surveillance for health behaviors of American Indians and Alaska Natives: Findings from the Behavioral Risk Factor Surveillance System, 1997-2000. MMWR Surveillance Summaries, 52(SS07), 113. 
Dhalia, S. \& Kopec, J. A. (2007). The CAGE questionnaire for alcohol misuse: A review of reliability and validity studies. Clinical Investigative Medicine, 30(1), 33-41.

Dodgson, J. E., \& Struthers, R. (2005). Indigenous women's voices: Marginalization and health. Journal of Transcultural Nursing, 16(4), 339-346.

Doka, K. (1987). Silent sorrow: Grief and the loss of a significant other. Death Studies, $11,441-449$.

Doka, K. J. (1989). Disenfranchised grief: Recognizing hidden sorrow. Lexington, MA: Lexington Books.

Donohue, J. M., \& Frank, R. G. (2007). Estimating Medicare Part D impact on medication access among dually eligible beneficiaries with mental disorders. Psychiatric Services, 58(10), 1285-1291.

Duran, B., Duran, E., \& Yellow Horse Brave Heart, M. (1998). Native Americans and the trauma of history. In R. Thornton (Ed.), Studying Native America: Problems and prospects (pp. 60-78). Madison, WI: University of Wisconsin Press.

Duran, B., Sanders, M., Skipper, B., Waitzkin, H., Malcoe, L., Paine, S., \& Yager, J. (2004). Prevalence and correlates of mental disorders among Native American women in primary care. American Journal of Public Health, 94(1), 71-77.

Duran, E., \& Duran, B. (1995). Native American postcolonial psychology. Albany, NY: SUNY Press.

Duru, E. (2008). The predictive analysis of adjustment difficulties from loneliness, social support and social connectedness. Educational Sciences: Theory and Practice, $8(3), 849-856$. 
Earle, K. A. (2000). Child abuse and neglect: An examination of American Indian data. Portland, OR: National Indian Child Welfare Association. Retrieved from http://www.nicwa.org/resources/catalog/research/2000/02.Child Abuse.pdf

Earle, K. A., \& Cross, A. (2001). Child abuse and neglect: An examination of American Indian data. Seattle, WA: Casey Family Programs. Retrieved from http://www.nicwa.org/research/02.Child_Abuse.pdf

Ehlers, C. L. (2007). Focus on special populations: Variations in ADH and ALDH in Southwest California Indians. Alcohol Research \& Health, 30(1), 14-17.

Eichner, J. E., Cravatt, K., Beebe, L. A., Blevins, K. S., Stoddart, M. L., Fawn, Y., . . . Moore, W. E. (2005). Tobacco use among American Indians in Oklahoma: An epidemiologic view. Public Health Reports, 120(2), 192-199.

Endicott, J., Spitzer, R. L., Fleiss, J. L., \& Cohen, J. (1976). The Global Assessment Scale: A procedure for measuring overall severity of a psychiatric disturbance. Achieves of General Psychiatry, 33(6), 766-771.

Epstein, M., Moreno, R., \& Bacchetti, P. (1997). The underreporting of deaths of American Indian children in California, 1979 through 1993. American Journal of Public Health, 87, 1363-1366.

Evans, E., Spear, S., Huang, Y., \& Hser, Y. (2006). Outcomes of drug and alcohol treatment programs in California. American Journal of Public Health, 96(5), 889896.

Fagan, R. (2006). Counseling and treating adolescents with alcohol and other substance use problems and their families. The Family Journal: Counseling and Therapy for Couples and Families, 14(4), 326-333. 
Falk, D., Hsiao-ye, Y., \& Hiller-Sturmhöfel, S. (2008). An epidemiologic analysis of cooccurring alcohol and drug use and disorders: Findings from the National Epidemiologic Survey of Alcohol and Related Conditions (NESARC). Alcohol Research \& Health, 31(2), 100-110.

Feldstein, S. W., Venner, K. L., \& May, P. A. (2006). American Indian/Alaska Native alcohol-related incarceration and treatment. American Indian \& Alaska Native Mental Health Research: The Journal of the National Center, 13(3), 1-22.

Fiellin, D. A., Carrington, R. M., \& O'Connor, P. G. (2000). Screening for alcohol problems in primary care: A systematic review. Achieves of Internal Medicine, $160,1977-1989$.

Fillmore, K. M. (1984). When angels fall: Women's drinking as cultural preoccupation and as reality. In: S. C. Wilsnack \& L. J. Beckman (Eds.), Alcohol problems in women: Antecedents, consequences, and intervention. New York, NY: Guilford Press.

Fillmore, K. M., Golding, J. M., Graves, K. L., Kniep, S., Leino, E. V., Romelsjo, A., . . Ferrer, H. P. (1998). Alcohol consumption and mortality. III. Studies of female populations. Addiction, 93(2), 219-229.

First, M., Gibbon, M., Spitzer, R. L., \& Williams, J. (1997). Structured clinical interview for DSM-IV axis I disorders, research version, non-patient edition (SCID-I/NP). New York, NY: Biometrics Research, New York State Psychiatric Institute.

Frank, J. W., Moore, R. S., \& Ames, G. M. (2000). Public health then \& now: Historical and cultural roots of drinking problems among American Indians. American Journal of Public Health, 90(3), 344-351. 
French, L. (1995). Adaptations of Aboriginal justice in the United States. Critical Criminology, 6(2), 72-78. doi: 10.1007/bf02585444

French, L. (2004). Alcohol and other drug addictions among Native Americans: The movement toward tribal-centric treatment programs. Alcoholism Treatment Quarterly, 22(1), 81-91.doi:10.1300/J020v22n01_06

First, M. B., Gibbon, M., Spitzer, R. L., Williams, J. B. W., \& Benjamin L. S. (1997). Structured clinical interview for DSM-IV axis II personality disorders (SCID-II). Washington, DC: American Psychiatric Press.

Gold, G. J., \& Nguyen, A. T. (2009). Comparing entering freshmen's perceptions of campus marijuana and alcohol use to reported use. Journal of Drug Education, 39(2), 133-148.

Grandbois, D. G. (2005). Stigma of mental illness among American Indian and Alaska Native nations: Historical and contemporary perspectives. Issues in Mental Health Nursing, 26, 1001-1024.

Grant, B. F., \& Harford, T. C. (1995). Comorbidity between DSM-IV alcohol use disorders and major depression: Results of a national survey. Drug \& Alcohol Dependence, 39, 197-206.

Grant, B. F., Stinson, F. S., Dawson, D. A., Chou, S. P., Dufour, M. C., Compton, W., . . . Kaplan, K. (2004). Prevalence and co-occurrence of substance use disorders and independent mood and anxiety disorders: Results from the National Epidemiologic Survey on Alcohol and Related Conditions. Archives of General Psychiatry, 61(8), 807-816. 
Grim, C. W. (2006). Overview of Indian Health Service and American Indian/Alaska Native research priorities. Washington, D.C.: Indian Health Service. Retrieved from http://www.ihs.gov/PublicInfo/PublicAffairs/Director/2006_Statements/ HHS_Research_Advisory_Council-May_18-web_version.pdf

Grim, C. W. (2007). Tobacco use in Indian country-issues and opportunities. Indian Health Service Tobacco Task Force meeting. Rockville, MD: Indian Health Service. Retrieved from http://www.ihs.gov/publicinfo/publicaffairs/director/2007_Statements/TobaccoUs eInIndianCountry-web.pdf

Guo, J. J., Keck, P. E., Li, H., \& Patel, N. C. (2007). Treatment costs related to bipolar disorder and comorbid conditions among Medicaid patients with bipolar disorder. Psychiatric Services, 58(8), 1073-1078.

Hall, W., Teeson, M., \& Dengenhardt, L. (1999). The 12-month prevalence of substance use disorders in Australian adults: Findings from the National Survey on Mental Health and Well-Being. Addiction, 94, 1541-1550.

Harmon, A. (2010). Indian tribe wins fight to limit research of its DNA. The New York Times. Retrieved from http://www.nytimes.com/2010/04/22/us/22dna.html?_r=1\&hp=\&pagewanted=all

Hasin, D. S., Goodwin, R. D., Stinson, F. S., \& Grant, B. F. (2005). Epidemiology of major depressive disorder: Results from the National Epidemiologic Survey on Alcohol. Achieves of General Psychiatry, 62, 1097-1106. 
Henderson, D. A., Inglesby, T. V., Bartlett, J. G., Ascher, M. S., Eitzen, E., Jahrling, P. B., ... Tonat, K. (1999). Smallpox as a biological weapon. Journal of the American Medical Association, 281, 2127-2137.

Henly, S. J., Struthers, R., Dahlen, B. K., Ide, B., Patchell, B., \& Holtzclaw, B. J. (2006). Research careers for American Indian/Alaska Native nurses: Pathway to elimination of health disparities. American Journal of Public Health, 96(4), 606611. doi: 10.2105/AJPH.2004.054239

Hill, D. L. (2006). Sense of belonging and connectedness, American Indian worldview. Achieves of Psychiatric Nursing, 20(5), 210-216.

Hoff, R. A., \& Rosenheck, R. A. (1999). The cost of treating substance abuse patients with and without comorbid psychiatric disorders. Psychiatric Services, 50, 13091315.

Holkup, P. A., Rodehorst, T. K., Wilhelm, S. L., Kuntz, S. W., Weinert, C., Stepans, M. B. F., ... Hill, W. G. (2009). Negotiating three worlds: Academia, nursing science, and tribal communities. Journal of Transcultural Nursing, 20(2), 164175.

House, L. E., Stiffman, A. R., \& Brown, E. (2006). Unraveling cultural threads: A qualitative study of culture and ethnic identity among urban southwestern American Indian youth parents and elders. Journal of Child \& Family Studies, 15(4), 393-407. doi: 10.1007/s10826-006-9038-9

Howard, B. V., Lee, E. T., Cowan, L. D., Devereux, R. B., Galloway, J. M., \& Go, O. T. (1999). The rising tide of cardiovascular disease in American Indians. The Strong Heart Study. Circulation, 99, 2389-2395. 
Indian Health Service. (1997). Trends in Indian health. Rockville, MD: U.S. Public Health Service. Retrieved from http://www.ihs.gov/publicinfo/publications/trends97/trends97.asp

Indian Health Service. (2007). Indian Health Service: Health Promotion and disease prevention initiative. Retrieved from http://www.ihs.gov/hpdp/documents/IHS_HPDP_Fact_Sheets.pdf

Indian Health Service. (2008a). DHHS: IHS Fiscal year 2009 - Justification of estimates for appropriations committee. Retrieved from http://www.ihs.gov/NonMedicalPrograms/BudgetFormulation/documents/FY\%20 2009\%20IHS\%20Budget\%20CJ\%20Submission.pdf

Indian Health Service. (2008b). IHS fact sheet: Behavioral health. Retrieved from http://info.ihs.gov/Bhealth.asp

Institute of Medicine of the National Academies. (2002). Unequal treatment: Confronting racial and ethnic disparities in health care. Washington, DC: National Academies Press.

Institute of Medicine of the National Academies. (2006). Improving the quality of health care for mental and substance-use conditions: Quality chasm series. Washington, DC: National Academy of Science.

Jane-Llopis, E., \& Matytsina, I. (2006). Mental health and alcohol, drugs and tobacco: A review of the comorbidity between mental disorders and the use of alcohol, tobacco and illicit drugs. Drugs and Alcohol Review, 25, 515-536. 
Jessor, R., Turbin, M. S., \& Costa, F. M. (1998). Protective factors in adolescent health behavior. Journal of Personality \& Social Psychology: Personality Processes and Individual Differences, 75, 788-780.

Kaminer, Y. (2004). Dually diagnosed teens: Challenges for assessment and treatment. Counselor, 5, 62-67.

Kane, G. C., Grever, M. R., Kennedy, J. I., Kuzma, M. A., Saltzman, A. R., Wiernik, P. H., \& Baptista, N. V. (2009). The anticipated physician shortage: Meeting the nation's need for physician services. American Journal of Medicine, 122(12), 1156-1162. doi: 10.1016/j.amjmed.2009.07.010

Kaplan, S. D., Lanier, A. P., Merritt, R. K., \& Siegel, P. Z. (1997). Prevalence of tobacco use among Alaska Natives: A review. American Journal of Preventative Medicine, 26, 460-465.

Katz, M. H. (1999). Multivariable analysis: A practical guide for clinicians. Cambridge, UK: Cambridge University Press.

Kelly, L. C. (1990). Federal Indian policy. New York, NY: Chelsea House. Retrieved from http://www.archives.gov/education/lessons/fed-indian-policy/

Kessler, R. C., Andrews, G., Colpe, L. J., Hiripi, E., Mroczek, D. K., Normand, S.-L. T., . . Zaslavsky, A. (2002). Short screening scales to monitor population prevalences and trends in nonspecific psychological distress. Psychological Medicine, 32, 959-976.

Kessler, R. C., Barker, P. R., Colpe, L. J., Epstein, J. F., Gfroerer, J. C., Hiripi, E., . . . Zaslavsky, A. M. (2003). Screening for serious mental illness in the general population. Archives of General Psychiatry, 60, 184-189. 
Kessler, R. C., Berglund, P., Demler, O., Jin, R., Merikangas, K. R., \& Walters, E. E. (2005). Lifetime prevalence and age-of-onset distributions of DSM-IV disorders in the National Comorbidity Survey Replication. Archives of General Psychiatry, $62,593-768$.

Kessler, R. C., Berglund, P. A., Bruce, M. L., Koch, R., Laska, E. M., Leaf, P. J., \& Wang, P. S. (2001). The prevalence and correlates of untreated serious mental illness. Health Services Research, 36(6 Pt 1), 987-1007.

Kessler, R. C., Berglund, P. A., Glantz, M. D., Koretz, D. S., Merikangas, K. R., Walters, E. E., \& Zaslavsky, A. M. (2002). Chapter 12: Estimating the prevalence and correlates of serious mental illness in community epidemiological surveys. Retrieved on from, http://download.ncadi.samhsa.gov/ken/pdf/SMA013938/MHUS02_Chapter_12.pdf

Kessler, R. C., Berglund, P. A., Walters, E. E., Leaf, P. J., Kouzis, A. C., Bruce, M. L., . . . Schneier, M. (1999). Population based analyses: A methodology for estimating the 12-month prevalence of serious mental illness. In: R. W. Manderscheid, \& M. J. Henderson (Eds.), Mental Health, United States, 1998 (pp.99-109). Washington, DC: U.S. Government Printing Office.

Kessler, R. C., Crum, R. M., Warner, L. A., Nelson, C. B., Schulenberg, J., \& Anthony, J. C. (1997). Lifetime of co-occurrence of DSM-III-R alcohol abuse and dependence with other psychiatric disorders in the National Comorbidity Survey. Achieves of General Psychiatry, 54, 313-321.

King, M., Smith, A., \& Gracey, M. (2009). Indigenous health Part 2: The underlying causes of the health gap. Lancet, 374, 76-85. 
Knight, K., Yankaskas, B. C., Fleg, A., \& Rao, C. (2008). Misclassification of American Indian race in cancer incidence data in North Carolina. Retrieved from http:www.schs.state.nc.us/SCHS

Koren-Karie, N., Sagi-Schwartz, A., \& Joels, T. (2003). Absence of attachment representations (AAR) in adult years: The emergence of a new AAI classification in catastrophically traumatized Holocaust child survivors. Journal of Attachment \& Human Development, 5(4), 381-397.

Krieger, N., Williams, D. R., \& Moss, N. E. (1997). Measuring social class in U.S. public health research: Concepts, methodologies, and guidelines. Annual Review of Public Health, 18(1), 341-378.

Kroenke, K., Spitzer, R. L., \& Williams, J. B. W. (2001). The PHQ-9: Validity of a brief depression severity measure. Journal of General Internal Medicine, 16, 606-613.

LaFromboise, T., Coleman, H., \& Gerton, J. (1999). Psychological impact of biculturalism: Evidence and theory. In K. Keough (Ed.), Social psychology of gender, race, and ethnicity: Readings and projects. New York, NY: McGrawHill.

Lee, R. M., \& Robbins, S. B. (1995). Measuring belongingness: The social connectedness and the social assurance scales. Journal of Counseling Psychology, 4, 232-241.

Lee, R. M. \& Robbins, S. B. (1998).The relationship between social connectedness and anxiety, self-esteem and social identity. Journal of Counseling Psychology, 45, $338-345$.

Lenhardt, A. M. (1997). Grieving disenfranchised loses: Background and strategies for counselors. Journal of Humanistic Education and Development, 35(4), 208-216. 
Limb, G. F., \& Hodge, D. R. (2008). Developing spiritual competency with Native Americans: Promoting wellness through balance and harmony. Families in Society, 89(4), 615-622.

Li-Tzy, W. \& Anthony, J. C. (1999). Tobacco smoking and depressed mood in late childhood and early adolescence. American Journal of Public Health, 89(12), $1837-1840$.

Lomay, V. T., \& Hinkebein, J. H. (2006). Cultural considerations when providing rehabilitation services to American Indians. Rehabilitation Psychology, 51, 36-42.

Lonczak, H. S., Fernandez, A., Austin, L., Marlatt, G. A., \& Donovan, D. M. (2007). Family structure and substance use among American Indian youth: A preliminary study. Family, Systems, \& Health, 25(1), 10-22.

Lowe, J. (2002). Balance and harmony through connectedness: The intentionality of Native American nurses. Holistic Nursing Practice. 16(4), 4-11.

Malcoe, L. H., Duran, B. M., \& Montgomery, J. M. (2004). Socioeconomic disparities in intimate partner violence against Native American women: a cross-sectional study. BMC Medicine, 2, 20-14.

Malcolm, B. P., Hesselbrock, M. N., \& Segal, B. (2006). Multiple substance dependence and course of alcoholism among Alaska Native men and women. Substance Use $\&$ Misuse, 41(5), 729-741.

Manson, S. M. (1989). Long-term care in American Indian communities: Issues for planning and research. The Gerontologist, 29(1), 38-44. 
Manson, S. M. (2000). Mental health services for American Indians and Alaska Natives: Need, use, and barriers to effective care. Canadian Journal of Psychiatry, 45, 617-626.

Manson, S. M., Beals, J., Klein, S. A., \& Croy, C. D. (2005). Social epidemiology of trauma among 2 American Indian reservation populations. American Journal of Public Health, 95(5), 851-859.

Marsiglia, F. F., Nieri, T., \& Stiffman, A. R. (2006). HIV/AIDS protective factors among urban American Indian youth. Journal of Health Care for the Poor and Underserved, 17(4), 1049-2089.

Martikainen, P., Bartley, M., \& Lahelma, E. (2002). Psychosocial determinants of health in social epidemiology. International Journal of Epidemiology, 31, 1091-1093.

May, P. A., \& Gossage, P. (2001). New data on the epidemiology of adult drinking and substance use among American Indians of the northern states: Male and female data on prevalence, patterns, and consequences. American Indian and Alaska Native Mental Health Research, 10(2), 1-26.

May, P. A., \& Moran, J. R. (1995). Prevention of alcohol misuse: A review of health promotion efforts among American Indians. American Journal of Health Promotion, 9, 288-299.

McDermott, P. A., Alterman, A. I., Brown, L., Zaballero, A., Snider, E. C. \& McKay, J. R. (1996). Construct refinement and confirmation of the Addiction Severity Index. Psychological Assessment. 8(2), 182-189.

McDowell, I. (2006). Measuring health: A guide to rating scales and questionnaires. New York, NY: Oxford University Press. 
McKee, S. A., Maciejewski, P. K., Falba, R., \& Mazure, C. M. (2007). Sex differences in the effects of stressful life events on changes in smoking status. Addiction, 98, $847-855$.

McLellan, A. T., Luborsky, L., Cacciola, J., Griffith, J., Evans, F., Barr, H. L., \& O'Brien, C. P. (1985). New data from the Addiction Severity Index reliability and validity in three centers. The Journal of Nervous and Mental Disease, 173(7), 412-423.

McLellan, A. T., \& Marlow-Ferguson, R. (2001). Addiction Severity Index (ASI). Encyclopedia of Drugs, Alcohol, and Addictive Behavior. Retrieved from http://www.encyclopedia.com/doc/1G2-3403100020.html

Miller, N. S., \& Gold, M. S. (1998). Comorbid cigarette and alcohol addiction: Epidemiology and treatment. Journal of Addictive Diseases, 17, 55-66.

Mmari, K., Blum, R., \& Teufel-Shone, N. (2010). What increases risk and protection for delinquent behaviors among American Indian youth? Findings from Three Tribal Communities. Youth \& Society, 4l(3), 382-413.

Mosby. (2009). Mosby's medical dictionary (8th ed.). Philadelphia, PA: Elsevier.

Mulligan, C. J., Hunley, K., Cole, S., \& Long, J.C. (2004). Population genetics, history, and health patterns of Native Americans. Annual Review of Genomics of Human Genetics, 5, 295-315.

Munro, B. H. (2005). Statistical methods for health care research (5th ed.). Philadelphia, PA: Lippincott Williams \& Wilkins. 
Napholtz, L. (2000). Bicultural resynthesis: Tailoring an effectiveness trial for a group of urban American Indian women. American Indian and Alaska Native Mental Health Research. 9(3), 49-70.

National Alliance on Mental Illness (2007). American Indian and Alaska Natives communities mental health facts. Retrieved from http://www.nami.org/ContentManagement/ContentDisplay.cfm?ContentFileID=3 2231

National Institutes of Health. (2005). National survey sharpens picture of major depression among U.S. adults. NIH News. Retrieved from http://www.nih.gov/news/pr/oct2005/niaaa-03a.htm

National Institute of Mental Health. (2010). Closing the gaps: The role of research in reducing mental health disparities in the U.S. Retrieved from http://www.nimh.nih.gov/research-funding/scientific-meetings/2010/closing-thegaps-the-role-of-research-in-reducing-mental-health-disparities-in-the-us.shtml

National League of Nursing. (2009). Race-ethnicity of minority full-time nurse educators by rank. Retrieved from http://www.nln.org/research/slides/pdf/FC0809_F09.pdf National Urban Indian Family Coalition. (2007). Urban Indian American: The status of American Indian \& Alaska Native children and families today. Seattle, WA.

Nemes, S., Jonasson, J. M., Genell, A., \& Steineck, G. (2009). Bias in odds ratios by logistic regression modeling and sample size. Bio Medical Central (BMC) Medical Research Methodology, 9, 56-60.

Neumark-Sztainer, D., Story, M., French, S., Cassuto, N., Jacobs, D. R., \& Resnick, M. D. (1996). Patterns of health compromising behaviors among Minnesota 
adolescents: Sociodemographic variations. American Journal of Public Health, 86(11), 1599-1605.

Owens, P., Myers, M. Elixhauser, A., \& Brach, C. (2007). Care of adults with mental health and substance abuse disorders in U.S. community hospitals, 2004: HCUP factbook no. 10 [AHRQ Publication No. 07-0008]. Rockville, MD: Agency for Healthcare Research \& Quality. Retrieved from http://www.ahrq.gov/data/hcup/factbk10/

Periman, D. (2007). The hidden impact of a criminal conviction: A brief overview of collateral consequences in Alaska. Alaska Justice Forum, 24(3), 6-12.

Polit, D. F., \& Beck, C. T. (2008). Nursing research: Generating and assessing evidence for nursing practice (8th ed.). Philadelphia, PA: Lippincott William \& Wilkins.

Poonwassie, A., \& Charter, A. (2001). An aboriginal worldview of helping: Empowering approaches. Canadian Journal of Counseling, 35(1), 63-73.

Poupart, J., Baker, L., \& Red Horse, J. R. (2009). Research with American Indian communities: The value of authentic partnerships. Children and Youth Services Review, 31(11), 1180-1186. doi: 10.1016/j.childyouth.2009.08.012

Psychosocial. (n.d.). In Merriam-Webster online dictionary. Retrieved May 16, 2009, from http://www.merriam-webster.com/dictionary/psychosocial

Puukka, E., Stehr-Green, P., \& Becker, T. M. (2005). Measuring the health status gap for American Indians/Alaska Natives: Getting closer to the truth. American Journal of Public Health, 95(5), 838-843.

Quercioli, C., Messina, G., Fini, P., Frola, C., \& Nante, N. (2010). Is it possible to evaluate addiction from clinical records? Testing a retrospective addiction 
severity evaluation measure. Substance Use \& Misuse, 45(12), 2045-2058. doi: $10.3109 / 10826081003682826$

Reiger, D. A., Farmer, M. E., Rae, D. S., Locke, B. Z., Keith, S. J., Judd, L. L., \& Goodwin, F. K. (1990). Comorbidity of mental disorders with alcohol and other drug abuse: Results from the Epidemiologic Catchment Area (ECA) Study. Journal of the American Medical Association, 264, 2511-2518.

Rikoon, S. H., Cacciola, J. S., Carise, D., Alterman, A. I, \& McLellan, A. T. (2006). Predicting DSM-IV dependence diagnoses from ASI composite scores. Journal of Substance Abuse Treatment, 3l(1), 17-24.

Rhoades, D. A. (2005). Racial misclassification and disparities in cardiovascular disease among American Indians and Alaska Natives. Circulation, 111, 1250-1256.

Rhoades, D. A., Welty, T. K., Wang, W., Yeh, F., Devereux, R. B., \& Fabsitz, R.R., . . Howard, B. V. (2007). Aging and the prevalence of cardiovascular disease risk factors in older American Indians: The Strong Heart Study. Journal of the American Geriatrics Society, 55(1), 87-94.

Robert Wood Johnson Foundation. (2010). Expanding America's capacity to educate nurses: Diverse state-level partnerships are creating promising models and results. Retrieved from http://www.rwjf.org/files/research/20100608cnf.pdf

Roberts A. R., \& Yeager K. (2004). Mental illness, substance dependence, and suicidality: Secondary data analysis. In: Roberts A.R., \& Yeager K., (Eds.) Evidence based practice manual: Research and outcome measures in health and human services. (pp.70-75), New York, NY: Oxnard. 
Robins, L. N., \& Reiger, D. A. (1991). Psychiatric disorders in America: The epidemiologic catchment area study. New York, NY: The Free Press.

Romanoff, B. D., \& Terenzio, M. (1998). Rituals and the grieving process. Death Studies, 22(8), 697-711.

Ross, H. E. (1995). DSM-III-R alcohol abuse and dependence and psychiatric comorbidity in Ontario: Results from the mental health supplement to the Ontario health survey. Drug and Alcohol Dependence. 39, 111-128.

Rudd, M. D., Berman, A. L., Joiner Jr, T. E., Nock, M. K., Silverman, M. M., Mandrusiak, M., ... Witte, T. (2006). Warning signs for suicide: Theory, research, and clinical applications. Suicide \& Life-Threatening Behavior, 36(3), $255-262$.

Seals, B. F., Burhansstipanov, L., Satter, D.E., Chia, Y. J., \& Gatchell, M. (2006). California American Indian and Alaska Natives tribal groups' care access and utilization of care: Policy implications. Journal of Cancer Education, 2l(S1), S15-S21.

Shern, D. L., Jones, K., Chen, H. J., Jordan, N., Ramoni-Perazzi, J., \& Boothroyd, R. A. (2008). Medicaid managed care and the distribution of societal costs for persons with severe mental illness. American Journal of Psychiatry, 165(2), 254-260.

Shoptaw, S., Rotheram-Fuller, E., Yang, X., Frosch, D., Nahom, D., Jarvik, M.E., . . . Ling, W. (2002). Smoking cessation in methadone maintenance. Addiction, 97, 1317-1328. 
Shore, J., Manson, S. M., \& Buchwald, D. (2002). Screening for alcohol abuse among urban Native Americans in a primary care setting. Psychiatric Services, 53(6), $757-760$.

Silverstein, B. (2002). Gender differences in the prevalence of somatic versus pure depression: A replication. American Journal of Psychiatry, 159(6), 1051-1052.

Sinha, R., \& Rounsaville, B. J. (2002). Sex difference in depressed substance abusers. Journal of Clinical Psychiatry, 63, 616-627.

Smedley, A., \& Smedley, B. (2005). Race as biology is fiction, racism as a social problem is real: Anthropological and historical perspectives on the social construction of race. American Psychologist, 60, 16-26.

Smith, L., Watson, M., Gates, S., Ball, D., \& Foxcroft, D. (2007). Meta-analysis of the association of the Taq1A polymorphism with the risk of alcohol dependency: A huge gene-disease association review. The Journal of Epidemiology, 167(2), 125138. doi: $10.1093 / \mathrm{aje} / \mathrm{kwm} 281$

Smyth, C. L., \& Maclachlan, M. (2004). The context of suicide: An examination of life circumstances thought to be understandable precursors to youth suicide. Journal of Mental Health, 13(1), 83-92.

Sobeck, J. L., Chaplesk, E. E., \& Fisher, C. (2003). Conducting research with American Indians: A case study of motives, methods, and results. Journal of Ethnic \& Cultural Diversity in Social Work, 12(1), 69-84.

Spitzer, R. L., Kroenke, K., Williams, J. B., \& the Patient Health Questionnaire Primary Care Study Group. (1999). Validation and utility of a self-report version of 
PRIME-MD: The PHQ primary care study. Journal of the American Medical Association, 282(18), 1737-1744.

Steele, L. S., Dewa, C. S., Lin, E., \& Lee, K. L. (2007). Education level, income level and mental health services use in Canada: Associations and policy implication. Healthcare Policy, 3(1), 96-106.

Stehr-Green, P., Bettles, J., \& Robertson, L. D. (2002). Effect of racial/ethnic misclassification of American Indians and Alaska Natives on Washington death certificates, 1989-1997. American Journal of Public Health, 92(3), 443-444.

Stewart, M., Makwarimba, E., Reutter, L. I., Veenstra, G., Raphael, D. \& Love, R. (2009). Poverty, sense of belonging and experiences of social isolation. Journal of Poverty, 13(2), 173-195.

Stinson, F. S., Grant, B. F., Dawson, D. A., Ruan, W. J., Huang, B. \& Saha, T. (2006). Comorbidity between $D S M-I V$ alcohol and specific drug use disorders in the United States. Alcohol Research \& Health, 29(2), 94-106. doi:

0.1016/j.drugalcdep.2005.03.009

Stinson, F. S., Ruan, W. J., Pickering, R., \& Grant, B. F. (2006). Cannabis use disorders in the USA: Prevalence, correlates and co-morbidity. Psychological Medicine, $36(10), 1447-60$.

Struthers, R., Kaas, M., Hill, D. L., Hodge, F., DeCora, L., \& Geishirt-Cantrell, B. (2003). Providing culturally appropriate education on type 2 diabetes to rural American Indians: Emotions and racial consciousness. Journal of Rural Community Psychology, E6(1). Retrieved November 2008, from http://www.marshall.edu/jrcp/E6one_struthers.htm 
Struthers, R., \& Lowe, J. (2003). Nursing in the Native American culture and historical trauma. Issues in Mental Health Nursing, 24, 257-272.

Substance Abuse and Mental Health Services Administration. (1993). Final notice establishing definitions for (1) children with serious mental disturbance, and (2) adults with a serious mental illness. Federal Register, 58(96), 29,422-429.

Substance Abuse and Mental Health Services Administration. (1999). Surgeon General's Report: Native American fact sheet. Retrieved from http://mentalhealth.samhsa.gov/cre/fact4.asp

Substance Abuse and Mental Health Services Administration. (2002). A study of the alcohol and drug health of Wisconsin American Indian adults living on or near reservations. Retrieved from http://wch.uhs.wisc.edu/13-Eval/Tools/PDFDocuments/Indian\%20health\%20alcohol\%20WI.pdf

Substance Abuse and Mental Health Services Administration. (2006). Office of Applied Statistics. Treatment episode data set. Retrieved from http://wwwdasis.samhsa.gov/webt/quicklink/US06.htm

Sugarman, J. R., \& Grossman, D. C. (1996). Trauma among American Indians in an urban county. Public Health Reports, 111(4), 321-327.

Szlemko, W. J., Wood, J. W., \& Thurman, P. J. (2006). Native Americans and alcohol: Past, present, and future. Journal of General Psychology, 133(4), 435-451.

Tehee, M., \& Esqueda, C. (2008). American Indian and European American women's perceptions of domestic violence. Journal of Family Violence, 23(1), 25-35.

Thorton, R. (2001). Studying Native America: Problems and prospects. Madison, WI: University of Wisconsin Press. 
Unger, J. B., Soto, C., \& Thomas, N. (2008). Translation of health programs for American Indians in the United States. Evaluation \& Health Professions, 3I(2), $124-144$.

U.S. Bureau of Indian Affairs, Interior. (2009). Indian entities recognized and eligible to receive services from the United States Bureau of Indian Affairs. Federal Register, 74(153), 40218-40223. Retrieved from http://edocket.access.gpo.gov/2009/pdf/E9-19124.pdf

U.S. Bureau of Indian Affairs, Interior (2011). What we do. Retrieved from http://www.bia.gov/WhatWeDo/index.htm

U.S. Census Bureau. (2006). Census 2000. Retrieved from, http://www.census.gov/

U.S. Census Bureau. (2009). Facts for features: American Indian and Alaska Native heritage month. (CB-09 FF.20). Retrieved from http://www.census.gov/newsroom/releases/pdf/cb09-ff20.pdf

U.S. Department of Health and Human Services. (2000). Healthy people 2010:

Understanding and improving health (2nd ed.). Washington, DC: Government Printing Office. Retrieved from http://www.healthypeople.gov/htm

U.S. Department of Health and Human Services. (2001). Culture, race ethnicity: A supplement to mental health; Report of the surgeon general. Retrieved from http://www.surgeongeneral.gov/library/mentalhealth/cre/

U.S. Department of Health and Human Services, Center for Substance Abuse and Treatment. (2005). Substance abuse treatment for persons with co-occurring disorders. Treatment Improvement Protocol (TIP) Series, 42 (DHHS Publication No. [SMA] 05-3992). Rockville, MD: Author. 
U.S. Department of Health and Human Services. (2006a). Healthy people 2010: Focus area 18 mental health and mental disorders. Retrieved from http://www.healthypeople.gov/data/midcourse/html/focusareas/FA18Objectives.h tm

U.S. Department of Health and Human Services, Center for Substance Abuse and Treatment. (2006b). Definitions and terms relating to co-occurring disorders: COCE Overview Paper 1 (DHHS Publication No. [SMA] 06-4163). Retrieved from http://coce.samhsa.gov/cod_resources/PDF/DefinitionsandTerms-OP1.pdf U.S. Department of Health \& Human Services. (2007a). Executive summary and future directions; Health people 2010. Retrieved from http://www.healthypeople.gov/2010/Data/midcourse/html/execsummary/Goal2.ht m

U.S. Department of Health \& Human Services. (2007b). Reauthorization of the Health Care Improvement Act before the committee on Indian affairs U.S. Senate. Retrieved from http://www.hhs.gov/asl/testify/2007/03/t20070308a.html

U.S. Department of Health \& Human Services. (2007c). Findings from 2007; National Survey on Drug Use and Health. Retrieved from http://www.oas.samhsa.gov/nsduh/reports.htm\#2k7.

U.S. Department of Health \& Human Services. (2007d). Depression and the initiation of cigarette, alcohol, and other drug use among young adults. Retrieved from http://www.oas.samhsa.gov/2k7/newusers/depression.htm

U.S. Department of Health \& Human Services. (2009). Healthy People 2020: The road ahead. Retrieved from http://www.healthypeople.gov/hp2020/ 
U.S. Department of Health \& Human Services. (2010a). The Mental Health Parity and Addiction Equity Act. Retrieved from https://www.cms.gov/healthinsreformforconsume/04_thementalhealthparityact.as $\mathrm{p}$

U.S. Department of Health \& Human Services, Substance Abuse \& Mental Health Services Administration. (2010b). Results from the 2009 National Survey on Drug Use and Health: Vol. I Summary of National Findings (HHS Publication No. SMA10-4586 Findings). Rockville, MD. Retrieved from http://oas.samhsa.gov/NSDUH/2k9NSDUH/2k9ResultsP.pdf

U.S. Department of Health and Human Services. (2010c). Can clinicians continue current practice and use the DSM-IV diagnostic criteria. Retrieved from http://questions.cms.hhs.gov/app/answers/detail/a_id/2331

U.S. Department of Health \& Human Services, Substance Abuse and Mental Health Services Administration. (2010d). Office of Applied Statistics. The NSDUH report; substance use among American Indians or Alaska Natives. Retrieved from http://www.oas.samhsa.gov/2k10/182/AmericanIndian.htm

U.S. Department of Labor, Bureau of Labor Statistics. (2011). Labor force statistics from the Current Population Survey. Retrieved from http://www.bls.gov/cps/

Urban Indian Health Commission. (2007). Invisible tribes: Urban Indians and their health in a changing world. Princeton, NJ: Robert Wood Johnson Foundation.

Urban Indian Health Institute. (2008). Reported health and health-influencing behaviors among urban American Indians and Alaska Natives: An analysis of data collected by the Behavioral Risk Factor Surveillance system. Retrieved from 
http://www.uihi.org/wp-content/uploads/2009/01/health_healthinfluencing_behaviors_among_urban_indiansupdate-12102008.pdf

Vernon, I., \& Jumper-Thurman, P. (2005). The changing face of HIV/AIDS among native populations. Journal of Psychoactive Drugs, 37(3), 247-255.

Walters, K. L., Simoni, J. M., \& Evans-Campbell, T. (2002). Substance use among American Indians and Alaska Natives: Incorporating culture in an "Indigenist" stress coping paradigm. Public Health Reports, 140, 189-194.

Wertz, J. S., Cleaveland, B. L., \& Stephens, R. S. (1995). Problems in the application of the Addiction Severity Index (ASI) in rural substance abuse services. Journal of Substance Abuse, 7, 175-188.

Westmoreland, T. M., \& Watson, K. R. (2006). Redeeming hollow promises: The case for mandatory spending on health care for American Indians and Alaska Natives. American Journal of Public Health, 96(4), 600-605.

Whitbeck, L. B., Adams, G. W., Hoyt, D. R., \& Chen, X. (2004). Conceptualizing and measuring historical trauma among American Indian people. American Journal of Community Psychology, 33(3/4), 119-130.

Whitbeck, L. B., Hoyt, D. R, Johnson, K., \& Xiaojin, C. (2006). Mental disorders among parents/caretakers of American Indian early adolescents in the Northern Midwest. Social Psychiatry \& Psychiatric Epidemiology, 41(8), 632-640.

Whitbeck, L. B., Johnson, K. D., Hoyt, D. R., \& Walls, M. (2006). Prevalence and comorbidity of mental disorders among American Indian children in the northern Midwest. The Journal of Adolescent Health: Official publication of the Society for Adolescent Medicine, 39(3), 427-434. 
Whitesell, N. R., Beals, J., Mitchell, C. M., Novins, D. K., Spicer, P., O'Connell, J., \& Manson, S. M. (2007). Marijuana initiation in 2 American Indian reservation communities: Comparison with a national sample. American Journal of Public Health, 97(7), 1311-1318.

Wilkinson, R. G., \& Pickett, K. E. (2006). Income inequality and population health. Social Science \& Medicine, 62(7), 1768-1784.

Winkler, A. M. (1968). Drinking on the American frontier. Quarterly Journal of Studies on Alcohol, 29, 413-445.

Witte, T. K., Timmons, K. A., Smith, A. R., \& Joiner, T. E. (2009). Do major depressive disorder and dysthymic disorder confer differential risk for suicide? Journal of Affective Disorders, 115(1-2), 69-78.

World Health Organization. (2004). Chapter 4: Determinants (risk and protective factors) indicators. Retrieved from http://www.who.int/hiv/pub/me/en/me_prev_ch4.pdf

World Health Organization. (2007). International statistical classification of diseases and related health problems [ICD-10] (10th revision). Retrieved from http://www.who.int/classifications/icd/en/

Yellow Horse Brave Heart, M. (1999). Gender differences in the historical trauma response among the Lakota. Journal of Health \& Social Policy, 10(4), 1-21.

Yellow Horse Brave Heart, M., \& DeBruyn, L. (1998). The American Indian Holocaust: Healing historical unresolved grief. National Center for American Indian \& Alaska Native Mental Health Research, 8(2), 56-78. 
Young, R. S., Joe, J. R., Hassin, J., \& St. Clair, D. (2001). Addressing psychological issues and problems of co-morbidity for Native American clients with substance abuse problems: A conference proceedings. American Indian and Alaska Native Research: The Journal of the National Center, 10(2), 5-113. Retrieved from http://www.ucdenver.edu/academics/colleges/PublicHealth/research/centers/CAI ANH/journal/Documents/Volume\%2010/10(2)_1_Young_Editorial.pdf

York, J. L., \& Welte, J. W. (1994). Gender comparisons of alcohol consumption in alcoholic and nonalcoholic populations. Journal of Studies on Alcohol, 55, $743-$ 750.

Zanis, D. A., McLellan, T. A, \& Corse, S. (1997). Is the Addiction Severity Index a reliable and valid instrument among clients with severe persistent mental illness and substance abuse disorders? Community Mental Health Journal, 33(3), 213227. 
Appendix B

Department of Health and Human Services IHS NIRB Approval

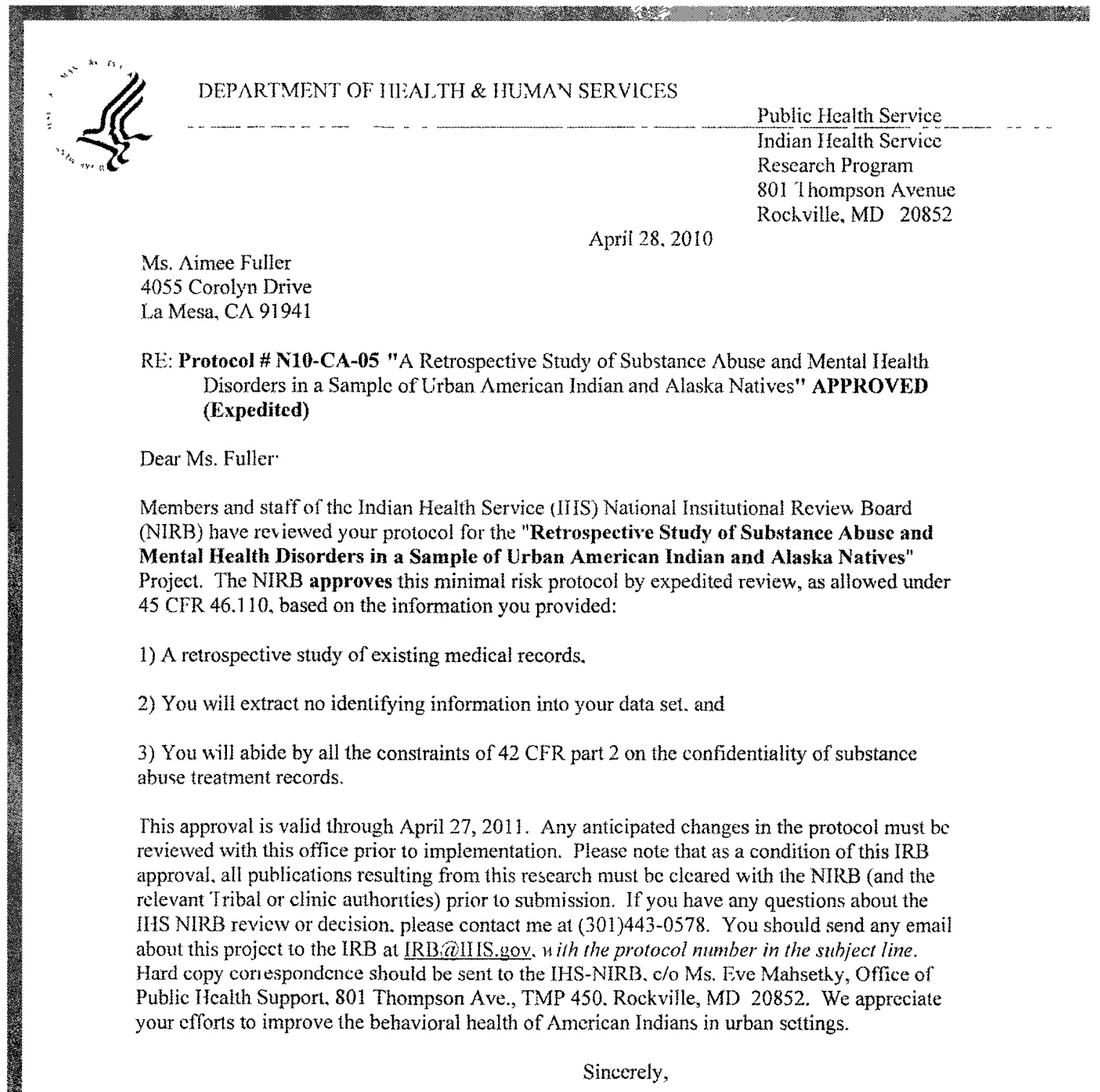

Alan Trachtenberg, MD. MPH.

IJuman Research Protections Administrator IRB00000646: FWA00008894 